\title{
On Control of the Excitable Dynamics in the Heart
}

\author{
Dissertation \\ for the award of the degree \\ "Doctor rerum naturalium" \\ of the Georg-August-Universität Göttingen \\ within the doctoral program Physics of Biological and Complex Systems \\ of the Göttingen Graduate School for Neurosciences, Biophysics, and Molecular \\ Biosciences (GGNB)
}

submitted by Henrik tom Wörden

from Stade

Göttingen, 2018 


\section{Thesis Committee}

Prof. Dr. Stefan Luther, Biomedizinische Physik, Max-Planck-Institut für Dynamik und Selbstorganisation

Prof. Dr. Jörg Enderlein, Drittes Physikalisches Institut, Georg-August-Universität Göttingen

Dr. Marco Tarantola, Dept. Fluid Dynamics, Pattern Formation, and Nanobiocomplexity, Max-Planck-Institut für Dynamik und Selbstorganisation

\section{Members of the Examination Board}

Referee: Prof. Dr. Stefan Luther, Biomedizinische Physik, Max-Planck-Institut für Dynamik und Selbstorganisation

$2^{\text {nd }}$ Referee: Prof. Dr. Jörg Enderlein, Drittes Physikalisches Institut, Georg-August-Universität Göttingen

Further members of the Examination Board:

Prof. Dr. Jens Frahm, Biomedizinische NMR, Max-Planck-Institut für Biophysikalische Chemie

Prof. Dr. Stefan Klumpp, Institut für Nichtlineare Dynamik, Georg-August-Universität Göttingen

Prof. Dr. Wolfram Kollatschny, Institut für Astrophysik, Georg-August-Universität Göttingen

Prof. Dr. Florentin Wörgötter, Drittes Physikalisches Institut, Georg-August-Universität Göttingen

Date of oral examination: 14 Dez 2018 
dedicated

to my great love

Kathrin 


\section{Contents}

1 Introduction 1

1.1 Excitable Media . . . . . . . . . . . . . . . . . . . . 1

1.2 The Heart . . . . . . . . . . . . . . . . . . . . . . . . 3

1.3 Ventricular Fibrillation . . . . . . . . . . . . . . . . 3

1.4 Effect of Electric Shocks on the Membrane Voltage . . . . . . . . . . 6

1.5 New Defibrillation Approaches . . . . . . . . . . . . 7

1.6 Synchronization of the Electric Activity in Cardiac Tissue . . . . . . 8

1.7 Scope of the Thesis . . . . . . . . . . . . . . . . . . 9

2 Simulations $\quad \mathbf{1 1}$

Simultaneous Unpinning of Spiral Waves in 2D Excitable Media . . . . . . 13

3 Experiments $\quad \mathbf{2 3}$

3.1 Langendorff Perfusion System . . . . . . . . . . . . . . . . . . . . 23

3.2 Optical Mapping . . . . . . . . . . . . . . . . . . . . . 24

3.3 Electric Stimulation . . . . . . . . . . . . . . . . . . 25

3.4 Data Acquisition . . . . . . . . . . . . . . . . . 26

3.5 Animal Model . . . . . . . . . . . . . . . . . . . . 26

3.6 Experimental Procedure . . . . . . . . . . . . . . . . . . . 27

3.7 Data Management . . . . . . . . . . . . . . . . . . . . . 28

3.8 Preprocessing of Optical Mapping Video Data . . . . . . . . . . . . 30

4 Results 33

4.1 Control by Periodic Pulse Sequences . . . . . . . . . . . . . . 33

4.1.1 Termination of Ventricular Fibrillation with Periodic Pulses . . 35

4.1.2 Area Activated by Different Pulsing Frequencies and Pulse

Strength . . . . . . . . . . . . . . . 38

4.1.3 Characterization of Ventricular Fibrillation . . . . . . . . . 40

4.1.4 The Role of the Dominant Frequency . . . . . . . . . . . . . . 42

4.1.5 Synchronization of Ventricular Fibrillation . . . . . . . . . . . 44

4.1.6 Successive Synchronization during Periodic Pulses . . . . . . . 48

4.1 .7 Section Summary . . . . . . . . . . . . . . . . . 54

4.2 Synchronization Preceding Termination of VF . . . . . . . . . . . 55

4.2 .1 Dose Response Curve . . . . . . . . . . . . . . . 55

4.2 .2 Syncrolation . . . . . . . . . . . . . . . . . . 59

4.2 .3 Single Shock . . . . . . . . . . . . . . . . . . . 62

4.2.4 Five Biphasic Underdrive Pulses . . . . . . . . . . . . . . . 62 
4.2.5 Comparison of Characteristic Energies . . . . . . . . . . . 63

4.2.6 Influence of the Period Factor . . . . . . . . . . . . . . 67

4.2 .7 Influence of the Delay . . . . . . . . . . . . . . . . . . 70

4.2.8 Synchronization as a Predictor for Termination . . . . . . . . 72

4.2 .9 Section Summary . . . . . . . . . . . . . . . . . . . . . . 74

4.3 Mechanisms of Defibrillation . . . . . . . . . . . . . 75

4.3.1 Simultaneous Activation . . . . . . . . . . . . . . 76

4.3.2 Homogeneous activation . . . . . . . . . . . . . 78

4.3 .3 Section Summary . . . . . . . . . . . . . . . . . . 82

5 Discussion $\quad \mathbf{8 3}$

5.1 Simultaneous Unpinning . . . . . . . . . . . . . . . . . . 83

5.2 Syncrolation . . . . . . . . . . . . . . . . 83

5.3 Synchronization . . . . . . . . . . . . . . . . 85

5.4 Termination through Synchronization . . . . . . . . . . . 86

5.4.1 The Role of the Dominant Frequency . . . . . . . . . . . 87

5.4 .2 Multisite Pacing . . . . . . . . . . . . . . . . . 88

5.5 Mechanisms of Defibrillation . . . . . . . . . . . . . . . 88

$\begin{array}{llr}6 & \text { Summary } & 91\end{array}$

$\begin{array}{ll}\text { A Appendix } & 101\end{array}$

A.1 Abbreviations . . . . . . . . . . . . . . . . . . . . . 101

A.2 Acknowledgement. . . . . . . . . . . . . . . . . . . . . . 101

A.3 Competing Interests . . . . . . . . . . . . . . . . 102

A.4 Curriculum Vitae . . . . . . . . . . . . . . . . . . . . 102

A.5 Figures . . . . . . . . . . . . . . . . . 102

A.6 Tables . . . . . . . . . . . . . . . . . . . . . 108

A.7 Supplementary Material to Manuscript . . . . . . . . . . . . . . . . . . 114 



\section{Introduction}

Already the frequent use of the heart in sayings or lyrics illustrates the central role of the heart as the driver of our circulatory system. It literally keeps us alive. On the contrary, problems with the heart are often dramatic: Diseases of the circulatory system in general were the most frequent cause of death in 2015 in Germany [15]. The main motivation for the work at hand is a lethal heart condition called ventricular fibrillation (VF). VF is an arrhythmia where the heart contracts in an uncoordinated manner such that blood transport is essentially stopped and immediate treatment is crucial. Intense research aims at better understanding this heart condition and at providing new treatments, as the current clinically used method to terminate VF has severe drawbacks. The many different facets of VF are the reason that this research has a very interdisciplinary nature. For example, the tools that I learned as a physicist are especially helpful when studying the complex dynamics of electric activity that persist in the heart muscle during VF. These dynamics will be the main object of investigation in this thesis. They share some qualitative features with the dynamics of the large group of excitable media. In the following, I will therefore introduce excitable media and describe how they help us in understanding the electric dynamics of the heart, before afterwards describing the heart and its arrhythmias in more detail.

\subsection{Excitable Media}

Already Mines [60] described in 1914 that a stimulation of a strip of heart tissue by pricking it with a needle can cause the tissue to go into an excited state and that this state spreads over the tissue. He further observed that another excitation can only be caused after some time has passed since the previous one. These are features of an excitable medium.

In an excitable system, a small perturbation from the steady state decays while a stimulation above some threshold will create a self-amplified response, an excitation, that prevents further excitations for some time, called the refractory period. This behavior is illustrated in fig. 1.1, where two exemplary trajectories of the Barkley Model [6], a simple system of two coupled ordinary differential equations, are shown in phase space. The dots that lie on the trajectories in fig. 1.1 are equally spaced in time in order to provide a better impression of the dynamics. Here, high values of the variable $u$ correspond to the excited state and high values of the variable $v$ mean that the system is refractory. The blue trajectory depicts a subthreshold stimulation to the point $A$, that decays, i.e. the trajectory leads back to the steady state at the origin. However, the orange trajectory starts from a superthreshold stimulation at point B. 


\section{Introduction}

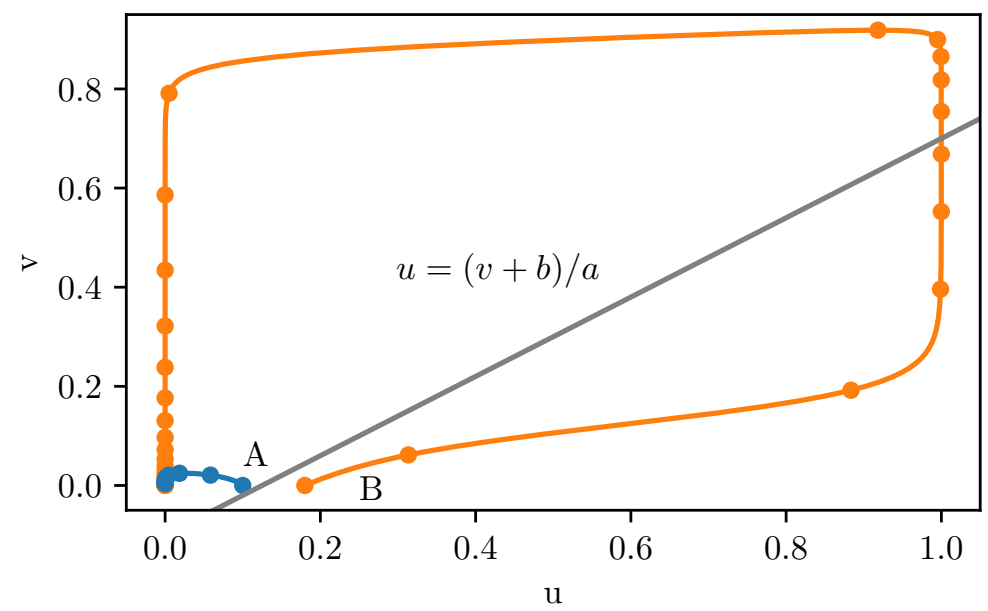

Figure 1.1: Two trajectories of the Barkley model are shown. The blue trajectory starting at A illustrates that a subthreshold stimulation decays. The orange trajectory starting at B illustrates that a superthreshold stimulation leads to a self-amplified response and a large excursion through the phase space. The line $u=(v+b) / a$ shown in gray is a nullcline of the system that needs to be crossed by a stimulation for a self-amplified response.

This perturbation is amplified by the system: The value of the $u$ variable increases further and the trajectory does a large detour through the phase space. Here, we can identify why $u$ is typically called the fast and $v$ the slow variable: While changes of the $u$ variable occur quickly (wide spacing of dots on the trajectory), changes of the $v$ variable are slower. The local dynamics of the Barkley model are defined by the following ordinary differential equations:

$$
\begin{gathered}
\frac{\partial u}{\partial t}=\frac{1}{\varepsilon} u(1-u)\left(u-\frac{v+b}{a}\right) \\
\frac{\partial v}{\partial t}=u-v .
\end{gathered}
$$

$a, b$ and $\varepsilon$ are parameters of the model. These equations show that for a self-amplified response a stimulus needs to increase $u$ until it crosses the nullcline $u=(v+b) / a$ (see fig. 1.1). The Barkley model is used in my numerical investigations presented in chapter 2. Please refer to e.g. [7] for more information on the Barkley model.

Not surprisingly, a medium that locally shows the properties of an excitable system and that allows excitation to spread is called an excitable medium. The dynamics of excitable media can show a variety of excitation patterns such as traveling waves or spiral waves, but also turbulent dynamics [5]. Examples of excitable media are aggregations of Dictyostelium discoideum amoebae [72], the Belousov-Zhabotinsky reaction [86] or the cardiac muscle. 


\subsection{The Heart}

We just learned that the heart is an excitable medium. In order to allow precise descriptions in later sections of this thesis, let us briefly recapitulate the aspects that will be important in the following.

The heart is divided in two atria and two ventricles (see fig. 1.2). Blood with low oxygen concentration enters the right atrium and is then pushed into the right ventricle (RV). From there, blood is pumped for oxygenation into the lungs before it flows in the left atrium and then in the left ventricle (LV) from where the blood is distributed to the whole body.

The heart tissue mainly consists of cardiomyocytes that have at rest a specific transmembrane voltage (around $-90 \mathrm{mV}$ in the $\mathrm{LV}$ ) due to different ion concentrations inside and outside of the cell. A sufficient depolarization of this transmembrane voltage leads to a series of ion exchanges of a cell with its surrounding medium that leads to an even larger change of the transmembrane voltage (to around $50 \mathrm{mV}$ in the LV), i.e. an excitation. This excitation leads to a contraction of the cell. Myocytes are coupled to neighboring cells with so called gap junctions such that the depolarization can spread through the tissue.

The normal heart beat, also known as normal sinus rhythm (NSR), is controlled by the sinoatrial node (1 in fig. 1.2) that consists of special heart cells that are periodically self-excited. This excitation spreads over the atria and leads to their contraction, is delayed in the atrioventricular node and is then propagated quickly through the His bundles and Purkinje fibers (see fig. 1.2) into the ventricles such that they are excited nearly simultaneously which allows a coordinated contraction of the ventricles and thus an efficient blood transport.

\subsection{Ventricular Fibrillation}

The condition, when the uniform activation of the heart muscle during NSR is interrupted and the activation becomes uncoordinated, is called ventricular fibrillation (VF) and was briefly introduced in the beginning of the chapter 1. The electric dynamics in the heart tissue form complex, irregular patterns comprising small wavelets and spiral waves which can be visualized with optical mapping (see fig. 1.3; optical mapping will be described in section 3.2) or using an electrocardiogram (ECG). Figure 1.4 shows an ECG where a NSR first changes into a faster ventricular tachycardia (VT) and then further deteriorates into VF. These complex activation patterns indicate that the heart muscle is contracting uncoordinatedly during VF and thus blood transport is essentially prevented. Therefore, an immediate treatment is crucial.

Despite over a century of research, since experiments of Mayor [57] and Mines [60] (see $[43,68,42]$ for (historical) reviews), it is still disputed how VF is maintained in the heart. One concept attributes an important role to so called rotors, centers of 


\section{Introduction}

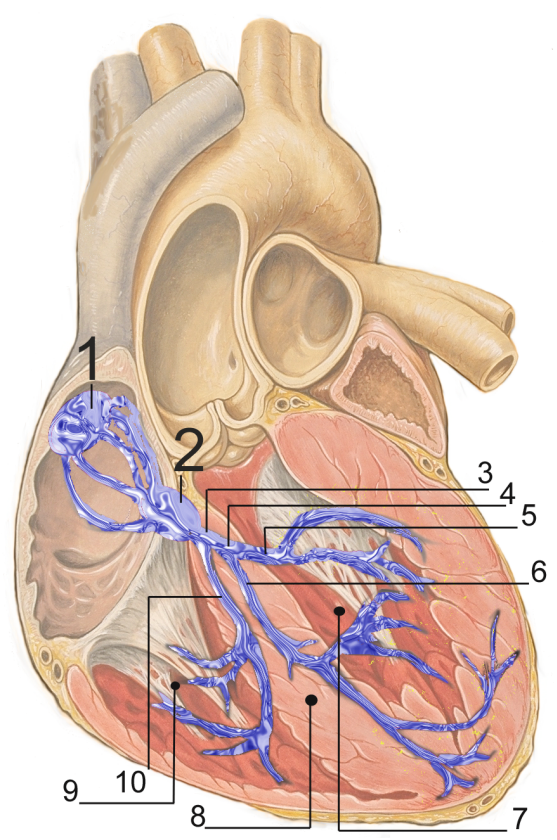

Figure 1.2: Electrical conduction system of the heart. 1 Sinoatrial node; 2 Atrioventricular node; 3 Bundle of His; 4 Left bundle branch; 5 Left posterior fascicle; 6 Left-anterior fascicle; 7 LV; 8 Ventricular septum; 9 RV; 10 Right bundle branch. Illustration from [36]

rotating activity that can be imagined as the cores of spiral waves. These centers create waves that propagate to other regions of the heart and possibly break up in the process and thus form the complex dynamics that we observe [65]. An alternative theory states that VF is maintained by the many small waves itself that propagate in a random fashion over the heart muscle and no driver as e.g. a rotor is necessary [42].

The only method for terminating VF that is used clinically is the application of a high energy electric shock. This shock excites the whole tissue and due to the refractory period, this means that for some time after this excitation no activity can be caused or propagate and thus the VF is terminated (see the early work published in [25] and [50] for details on the effect of a defibrillation shock). The NSR can then start again.

According to the critical mass hypothesis, an electric shock that depolarizes a critical amount of myocardium leads to the termination of VF because not enough tissue remains for fibrillatory waves to propagate into. This hypothesis is supported by experimental findings of Zipes et al. [91] from 1975. This work fueled simulation studies that investigated the depolarisation of defibrillation techniques and then conclude that termination of VF would occur if e.g. $95 \%$ of the myocardium was excited [2].

Defibrillators are devices for the termination of VF that can be connected to a person suffering from VF via electrodes, allow to apply an electric shock in an ambulant 

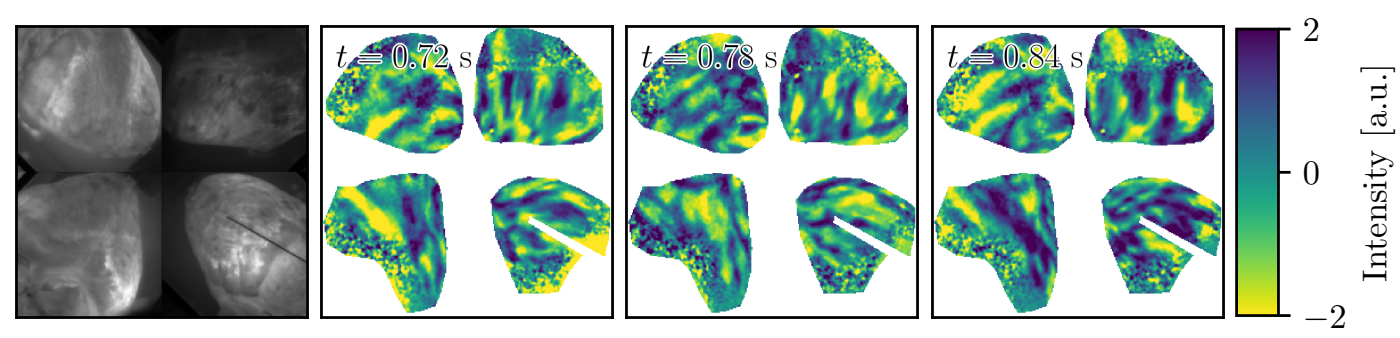

Figure 1.3: The complex patterns of electric activity on the cardiac surface during VF are shown. The data stems from an optical mapping recording using four cameras (see chapter 3 for details; experiment of 3 May 2018; recording at 19:36:59). On the left an unfiltered frame of the optical mapping recording is shown in a gray scale where the four perspectives of the cameras can be identified. The three colored images next to it are processed frames (see section 3.8) at times indicated in the upper left corner. These images illustrate that the electric dynamics during VF are very complex and can consist of many small waves.

setting and can be operated by untrained people. However, there are also implantable cardioverter-defibrillators (ICDs), that automatically detect the presence of a VF and apply the electric shock to terminate it.

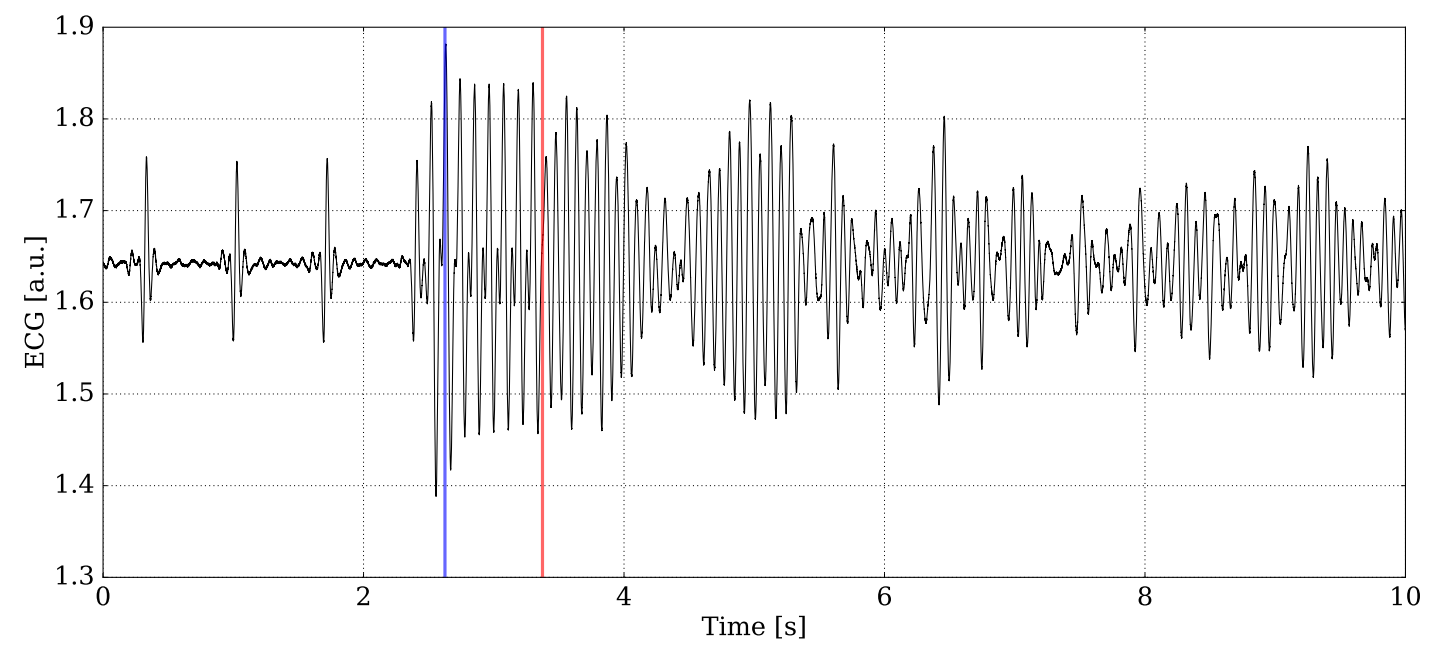

Figure 1.4: An ECG time series from an ex-vivo experiment with a rabbit heart showing the transition of a sinus rhythm to VT and VF is depicted. The blue line marks the onset of VT with an approximate frequency of $8.75 \mathrm{~Hz}$. The red line marks the beginning of the deterioration into VF at a dominant frequency of $12.5 \mathrm{~Hz}$. This ECG has been recorded on 1 Feb 2017 by Sebastian Berg and Edda Boccia. The figure is from [73] by courtesy of A. Schlemmer. 


\section{Introduction}

There are however some drawbacks to the above described single shock defibrillation: When an ICD detects the arrhythmia, the patient usually still is conscious and the application of the shock is traumatically painful $[83,3]$. There is also evidence, that the high energy of electric shocks causes damage in the heart tissue making future heart diseases more likely [74, 47]. An international research effort thus aims at better understanding VF and providing alternatives to high-energy single shock defibrillation.

Generally very diverse approaches are followed to provide alternative defibrillation techniques, such as optogenetical approaches or new drugs. There are however also approaches that intend to use the same tool as conventional single shock defibrillation, i.e. electric field pulses, in a new, advantageous way. Before describing these new techniques, I will describe in the following section how their means, electric far field pulses, affect the heart muscle.

\subsection{Effect of Electric Shocks on the Membrane Voltage}

We know that the application of single high-energy electric far field shock can terminate VF by exciting the whole heart tissue at once. How does this work?

Both, the cardiomyocytes and the extra cellular medium, are filled with a conducting fluid and neighboring myocytes are electrically coupled via gap junctions [21]. Thus the high resistive cell membrane divides two conductive domains. The application of an electric field thus leads to an electric current through both domains, the intraand extra cellular spaces. However, this requires a transmembrane current flow near tissue boundaries which in turn leads to a hyper- or depolarization of the membrane depending on the polarity of the electric field [66]. The effect of an electric field near heterogeneities is thus similar to the effect of a local current injection with an electrode. The hyper- and depolarization caused by electric fields are therefore also called virtual electrodes. An electric field of sufficient strength can in this way cause a depolarization that leads to an excitation of the respective cardiac cells and thus potentially create a propagating excitation wave (see fig. 1.5). Various kinds of tissue inhomogeneities, such as changes of fiber direction or boundaries to unexcitable or non-conductive tissue can lead to the creation of virtual electrodes [84, 67, 79].

Furthermore, the size and shape of boundaries determine the strength of a corresponding depolarization $[12,67]$. The larger the field strength is the smaller the heterogeneities can be to reach a sufficient depolarization for an excitation. Thus, (it can be assumed that) single shock defibrillation has a sufficient field strength that it leads to an excitation of the whole tissue, as it was stated by Connolly et al. [22].

Luther et al. [55] showed that the radii distribution of the vasculature in the heart muscle follows a power law and thus the distribution of wave sources can be estimated for certain field strengths. The information where in the heart muscle electric pulses can directly control the dynamics of the tissue contributed to the design of a new defibrillation technique described in the following section. 
c)

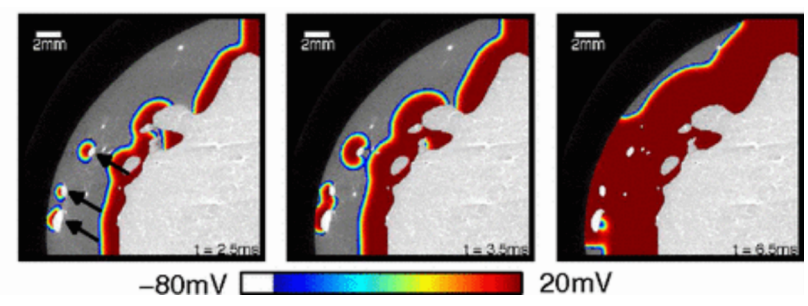

Figure 1.5: The excitation of cardiac tissue in response to an electric field stimulation is shown in a two-dimensional cross section of the LV of a rabbit. The image data shown in a gray scale stems from a micro-CT and defines the shape of the tissue domain used in a computer simulation that models the excitation of the tissue and the propagation of waves. Please note, how the excitation starts at protrusions and heterogeneities in the tissue. These images are the third row of figure 4 in [12]. Please refer to this publication for further details.

\subsection{New Defibrillation Approaches}

Due to the relation described in the previous section, electric far field pulses that have a smaller energy than the one required for single shock termination do not excite the heart tissue everywhere. The smaller the field strength of the pulse is the larger the heterogeneities need to be for an excitation and the fewer the locations are where control is exercised.

Still, in [29] the authors present a technique that terminates atrial fibrillation (AF) with multiple electric far field pulses. Additionally, Luther et al. [55] showed that the use of this low-energy antifibrillation pacing (LEAP) allows an $84 \%$ reduction in-vivo and $91 \%$ reduction in-vitro in energy per pulse needed to terminate AF compared to a single (high-energy) shock. They also reported an $85 \%$ energy reduction per pulse compared to a single shock when terminating VF in canine wedge preparation.

The authors state that the field pulses directly interact with the cores of the activation during VF and that the tissue is successively synchronized. They describe that the pulses at some point cause a simultaneous activation of the whole tissue. Thus, their method essentially achieves with multiple low-energy pulses a similar effect as a single high-energy shock (see also [33]).

Another technique that employs multiple far field pulses is the multistage electrotherapy to terminate AF published by $\mathrm{Li}$ et al. [53]. The technique that has three stages where in each stage a different pulse series is applied, two of which use far field pulses and the last a series local stimulations. The mechanistic understanding that the authors present differs however from what was described above: They attribute an important role to the stabilization of (spiral) waves by heterogeneities in the tissue which might maintain AF. Li et al. [53] thus state that the three stages "mechanistically relate to the (1) unpinning of wavefronts that maintain AF, (2) prevention of re-repinning [sic] 


\section{Introduction}

of wavefronts to tissue heterogeneities such as scar, and (3) annihilation of remaining wavefronts."

The two aforementioned techniques might be motivated by a different mechanistic understanding of the maintenance and termination of fibrillation (here AF and VF), but the main concept, a localized exercise of control due to the use of comparably low field strength, is the same.

\subsection{Synchronization of the Electric Activity in Cardiac Tissue}

As mentioned in the last section, in [55] and also in the more recent publication [44], it was postulated that a synchronization of the electric activity in the cardiac tissue facilitates defibrillation. In the following, I will provide an overview of previous work that covered the synchronization of cardiac tissue.

Dillon [25] described how both the depolarization and the repolarization of the myocardium is synchronized by a single high-energy defibrillation shock. Wilson and Moehlis [85] presented a mathematical framework to investigate the optimal pulse shape for synchronization of the myocardium. They however exclusively consider pulse sequences that are applied within one cycle length of the cardiac activity. The kind of synchronization that is studied in [85] and [25] thus differs significantly from the synchronization with multiple pulses over a longer time as in the work by Luther et al. [55].

Pak et al. [62] investigated the synchronization of cardiac electric dynamics during VF caused by electric stimulation with four local electrodes using a feedback loop coupled to the optical signal. They show that the standard deviation of the cycle length of activation is reduced in the tissue near the electrodes during the pacing. Also, the number of phase singularities is significantly reduced in the region surrounded by the four electrodes. A feedback mode where all four electrodes are commonly controlled according to the signal of a reference location has a success rate in terminating VF of $\approx 13 \%$ which is the highest success rate that they observed for different feedback modes.

Pak et al. [63] also published a follow up study where they used the above synchronization with four local electrodes before the application of a specifically timed high-energy single shock. They showed, that the energy for $50 \%$ success rate of the single shock was reduced by about $10 \%$ compared to the defibrillation without preceding synchronization.

In related work, Nanthakumar et al. [61] reported on the control of electric dynamics in the LV during VF using two lines of electrodes and Meunier et al. [59] used a grid of electrodes to synchronize the activity in a large region of the epicardial surface.

In numerical investigations of multi-site pacing with four electrodes and a feedback control, Hosfeld et al. [40] find that they can remove complex activation patterns in 
up to $45 \%$ of the cases and that the probability to achieve this depends on the cycle length of the VF.

The above enumeration of previous studies shows that literature exists on the synchronizing effect of a single high-energy electric far field pulse and on the control using multi-site pacing techniques. I am however not aware of any publication that considers in detail the synchronization process caused by multiple, periodic, electric far field pulses delivered over multiple cycle length of the cardiac dynamics.

\subsection{Scope of the Thesis}

In section 1.5, I described the technique for terminating $\mathrm{AF}$ by $\mathrm{Li}$ et al. [53] that specifically targets pinned wavefronts, i.e. wavefronts that are attached to and stabilized by a heterogeneity in the medium. In literature, the concept of pinned spiral waves is frequently associated with $\mathrm{VF}$ and constraints for its termination [39, 10, 87, 64 , $23,34]$. Therefore, the behavior of a single pinned spiral wave has been extensively studied [70, 8, 64, 41]. However, in case pinned spirals do play a role in VF and its termination, then it is not clear why only a single pinned spiral wave should exist in the heterogeneous heart muscle. In spite of this, I am not aware of literature describing the removal of multiple pinned spirals except for [39]. Thus, I studied the case of multiple pinned spiral waves using numerical simulations of a generic, simple model of an excitable medium. This work will be described in the manuscript contained in chapter 2. Additionally

The results of this theoretical and numerical work motivated the design of a new defibrillation technique which I investigated experimentally. This technique and the experimental results will be described in section 4.2. This new technique employs the synchronization of cardiac activity with multiple electric far field pulses of the tissue and has thus per se some similarities with the approach described in [55].

However, as the overview of previous work on synchronization in the previous section showed, the mechanism of synchronization of cardiac activity by electric far field shocks is so far not understood well enough to answer the following questions:

- What are the properties of pulse sequences that cause the strongest synchronization?

- How does the synchronization depend on the dynamics in the heart?

- How does the synchronization develop over time during the application of a series of periodic pulses?

I conducted whole heart perfusion experiments with rabbit and pig hearts in order to investigate the novel defibrillation method on the one hand and to answer the above mentioned questions on the other hand. In these experiments, I used state of the art panoramic optical mapping with high speed cameras and simultaneous electrophysiological measurements. These measurements provided detailed and direct information on the cardiac electric dynamics with a high temporal and spatial resolution. In 


\section{Introduction}

my work, I contributed to the development of the custom-built software that allows the application of various electric stimuli, also with near real-time feedback. These capabilities are essential when studying how the response of cardiac tissue to stimuli depends on the cardiac dynamics.

The extensive data that was recorded in those experiments also allowed the quantitative analysis of many aspects of the synchronization process. In particular, I investigated how the control of periodic pulses does not only depend on their voltage but also on the ratio of the pacing frequency and the frequency of the cardiac excitation. This includes not only the degree of synchronization that is reached at the end of long pulse series but also temporal development.

My work can thus be grouped into three major topics. First, the theoretical and numerical work on the unpinning of multiple spirals in a generic two-dimensional excitable media will be described in chapter 2. This work inspired the design of a novel defibrillation technique that employs synchronization with far field pulses. Thus, the two other parts are the investigation of this novel technique on the one hand (section 4.2) and the fundamental research on control of the cardiac electric activity with electric far field pulses on the other hand (section 4.1). The two latter parts are based on experimental work which will be describe in chapter 3 . 


\section{Simulations}

In the context of this thesis, the main advantage of studying simple excitable media, like a homogeneous medium with the local dynamics of the Barkley model (see section 1.1), is that these models are on the one hand much simpler to handle and understand but on the other hand still share important features with the heart. Sometimes, it is therefore possible to transfer findings from those simple models to the heart. For example, a spiral wave in a homogeneous excitable medium can be controlled with a wave train created from some local activation when it has a larger frequency than the rotation frequency of the spiral (see e.g. [90]). The wave train pushes the spiral through the medium until the core collides with a system boundary and the spiral vanishes. This is a plausible explanation why anti-tachycardia pacing, a periodic, local stimulation of cardiac tissue via an electrode, is often able to terminate slow $\mathrm{VT}^{1}$, which is another dangerous cardiac arrhythmia that may precede VF (see also fig. 1.4) and its activity is often associated with a spiral wave.
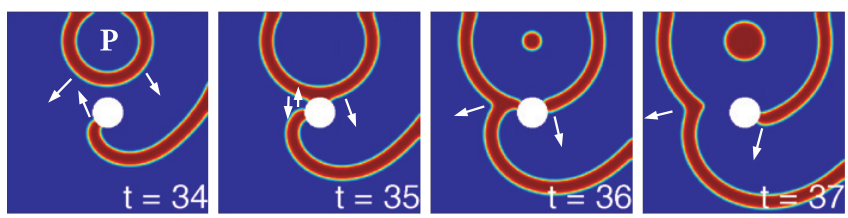

Figure 2.1: The figure illustrates how an obstacle in a simple, two-dimensional, homogeneous, excitable medium can prevent the removal of the spiral by a periodic wave train. At point $\mathrm{P}$ a local periodic stimulus is applied that causes a train of circular wave fronts. The spiral wave that is anchored at the heterogeneity (white circle) however is not removed. The wave propagation illustrated by white arrows leads to annihilation of wave fronts such that always one wave front remains attached to the obstacle. The shown graphics is part a) of Figure 1 from [11].

As already mentioned, spiral waves can pin to obstacles in the excitable medium. This pinning stabilizes the spiral wave and prevents for example a drift of the spiral or that the spiral is driven out of the medium by wave trains as described above (see fig. 2.1 and [11]). Extensive research considered single pinned spiral waves [8] and the pinning mechanism is in literature frequently associated with VF and the difficulty of its termination $[39,10,87,64,23,34]$. In spite of this, little is known about the unpinning of multiple spiral waves in excitable media.

The following pages contain the manuscript

\footnotetext{
${ }^{1} \mathrm{VT}$ has a lower frequency compared to VF, but a higher one than the NSR.
} 
H. tom Wörden, U. Parlitz, and S. Luther. Simultaneous unpinning of multiple vortices in two-dimensional excitable media. Physical Review E, E 99, 042216, 2019.

in which a more detailed introduction into this topic is provided and where my coauthors and I investigate the unpinning of multiple spiral waves in excitable media. The contributions to this manuscript are as follows:

Henrik tom Wörden planned and conducted the research and wrote the manuscript. Ulrich Parlitz and Stefan Luther supervised the research and revised the manuscript.

In this numerical work, I used the simulation framework MediaSim [13] which provides implementations of the Monodomain Model, the Phase Field Method, and the Barkley Model. I wrote software that allows the conduction of the required simulations. E.g. this software creates the initial conditions and boundary conditions for the various configurations that were used (different numbers of spirals, different domains sizes, different pulse sequences, etc.) and manages the appropriate storage of parameters, data and results. Additionally, I wrote the software that was used to analyze the simulation data and results. Here, the implementations to detect phase singularities, detect pinned spirals and estimate the position of a wave front at a heterogeneity use or are based on implementations by Tariq Baig-Meininghaus and Sebastian Berg. 
Place holder for the manuscript

H. tom Wörden, U. Parlitz, and S. Luther. Simultaneous unpinning of multiple vortices in two-dimensional excitable media. Physical Review E, E 99, 042216, 2019. DOI: 10.1103/PhysRevE.99.042216 
Place holder for the manuscript

H. tom Wörden, U. Parlitz, and S. Luther. Simultaneous unpinning of multiple vortices in two-dimensional excitable media. Physical Review E, E 99, 042216, 2019. DOI: 10.1103/PhysRevE.99.042216 


\section{Experiments}

The experimental data on the electric dynamics of the heart was obtained in perfusion experiments with excised, whole pig and rabbit hearts. The main concept of the experiments is to keep an excised heart under physiological conditions, measure its electric dynamics using optical mapping while perturbing and controlling the electric dynamics of the heart using local and far field electric stimulation.

The experimental setup existed already in the work group when I started my work and was described in great detail before [38]. During my studies multiple people used the setup and it was continuously enhanced. I contributed to this process and was additionally contributing to or responsible for the design and conduction of the different experiment series that are subject of this study. Conducting the experiments typically required two persons and sometimes two shifts. I contributed to this effort in a large portion of the experiments that contributed data to this thesis.

In this section, I will give an overview and focus on changes compared to the previous description.

\subsection{Langendorff Perfusion System}

In order to keep an excised heart under physiological conditions the tissue needs to be supplied with oxygen, nutrients and appropriate ion concentrations. In the experiments at hand, this is done using a Langendorff perfusion system which was described e.g. in the review by Bell et al. [9].

In a Langendorff perfusion system a heart is perfused retrogradely by connecting the perfusion circuit at the aorta. "With the perfusion buffer flowing retrogradely down the aorta, opposite to normal physiologic flow, the aortic valve is closed under pressure. With a column of perfusion buffer within the aorta, the coronary arterial vasculature is thus filled [...]" [9] and the whole heart is perfused through the vasculature structure.

The setup is designed such that the perfusion buffer is pumped in a closed cycle. Starting from a reservoir container (see fig. 3.1) the buffer is pumped into a container where it is bubbled with carbogen $\left(95 \% \mathrm{O}_{2}\right.$ and $\left.5 \% \mathrm{CO}_{2}\right)$. The buffer flow continues through a heat exchanger into a bubble trap. Below the bubble trap, the heart is connected to the perfusion system at the aorta and suspended into a bath filled with perfusion buffer. Excess liquid leaves the bath via an overflow and is led back into the reservoir tank. The temperature of the perfusion buffer in the bath is kept at $37^{\circ} \mathrm{C}$. 


\section{Experiments}

In experiments with pig hearts Krebs-Henseleit perfusion buffer ${ }^{1}$ is used (see table A.7 for details on the used composition) and Tyrode solution ${ }^{2}$ is used in experiments with rabbit hearts (see table A.8 for details on the used composition). Part of the experiments were conducted in a constant pressure setting where the pressure was controlled to maintain a constant value. In other experiments, the pressure was adjusted in order to keep a constant flow rate or to allow the induction of arrhythmias.

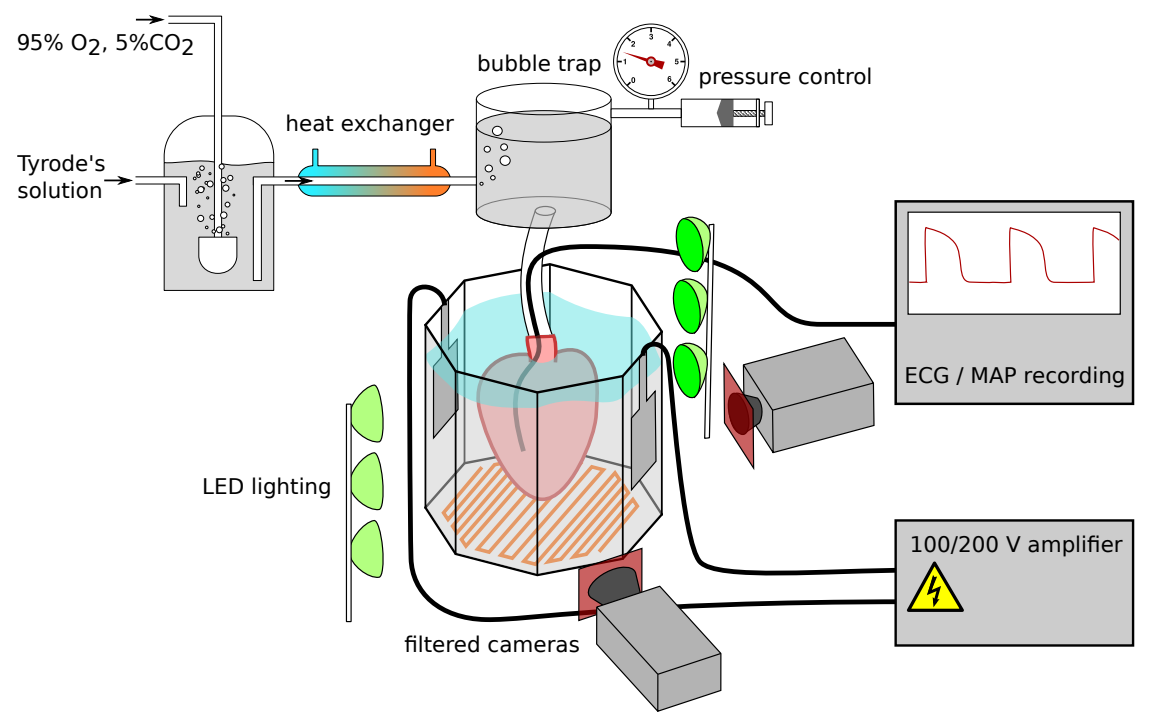

Figure 3.1: Schematic drawing of the experimental setup. Figure from [38] by courtesy of D. Hornung. The figure contains graphics from http: //www.openclipart.org.

\subsection{Optical Mapping}

Voltage sensitive dyes allow to visualize changes of the transmembrane voltage of cardiomyocytes. The key principal is that these dyes can be excited to emit fluorescent light and the emission spectrum of this light changes with the transmembrane voltage of the cells. Two detailed reviews on this subject are [27, 35].

The dye di-4-ANEPPS ${ }^{3}$ is provided to the heart by adding a bolus into the bubble trap. The perfusion buffer distributes the dye in the tissue and the dye binds to the cell membrane of cardiomyocytes. The excitation light is provided through short pass filters (FD1C subtractive Dichroic Color Filter ${ }^{4}$, cutoff at $560 \mathrm{~nm}$ ) from LUXEON Rebel LEDs $^{5}$ (peak wavelength $(530 \pm 10) \mathrm{nm}$ ) which are positioned around the setup.

\footnotetext{
${ }^{1}$ Different compositions of this buffer are reported in literature. See e.g. [4, 9].

${ }^{2}$ Again, different compositions of this buffer are reported in literature. See e.g. [81, 17].

${ }^{3}$ Life Technologies, Darmstadt, Germany

${ }^{4}$ Thorlabs $\mathrm{GmbH}$

${ }^{5}$ LUMILEDS Holding B.V.
} 
The emission light from the dye is recorded with four Evolve® 128 EMCCD cameras $^{6}$ that are positioned around the glass container with a $90^{\circ}$ spacing. Long pass filters (Schott RG-610 ${ }^{7}$ cutoff edge at $610 \mathrm{~nm}$ ) that absorb the excitation light are positioned in front of the lenses of the cameras to prevent a distortion of the fluorescence signal by the excitation light. This is especially important as the change of the emission light intensity due to voltage changes typically amounts to only a few percent of the absolute intensity. The cameras are operated at a speed of $500 \mathrm{~Hz}$ to provide sufficient time resolution. The cameras are controlled and their images are saved using the software MultiRecorder [1] that is developed in the Biomedical Physics Group.

When the heart is moving during imaging, one pixel does not show the same part of the heart tissue on different video frames. Therefore, local time series like an action potential can no longer be obtained. Additionally, due to varying emission light intensities on the heart surface, fractional changes of the signal due to the motion can be much larger than the signal difference due to membrane voltage changes. Even though techniques are being developed to compensate those effects (see e.g. [89, 19]), the only robust and feasable method was to prevent motion of the heart. Thus, the hearts are immobilized by the excitation-contraction decoupler (-)-Blebbistatin ${ }^{8}$ in the here described experiments.

\subsection{Electric Stimulation}

Two different kinds of electric stimulation are used to perturb or control the electric dynamics of the heart: local stimulation and far field stimulation. The local stimulation is done using bipolar electrodes, that are positioned on the heart muscle. When a voltage is applied to those electrodes, a small current flows from one pole to the other through the heart tissue and in doing so it can excite the heart tissue. In all experiments, such a bipolar electrode is used to stimulate the epicardium. When using pig hearts, a catheter (Medtronic Sprint Quattro Secure $\mathrm{S} \AA^{9}$ ) is inserted additionally through the right atrium into the RV. The tip of the catheter is screwed into the tissue and is also used as an electrode for local stimulation.

The far field pulses are applied using panel electrodes, that are positioned at two sides of the bath (see fig. 3.1). The pig bath is larger in size than the rabbit bath, such that the electrodes are separated by $14.3 \mathrm{~cm}$ instead of $9.0 \mathrm{~cm}$. The voltage is applied to the panel electrodes either using a high-power amplifier (BOP $100-10 \mathrm{MG})^{10}$ for voltages up to $100 \mathrm{~V}$ or a custom-built defibrillator (see [38]) that can deliver up to $1000 \mathrm{~V}$.

The electric stimulation devices are controlled using the software Pulsar which is developed in the Biomedical Physics Group. The software allows to define various

\footnotetext{
${ }^{6}$ PHOTOMETRICS, Tucson, USA

${ }^{7}$ SCHOTT AG, Mainz, Germany

${ }^{8}$ BIOZOL Diagnostica Vertrieb GmbH, Germany

${ }^{9}$ Medtronic, Inc., USA

${ }^{10}$ Kepco Inc., USA
} 


\section{Experiments}

shock sequences and even the application of shock sequences that depend on the electric measurements just before the application. This will be described in the following section.

\subsection{Data Acquisition}

The pressure of perfusion at the height of the aorta, the camera trigger, voltage and current of electric stimulation and ECG like signals are recorded using the AcqKnowledge software, BIOPAC acquisition modules ${ }^{11}$ and custom-built BIOPAC compatible amplifier modules ${ }^{12}$. The ECG like signals (for simplicity called ECG signals in the following) show the voltage difference of two electrodes (either $10 \mathrm{~mm}$ EEG Electrode Sintered Silver-Silverchloride $\mathrm{Ag}-\mathrm{AgCl}^{13}$ or electrodes similar to Stimuplex $\mathbb{R}$ Ultra ${ }^{14}$ ) positioned on opposite sides in the bath. The AcqKnowledge software records the above mentioned signals continuously during the whole experiment.

Additionally, one ECG signal is recorded by the custom-built Pulsar software using a DAQ acquisition module ${ }^{15}$. The pulsar software allows to record the ECG for some time, make a frequency analysis and apply a shock sequence depending on this analysis. This is done in an automated fashion where the application of the shock sequence starts in less than $0.1 \mathrm{~s}$ after the end of the ECG recording that is included in the analysis.

The recordings presented in this thesis that employed this on-the-fly frequency analysis, use a $1 \mathrm{~s}$ recording of one ECG to which a sinus wave with constant amplitude and cycle length is fitted. The cycle length of the fit is considered to be a measure for the frequency of the dynamics and will be called dominant electric cycle length denoted by $T_{d}^{E C G}$ in the following. The frequency $1 / T_{d}^{E C G}$ will be denoted by $f_{d}^{E C G}$.

\subsection{Animal Model}

As mentioned before, the experiments were conducted with excised rabbit and pig hearts. Female New Zealand White Rabbits were used. In some experiments, a cryo zone $^{16}$ was created centrally on the LV in order to promote the maintenance of VF.

Female Göttingen Minipig were used with two different heart disease models:

\footnotetext{
${ }^{11}$ BIOPAC Systems Inc., USA

${ }^{12}$ Modules are designed and built by Laura Diaz.

${ }^{13}$ Neurofeedback-Partner GmbH, Germany

${ }^{14}$ B. Braun Melsungen AG, Germany

${ }^{15}$ National Instruments Corporation., USA

${ }^{16}$ The cryo zone was created by two times $10 \mathrm{~s}$ contact to the heart surface with a $6 \mathrm{~mm}$ diameter metal piece cooled to the temperature of liquid nitrogen.
} 
1. Chronic Myocardial Infarction: This model was previously described by Kupatt [49]. "Briefly, a balloon was placed in the left anterior descending artery (LAD) distal to the bifurcation of the first diagonal branch and inflated with 4 atm (0.41 MPa)." [37] This leads to the lack of blood supply in a region of heart tissue that in response degenerates to scar tissue (see also [49]).

2. Chronic Heart Failure: A stent is inserted into the aorta that increases the flow velocity and thereby the pressure in the heart chambers. The heart muscle becomes increasingly fibrotic in response to this and is only able to eject a smaller fraction of the blood in the ventricles after a few month. The detailed animal model will be published by Rabea Hinkel, Claudia Richter and co-workers.

All animal procedures were conducted in accordance with applicable German animal welfare law.

\subsection{Experimental Procedure}

Most ex-vivo experiments with pig hearts were preceded by in-vivo experiments in which defibrillation techniques were studied. At the end of the in-vivo experiments the pigs were sacrificed and the hearts were excised. All data that is presented and analyzed in this thesis stems solely from the ex-vivo parts of the experiments. I will therefore mostly restrict the following description to the ex-vivo part of the experiments.

Before excision, heparin and cardioplegia was given to both pigs and rabbits in order to prevent blood coagulation and deterioration of the heart tissue during transport to the lab and preparation of the heart for the perfusion setup. The heart is connected to the perfusion setup, flushed with perfusion solution in order to remove remaining blood and is then suspended in the bath. Afterwards, the voltage sensitive dye (pig hearts: $1 \mathrm{ml}$; rabbit hearts: $0.6 \mathrm{ml}$ ) is given as a bolus into the bubble trap and the blebbistatin (pig hearts: $10 \mathrm{ml}$; rabbit hearts $6 \mathrm{ml}$ ) is added to the perfusion buffer reservoir tank. Additional blebbistatin may be added to the perfusion buffer when residual motion is too strong. After the motion comes to a halt the electrode for local stimulation is placed on the heart surface and measurements can start.

The measurements typically consist of electricly stimulating the heart, possibly after induction of an arrhythmia. After a consecutive sequence of measurements during VF or $\mathrm{VT}$, the arrhythmia is terminated and a resting time of 5 min allows the heart to recover before the next arrhythmia. If multiple defibrillation methods are tested, the methods are selected in a randomized manner. Typically the energy of defibrillation techniques is adjusted to an step-up-down protocol (see section 4.2.1).

In the course of an experiment the optical signal quality often decreases. Sometimes this can be compensated by adding additional dye. However, the tissue overall deteriorates during the experiment. For example, the amplitude of the ECG signal decays. Therefore, experiments are typically ended when the signal quality becomes 


\section{Experiments}

too bad or the activity of the heart does not allow the planned measurements (e.g. no arrhythmia induction possible or no arrhythmia termination possible).

\subsection{Data Management}

While the data acquired in the here described experiments can still be stored on standard hard drives ( $\approx 500 \mathrm{~GB}$ per experiment), we face typical challenges in our group that are related to data management. On the one hand e.g. video recordings with a size of $20 \mathrm{~GB}$ or more need special consideration when processing them as they cannot simply be loaded into memory and the network transfer of this data takes considerable time such that it cannot be read arbitrarily often. On the other hand the interconnectivity of the data often makes the analysis complex and challenging.

Due to these challenges, it is crucial to keep track of the processing state: It is immensely power and time consuming to redo the preprocessing of e.g. video data always from scratch, thus intermediate results need to be stored and it must be possible to reconstruct how those were created. This implies the storage of software and source code versions as well as used parameters. These steps are crucial to allow reproducibility and they are therefore required by good scientific practice.

The open source software CaosDB [30] has been developed in the Biomedical Physics Group (BMPG) over the last years and addresses these challenges. I contributed to the software development and especially to the creation of tools for the insertion of experimental data. Nowadays large parts of the experimental data of the BMPG are stored in CaosDB. CaosDB is a Research Data Management System that was designed for scientific environments. In particular, it is designed around the concept of semantic data modeling. This means that the connections that the data naturally has can be represented and an inheritance capability, similar to the one of object oriented programming, allows to give a meaning to stored records. For example, Experiment records exist in our dataset but also LEAP Experiment records. The latter are Children, i.e. a special subtype, of the Experiment record. This enables very flexible and powerful searches on the stored data. Further details on CaosDB are provided in [31].

The analysis presented in this thesis made heavily use of CaosDB. Especially, the access to specific datasets via a Python client proved to be more efficient than previous workflows and supported the automated analysis of almost a thousand defibrillation attempts. Apart from experimental data, data from numerical simulations, analysis results and publications can be stored and inter linked in CaosDB. For example, the whole numerical workflow from chapter 2 is archived in this way. Figure 3.2 shows an exemplary query in the CaosDB web interface that presents an analysis result of the aforementioned numerical workflow. 


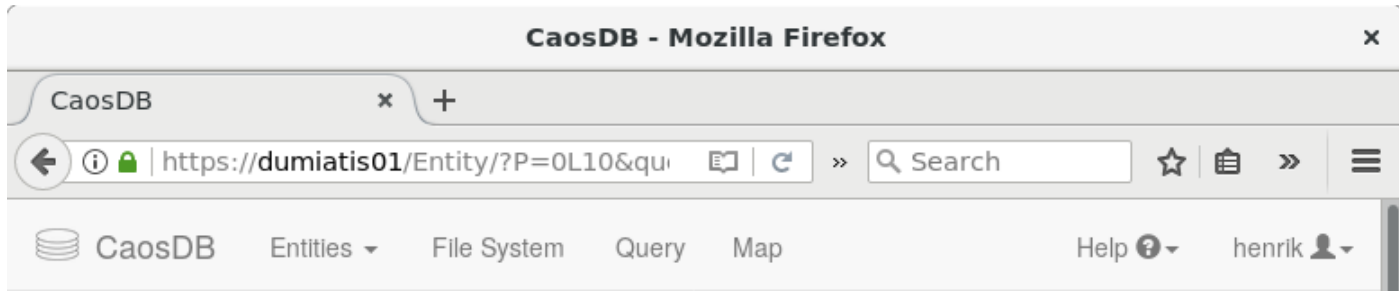

Query:FIND Record AnalysisResult WHICH HAS BEEN CREATED BY ME

Results:10

ParseTree:(cq FIND (role Record) (entity AnalysisResult) (entity_filter (which_exp WHICH HAS BEEN) (filter_expression (transaction CREATED (transactor BY ME)))) 〈EOF $\rangle$ )

description: These are the results of several simulations with a single pinned spiral where the period of such spirals is investigated. Both the dependency of the period on the radius and the transients until a stable period is reached when starting from a constructed initial condition are analysed

update

\begin{tabular}{|c|c|c|c|c|c|c|}
\hline Parents & & & & & & \\
\hline \multicolumn{7}{|l|}{ SimulationAnalysisResult } \\
\hline \multicolumn{7}{|l|}{ Properties } \\
\hline ( ResultPlot & () & 279532 & 279533 & 279534 & 279535 & \\
\hline ( AggregatedData & () & 279529 & 279530 & 279531 & & \\
\hline @ Simulation & () & 279527 & & & & \\
\hline Comments & & & & & & add new comment \\
\hline
\end{tabular}

Figure 3.2: The result of an exemplary query in the web interface of CaosDB is shown. Below the query (marked with green) some debug information (ParseTree) is shown which can be ignored here. Thereafter follows the first result: a record (identified by $\mathrm{R}$ in a red box) with a description. The number on the right is its ID. Below this the Parent type is shown followed by properties of the record. Here, the properties refer to other records stored in CaosDB (identified by their IDs), e.g. to plots that are part of the analysis, to the data that was used and to the simulation that created the data. Note that the query specified the type AnalysisResult but the result record has the type SimulationAnalysisResult, a Child of the latter. 


\section{Experiments}

\subsection{Preprocessing of Optical Mapping Video Data}

The optical mapping video data is preprocessed in a series of steps in order to make the electric activity visible and to reduce noise. The various preprocessing steps described in the following are used commonly. For example, Laughner et al. [51] describe those steps in a review on processing of optical mapping data. However, every processing step comes with advantages and disadvantages such that their use has to be carefully considered for each analysis. The particular sequence of preprocessing thus differs among researchers, among analyses and possibly among datasets. In order to clarify how the data that is present in this thesis was processed, I will describe the preprocessing in the following. Figure 3.3 supports the following description by presenting the different stages of filtering for both a frame of the video data and two exemplary time series. The shown data is an excerpt from a longer recording of a fibrillating rabbit heart.

The quantity of interest is the transmembrane voltage. However, the voltage sensitive dyes do not allow a measurement of absolute transmembrane voltages as described in section 3.2. Nevertheless, if the fluorescent light is filtered correctly, relative changes in the transmembrane voltage are reflected proportionally in relative changes of the light intensity ( $10 \%$ change in fluorescence per $100 \mathrm{mV}$ change in transmembrane voltage [35]). Still, I will not assume such a linear dependency in the following analysis but only a negative correlation ${ }^{17}$.

Consequently, in the unfiltered video data (see fig. 3.3 (a)) it is not possible to see the electric activity on the heart surface. However, when for each pixel the mean of the corresponding time series is calculated and subtracted from that pixel's value in each frame, then the electric activity on the heart surface becomes visible (see right image in fig. 3.3 (b)). For convenience the signal is inverted such that an excitation increases signal values which is the typical way to depict an action potential.

In the right image of fig. 3.3 (b) large pixel wise fluctuations can be seen. These fluctuations are due to noise. ${ }^{18}$ Thus, a spatial smoothing with a gaussian kernel is applied to each image of the video data and does reduce this noise efficiently. However this naturally also blurs the image (see fig. 3.3 (c)). The standard deviation of the gaussian kernel is set to 1 pixel. The reduction of noise is also clearly visible when the corresponding time series are compared to those without smoothing.

In order to further reduce the high frequency noise in the time series in fig. 3.3 (c) and to remove drifts in the signal a band pass filter is applied to the time series of each pixel. Figure 3.3 (d) shows that this removes the high frequency noise efficiently. A first order Butterworth band pass filter with the characteristic frequencies at $0.2 \mathrm{~Hz}$ and $30 \mathrm{~Hz}$ is used because the power spectrum during VF typically has the most power within the range from 4 to $15 \mathrm{~Hz}$.

\footnotetext{
${ }^{17}$ The emission spectrum shift of the used dye (di-4-ANEPPS) during depolarization is such that the intensity of the fluorescent light is decreased.

${ }^{18}$ The expected scale of patterns in the electric activity is much larger. See also fig. 3.3 (b).
} 

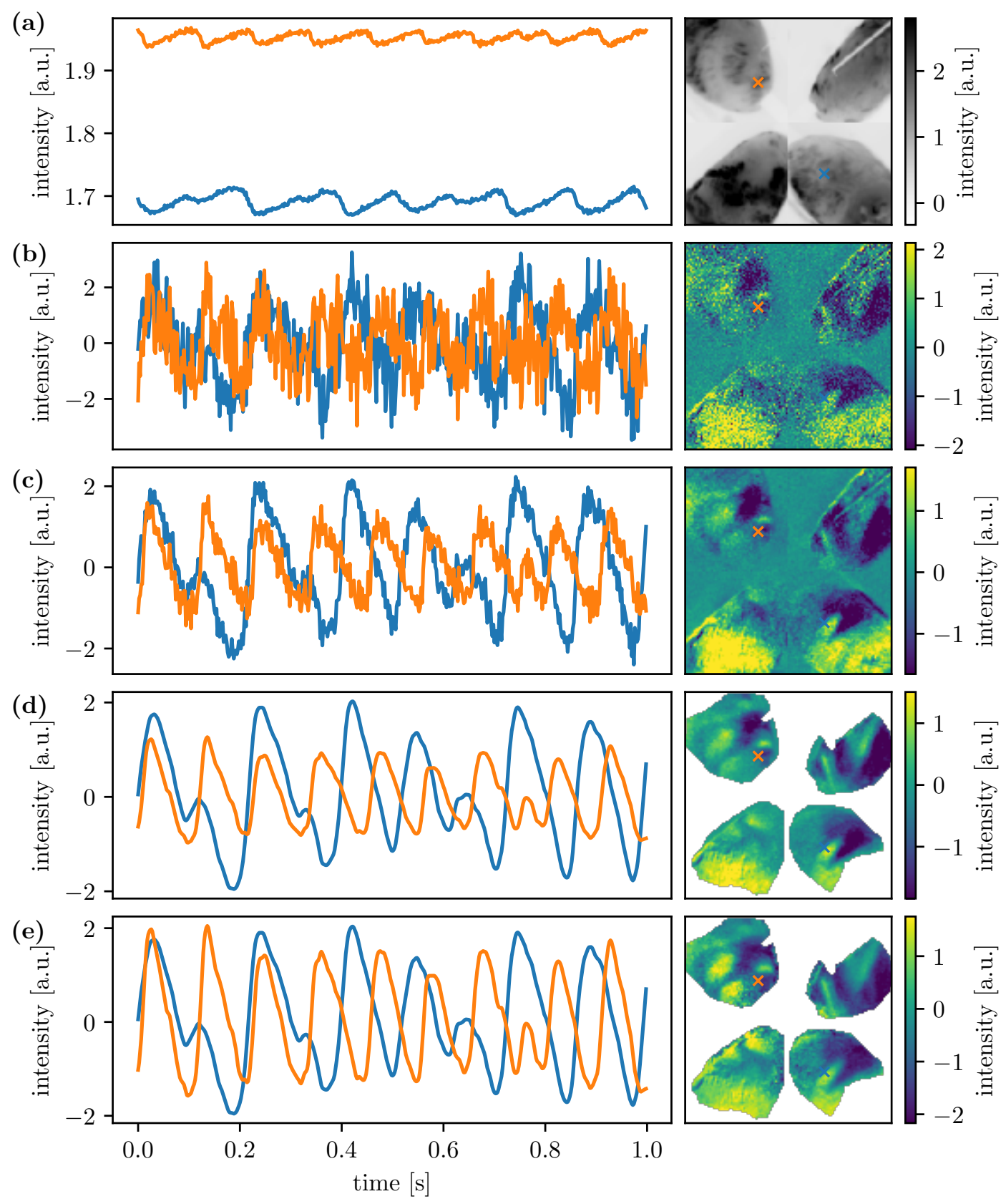

Figure 3.3: The different stages of the preprocessing of optical mapping video data is presented. The top row shows the raw data and each following row shows the data after another preprocessing step. Exemplary time series are shown on the left with the corresponding pixels marked on the right. The processing steps are: (b) inverted and with point wise subtracted mean value (c) gaussian spatial smoothing (d) band pass filter (e) point wise normalization. The value range in (b) to (e) was rescaled for uniformity. Please refer to the main text for details. 


\section{Experiments}

The two curves in the left diagram of fig. 3.3 have different amplitudes. In the analyses of this thesis, I am more interested in state changes, e.g. from excitable to excited, and not so much in the amplitude of the transmembrane voltage change. In order to prevent a varying weighting of local signals, a normalization of each time series is performed. In Figure 3.3 (e), the curves have therefore comparable amplitudes. This additionally enhances the visibility of activation patterns which can be seen at the two activated spots in the upper left which are more clearly visible in image (e).

This whole procedure is applied to every dataset. However, the data stems from experiments within a time span of almost two years. The setup evolved within this time. For example, a power supply instead of a battery is nowadays used to operate the LEDs. Sometimes the batteries did not provide a constant voltage which lead to a drift in the optical signal. The band pass filter that is applied in the preprocessing removes this drift, but it can create artifacts in other signals, e.g. when VF changes to NSR. With this example, I would like to illustrate that the preprocessing performs well in most cases but not in all. The automated analysis that will be described in the next chapter does not allow to check every single dataset because thousands of videos are analyzed. Thus the data may contain recordings that have poor signal quality or where preprocessing caused artefacts.

The preprocessing was mostly done employing PythonAnalyser, a software developed in the group that implements various filters and allows to conveniently load the video files. The appropriate use of this generic tool and the implementation of software that allows the application to the huge amount of data was part of the work at hand. 


\section{Results}

In the thesis at hand, I investigate the control of excitable dynamics during VF in rabbit and pig hearts. In the first part of this chapter, I will present my results on the control of these dynamics using periodic sequences of electric far field pulses. Periodic far field pulses have been proposed as means to terminate VF (see section 1.5). However, I will not only consider whether those periodic sequences can terminate VF but more importantly, how the dynamics of VF are changed and how the effects of the control depend on parameters of the pulse sequences and on the state of the heart.

In the second part, I will investigate a novel defibrillation technique. The design of this technique is inspired by the results on simultaneous unpinning of multiple spirals presented in Chapter 2. However, the technique can also be seen as an attempt to exploit the control by periodic pulses, described in the first part of this chapter, in order to enhance defibrillation.

In the third part, I will describe the recordings of a two defibrillation attempts in detail and illustrate what can be learned from the observed dynamics together with the knowledge, that is gained in the first two parts of this chapter.

The results that will be discussed throughout this chapter were created with analysis software that I wrote.

\subsection{Control by Periodic Pulse Sequences}

Different kinds of periodic sequences of electric far field pulses have been proposed as means to terminate VF (see section 1.5). However, the mechanism that leads to the termination of VF when applying such pulses is unclear and the literature is lacking a quantitative analysis on the effect of such pulses on the dynamics of VF. I will therefore investigate in this section when periodic pulses lead to the termination of $\mathrm{VF}$, how the effect of such pulses on the dynamics of VF can be characterized and quantified and eventually how this effect depends on parameters of the pulse sequences and on the electric dynamics in the heart.

While some proposed techniques for terminating fibrillation apply multiple pulses within a cycle of the cardiac activity [53], others employ pulse sequences with a period close to this cycle length [55]. The pulse sequences that will be investigated in this section are similar to the latter and therefore analogous to the control that was used in chapter 2 . Their energy is about $1 / 100$ to $3 / 4$ of the energy of conventional single 


\section{Results}

shock defibrillation in experiments with rabbit hearts and about 1/3600 to $1 / 36$ of the single shock energy in experiments with pig hearts.

In order to investigate the effect of periodic far field pulse sequences a special kind of long pulse sequence was applied in experiments during VF that will be called Synchronization Map in the following. The analysis in this section will widely make use of the corresponding optical mapping data. In the following, the influence of two important parameters of periodic pulses sequences on the control is studied: the influence of the frequency of the pulses and the influence of their voltage.

Therefore, a Synchronization Map was designed to consist of several blocks of periodic pulses, each having a higher voltage $U$ than the previous one (see fig. 4.1). However, due to technical limitations the pacing period $T_{p}$ is the same in all blocks and thus multiple Synchronization Maps with different pacing periods are recorded in a single experiment in order to allow conclusions on the influence of the frequency.

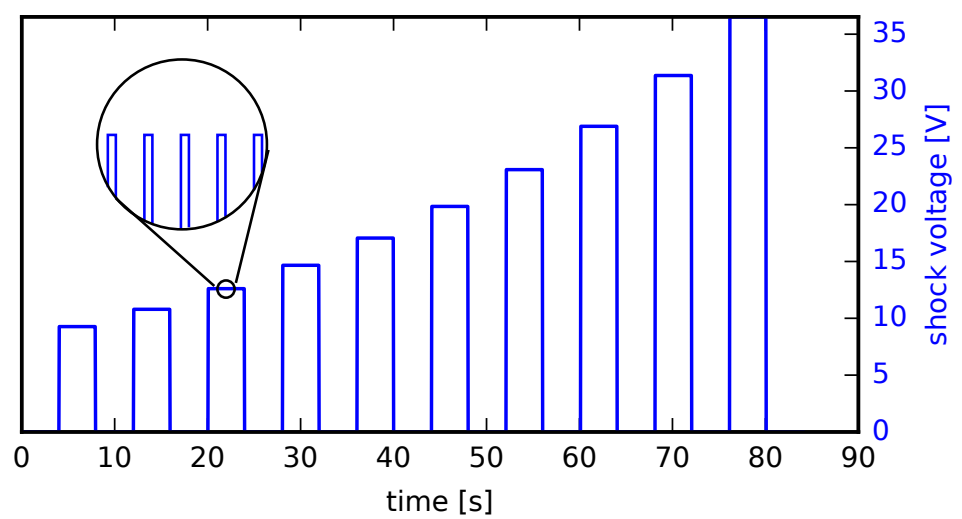

Figure 4.1: An illustration of the Synchronization Map pulse sequence is shown. A Synchronization consists of multiple blocks separated by a gap of a few seconds and each block is a periodic sequence of around 40 pulses. All blocks have the same pacing frequency while the voltage of the pulse increases with each block.

The voltage is applied between the two plate electrodes in the bath (see section 3.3). The Synchronization Maps that will be considered in the following have around $N_{B}=10$ blocks with voltages spaced logarithmically from 5 to $40 \mathrm{~V}$ (20 to $100 \mathrm{~V}$ ) in experiments with rabbit (pig) hearts. This amplitude range is chosen because pulses with a voltage of the lower end cause no visible change in the dynamics, while the upper end corresponds to activations of a large portion of the tissue. The blocks typically consists of $N_{p}=40$ pulses and are usually separated by $T_{g a p}=4 \mathrm{~s}$. The pacing period $T_{p}$ was set to roughly 0.8 to 1.2 times the dominant frequency of the VF (see fig. 4.6), which was estimated from a visual inspection of the frequency spectrum during the experiment. For details on the choice of parameters please refer to tables A.1 and A.2. The datasets consisting of those Synchronization Maps recorded in experiments with rabbit and pig hearts will be referred to as $S y n c R$ and $S y n c P$, respectively. 
Please note that in the following analysis often single blocks within an Synchronization Map are considered. This also implies that when a single pulse sequence is related to the dynamics of the VF, the dynamics just before the onset of that sequence are used for calculations and not the dynamics before the whole Synchronization Map. Because a termination of the VF during the application of a Synchronization Map will change the dynamics drastically. Those blocks that are applied after the termination are therefore excluded from the analysis. Termination of VF within Synchronization Maps are detected automatically using an algorithm that checks whether the application of a pulse sequence is followed by a period with no activity on the heart surface and a sufficient duration or whether the subsequent activity resembles one caused by NSR $\mathrm{VF}$ is considered to be terminated when for a duration of $1 \mathrm{~s}$ or longer no activity or NSR is present. While manual inspection showed that most recordings are classified correctly, misclassifications cannot be excluded.

\subsubsection{Termination of Ventricular Fibrillation with Periodic Pulses}

While the standard technique for defibrillation is still the application of a single, high-energy, electric far field shock (see section 1.3), periodic stimulation with electric far field pulses have been discussed as means for control of arrhythmias of the heart and especially as means to terminate $\operatorname{VF}[55,52,53]$. The mechanism that leads to termination of $\mathrm{VF}$ when applying periodic far field pulses is however still being disputed (see section 1.5). In this section, I will therefore shed some light on the questions when and how periodic far field pulses terminate VF.

Luther et al. [55] pointed out, that the maximum of the activated area, i.e. the area of the heart surface where the optical signal is above a certain threshold, is increased during the pulses of LEAP demonstrating an increased synchronization of the tissue ${ }^{1}$. Additionally, Ji et al. [44] postulated that "When LEAP failed, it was owing to a lack of full synchronization [...]".

Figure 4.2 shows the activated area during a sequence of periodic pulses in a Synchronization Map recording of a fibrillating rabbit heart. Similar to the findings that Luther et al. [55] describe ${ }^{2}$, the pulses increase the activated area. However, here a larger number of pulses is applied compared to the example in [55] and the maxima of the activated area that are caused by the pulses do not increase monotonically. However, once the activation reaches $100 \%$ each following pulse causes again full activation. This particular sequence of pulses leads to the termination of the VF as can be seen by the last two peaks in the activated area. The absence of high frequency fluctuations shows that VF was terminated and the two peaks correspond to activations from NSR. The here depicted sequence consists of 40 monophasic, rectangular pulses with $5 \mathrm{~ms}$ pulse width, a cycle length of $0.1 \mathrm{~s}$ and a voltage of $36 \mathrm{~V}$.

\footnotetext{
${ }^{1}$ Please note, the optical signal reflects changes of the transmembrane voltage. A high value in the processed signal is assumed to correspond to depolarized tissue. Thus if the optical signal is above some threshold, the tissue is then said to be excited or activated.

${ }^{2}$ Please note, that the example in [55] is for the termination of AF and there are many differences in experimental preparation and settings, e.g. the voltage of pulse.
} 


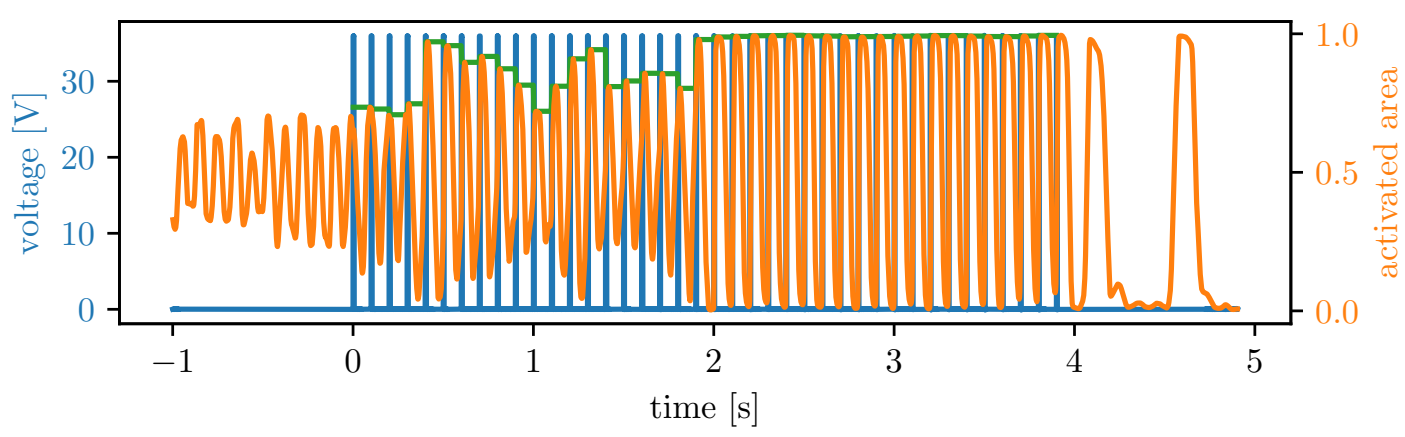

Figure 4.2: The activated area is depicted during a sequence of periodic, electric far field pulses. The data was recorded from a rabbit heart in VF. The activated area is increased by the shocks and reaches $100 \%$ after approximately $2 \mathrm{~s}$. This means that the whole heart is excited after each shock for the remaining $2 \mathrm{~s}$. The two peaks after the shock sequence indicate that VF is terminated and NSR started.

As described in section 1.4, a high-energy single shock basically activates the whole tissue and thereby terminates VF. The above described recording is an example of how periodic pulses can similarly reach full activation iteratively and terminate VF (see also [33]).

Let us consider the maximum activation that is caused by a pulse: In fig. 4.2 the maximum activation in the interval from the onset of one pulse until the next pulse is depicted in green. While this measure will reflect a large activation caused by an electric far field pulse, a causality is not assured: In some cases the maximum activation might not be caused by the previous pulse. For example, in fig. 4.2 the activated area decreases during the first pulse and the maximum is only reached just before the next pulse. Thus this detected maximum is not directly caused by the first pulse. However, this will only occur in the beginning of a pulse sequence or if pulses only have a small effect and large activated area values are caused by the basically unperturbed dynamics. I therefore consider this quantity useful for measuring the effect of periodic pulses. In the following, the maximum fraction of the activated area within one cycle length of the pacing will be called maximum activated area. For the sake of simplicity in the analysis, the beginning of the intervals within which the maximum is considered do not have to coincide with the onset of pulses. This will however only slightly influence the analysis since long pulse sequences will be considered in the following.

We saw in the above example how a simultaneous activation of the whole heart tissue was followed by the termination of VF. Does a periodic pulse sequence only terminate VF when a high maximum activated area is reached within the sequence? Figure 4.3 shows a stacked histogram where the number of blocks of Synchronization Maps, i.e. single periodic pulse sequences, is depicted according to their respective mean maximum activated area in the last eighth of the pulse sequence. The part of pulse sequences that lead to the termination of VF is colored in blue, while the 


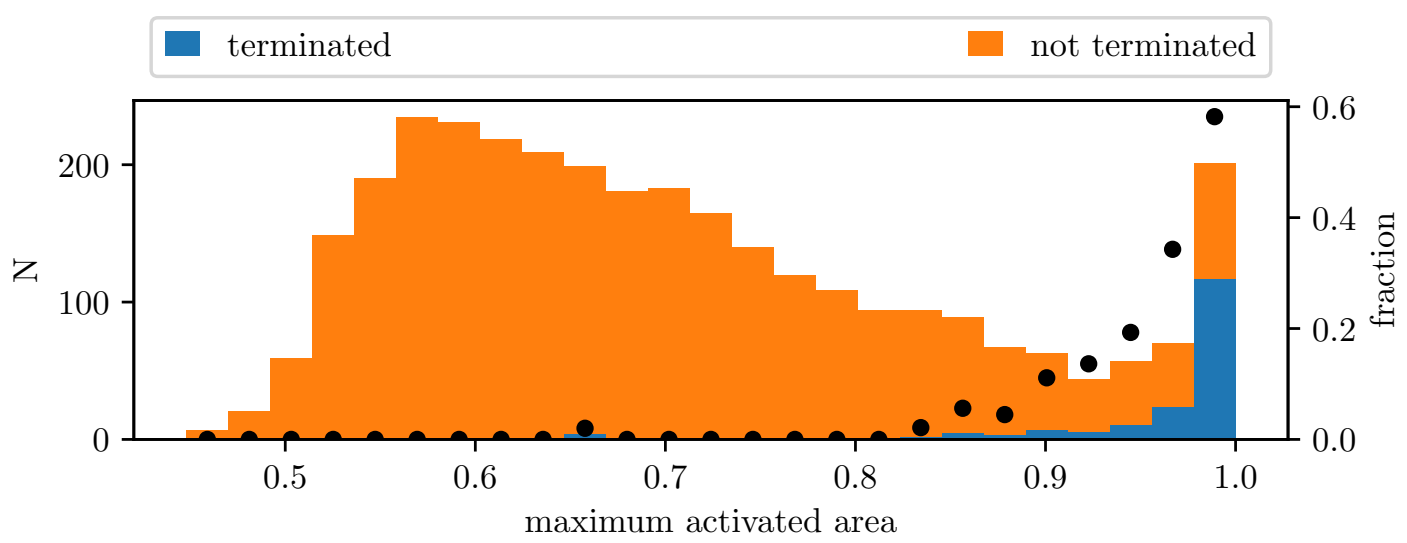

Figure 4.3: A stacked histogram depicts the number of blocks of Synchronization Maps with a certain maximum activated area. The part of pulse sequences that lead to the termination of VF is depicted in blue; the complement in orange. The fraction of terminations is indicated by black dots. Notably, large fractions of termination only occur for large values of the maximum activated area.

complement is colored in orange. Overall, only a small number of pulse sequences lead to the termination of VF because the voltage of many blocks is comparably small $^{3}$. The fraction of sequences which lead to termination (depicted by black dots) increases towards a maximum activated area of 1 . Please note, that this dataset is very heterogeneous: The pulse sequences have different voltages, cycle lengths and pulse lengths and the data is from experiments with rabbit and pig hearts. Still, large fractions of termination are only found for very high values of the maximum activated area.

Thus fig. 4.3 indicates that a large maximum activated area might be necessary for the termination of VF. However, it is also possible that shorter pulse sequences would also have terminated the VF and the superfluous pulses cause the high values of maximum activated area while not counteracting the termination. In this case, the maximum activated area would not be a condition for termination but an indication thereof.

If a large maximum activated area is necessary for the termination of VF with the here considered periodic pulses as it was originally postulated in the critical mass hypothesis for single shocks [91], then this would suggest that also the termination mechanism is similar. The same arguments that go with the critical mass hypothesis can be applied in this situation: When sufficient cardiac tissue is activated such that fibrillatory waves can no longer propagate, then VF is terminated.

\footnotetext{
${ }^{3}$ As a Synchronization Map is stopped after the termination of VF and only blocks before the termination are used in the analysis, the range of voltages is chosen such that typically only the highest voltages sometimes lead to the termination of VF.
} 


\subsubsection{Area Activated by Different Pulsing Frequencies and Pulse Strength}

Of course, the effect of periodic pulse sequences depends on many factors such as voltage, pulse shape, cycle length and so forth. It is known that a sufficient high voltage can excite the whole tissue, thus a sequence with such a voltage will have a maximum activated area of 1 after each pulse. On the other hand, pulses with very small voltages will not have any effect on the dynamics of VF. Also, as mentioned in the introduction (section 1.4), the number of recruited virtual electrodes increases with electric field strength and the control is exercised at additional locations in the heart. Thus, an intuitive guess is that the effect of the pulses and the maximum activated area that is reached increases with the voltage of the pulse sequence.

As every excitable medium, heart tissue cannot be excited at an arbitrary rate. Thus pacing sequences with too high frequencies will not be able to cause large activations ${ }^{4}$. However, apart from this, it is unclear whether the effect of the pulse sequences depends on the pacing frequency.

Let us start to investigate the influence of these two parameters by comparing the maximum activated area caused by different pulse sequences, a measure that was above shown to be an important indicator for whether a periodic pulse sequence leads to the termination of VF or not. Figure 4.4 shows how the maximum activated area averaged over the last eighth of blocks of Synchronization Maps depends on pacing frequency and voltage. The figures (a) and (b) depict data recorded from rabbit hearts while figures (c) and (d) correspond to data from experiments with pig hearts. The data points do not lie on a regular grid because frequencies where chosen depending on the dynamics of the VF and different ranges of voltages were used. Additionally, some Synchronization Maps needed to be aborted before completion when a block led to the termination of the VF. The distribution of data points is depicted on the right in 2D-histograms with a logarithmic color map. Due to large variations of the maximum activated area for similar parameter values, the values in the diagrams on the left are mean values of the data points residing in a disc centered at the respective location. An exemplary disc is depicted in the lower left corner to illustrate size and shape. If less than 25 data points contribute to a mean value, no value is displayed.

For both rabbits and pigs, the expected increase of the maximum activated area with voltage is visible. Additionally, there seems to be a certain frequency (around $9 \mathrm{~Hz}$ ) above which the maximum activated area drops in the data recorded from pig hearts. As mentioned above, this upper limit probably exists because it was not possible to excite the heart tissue at a larger frequency. In the data corresponding to rabbit hearts, there is no such upper limit visible. Notably, except for the upper limit in the data from pig hearts, there is no strong dependency on the pacing frequency visible ${ }^{5}$,

\footnotetext{
${ }^{4}$ Unless e.g. every second pulse activates the tissue.

${ }^{5}$ In the diagram corresponding to data from rabbit hearts, there is an increased maximum activated area at $f_{p} \approx 11 \mathrm{~Hz}$ and $30 \mathrm{~V}$. However, quite few recordings contribute to these values (compare the histogram on the right).
} 
(a)

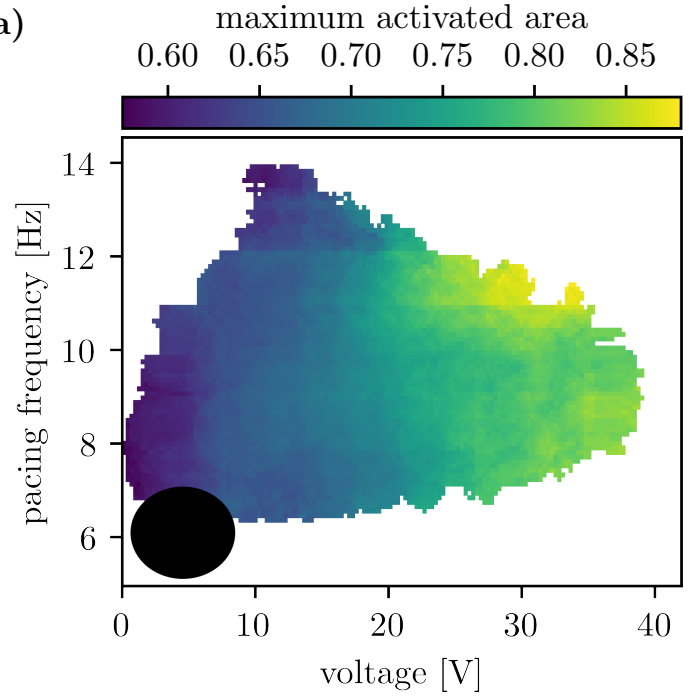

(c)

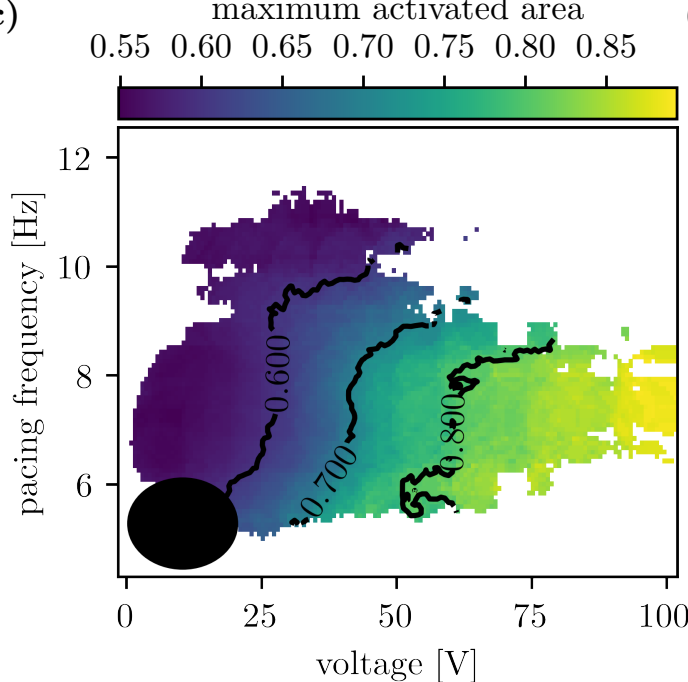

(b)
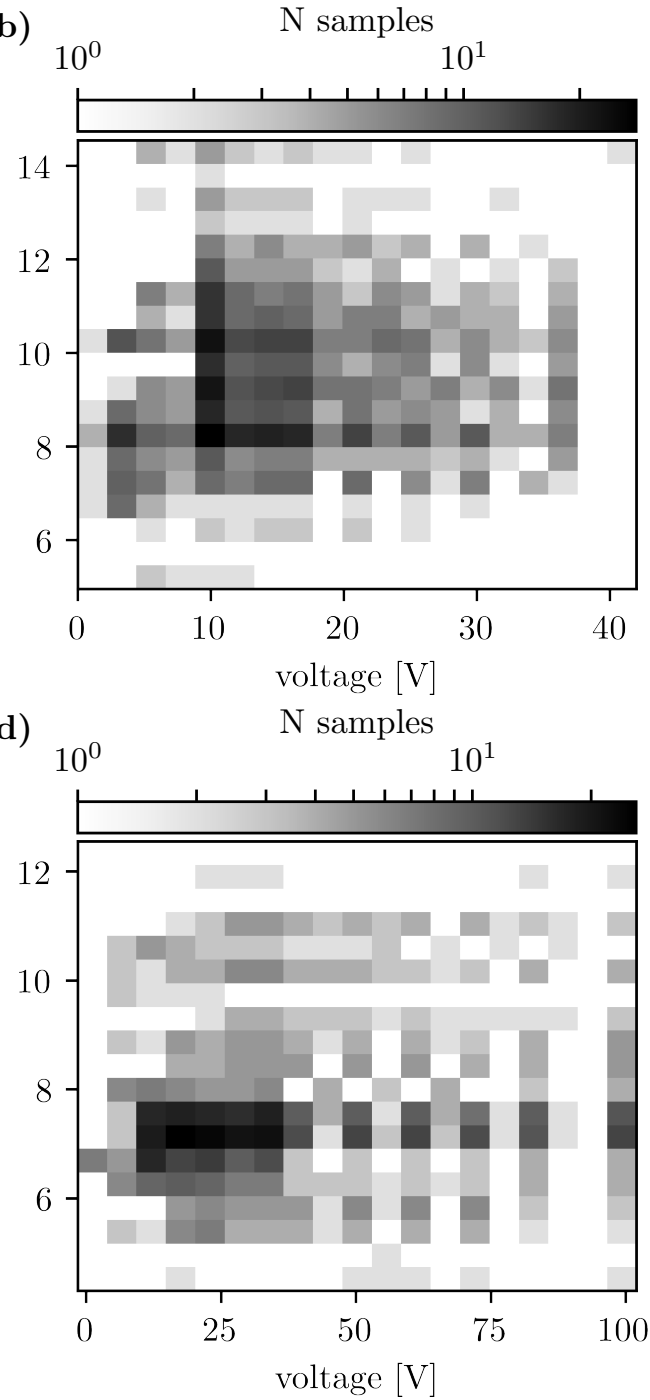

Figure 4.4: These diagrams present the dependence of the maximum activated area on voltage and pacing frequency of periodic pulse sequences applied during VF. The unstructured distribution of data points is depicted on the right in 2D-histograms with a logarithmic color map. On the left, the maximum activated area averaged over the last eighth of the pulse sequences is depicted color coded. At each point in the displayed parameter space the average of all data points that lie in a surrounding disc is depicted. The diagrams (a) and (b) display the rabbit, diagrams (c) and (d) the pig dataset. In the left diagrams, the depicted value is the average of data points in a surrounding disc with a size that is displayed in the lower left in black. If less than 25 data points contribute to a mean value, no value is displayed. Generally, the maximum activated area increases with voltage. And no strong dependency on the pacing frequency is visible except for an upper limit in the data from experiments with pig hearts. 


\section{Results}

i.e. all pacing frequencies (below some value) lead to similar maximum activated area values.

However, it would be surprising, if the maximum activated area does not depend on the pacing frequency in some way: In an excitable medium activation waves of higher frequency can push away slower activations (see e.g. [48]). VF is a dynamic state and for example the frequency of activation changes over time and from experiment to experiment. Thus, instead of depending on the absolute pacing frequency, the effect of a pulse sequence might depend on the particular VF, e.g. its frequency. However, this raises a new question: What is the frequency of a VF? I will shed some light on this difficult question in the next section where I show how the dynamics of VF can be characterized. This will allow to relate the effect of periodic stimulation to the properties of the corresponding VF.

\subsubsection{Characterization of Ventricular Fibrillation}

The complex dynamics of VF (see for example the ECG in fig. 4.5) typically comprise multiple frequencies as the power spectrum in fig. 4.5 indicates (shown below the ECG). Still, the signal has some periodicity and often one would like to express how "fast" the activity is or that it has a higher frequency than something else. The part of the power spectrum, that is shown in fig. 4.5 contains more than $98 \%$ of the total power. Thus, the power spectrum is overall quite narrow and one could argue that the activity shown in the ECG is faster than for example $6 \mathrm{~Hz}$.

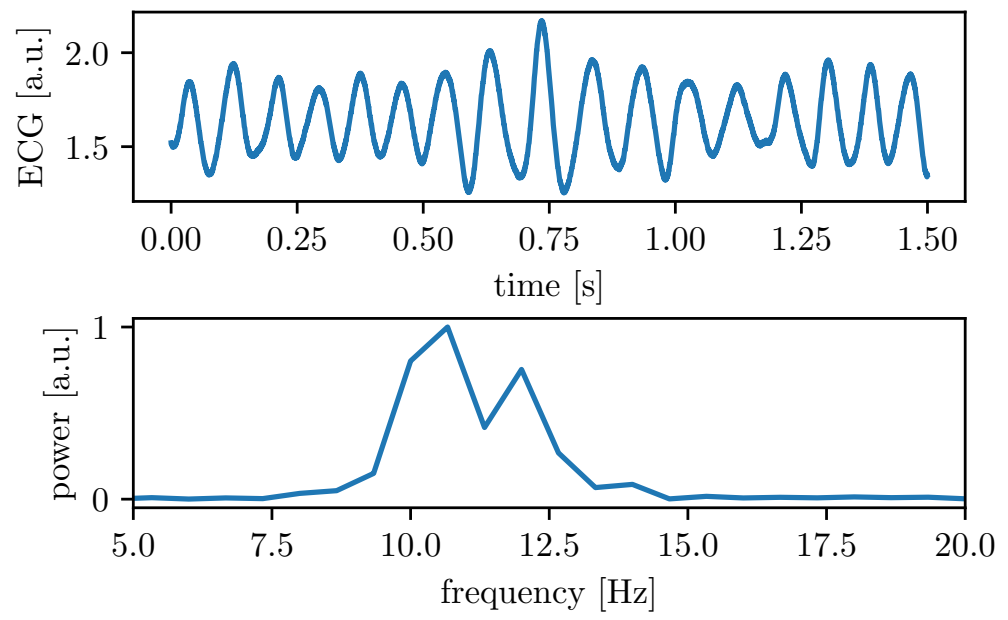

Figure 4.5: The upper diagram shows an exemplary 1.5 s snippet of an ECG recorded from a fibrillating rabbit heart. The relevant part of the corresponding power spectrum is shown in the lower diagram. More than $98 \%$ of the power is contained in the depicted part of the power spectrum.

The term dominant frequency is often used for the frequency of the highest peak in the power spectrum and it can be seen as a characteristic frequency of the VF. 
The dominant frequency allows to make comparisons as the one above and thus allows for example to compare the frequency of the VF to the pacing frequency. There are however several difficulties when using the dominant frequency. First, the dominant frequency might not be well defined, if there are two or more peaks of similar magnitude or if the spectrum is very broad. Second, the dominant frequency can change over time (see e.g. [18]).

This naturally raises the question for the appropriate window length of the Fourier transform to calculate the dominant frequency. While the use of longer time series increases the frequency resolution, windows that are larger than the time scale on which the dominant frequency changes will lead to the loss of this temporal information. In order to resolve the change of the frequency during pacing and still have an acceptable frequency resolution, a comparably small window length of $1 \mathrm{~s}$ is used in for the fourier transform when determining the dominant frequency. This results in a frequency resolution of $1 \mathrm{~Hz}$.
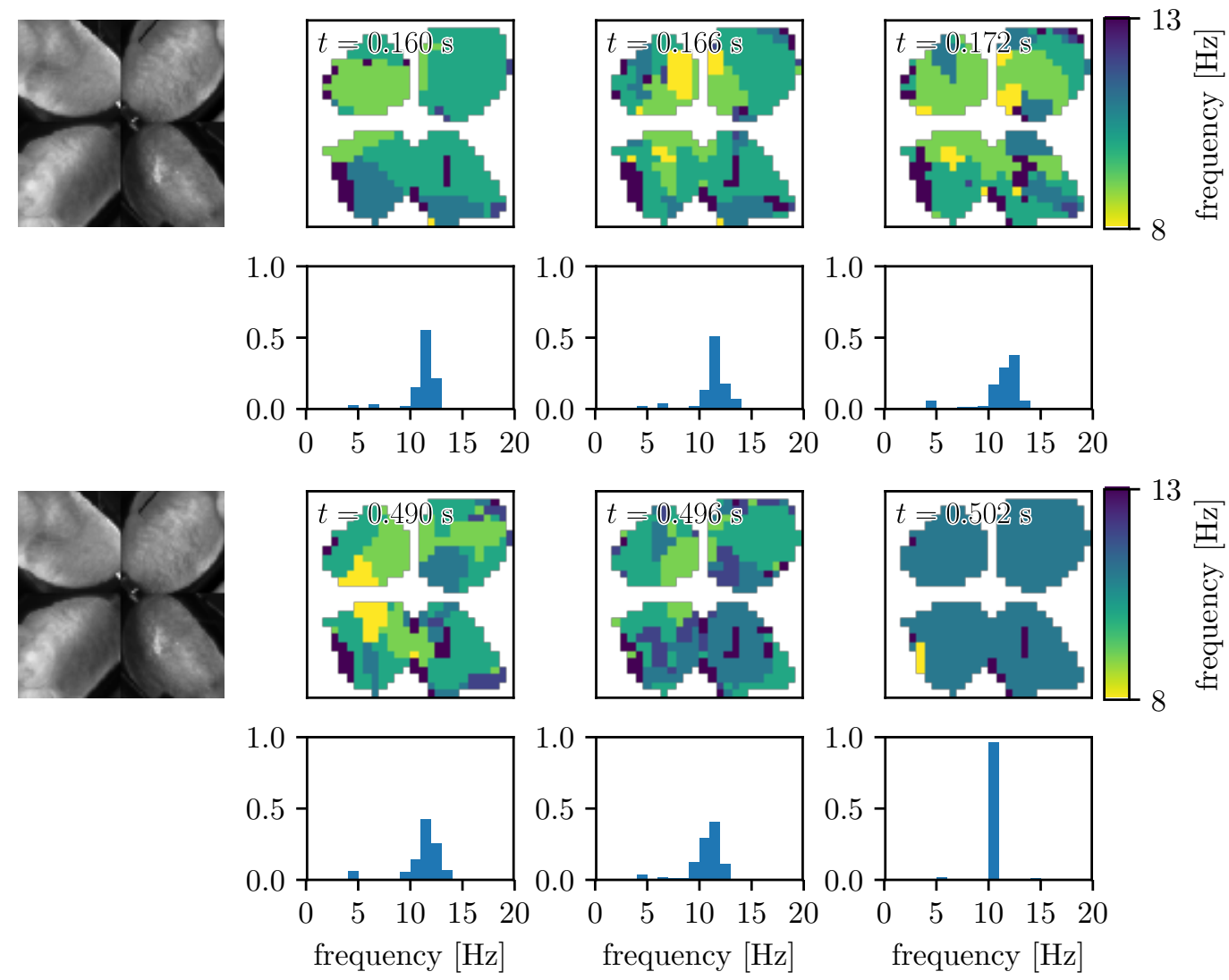

Figure 4.6: Exemplary dominant frequency maps are shown during unperturbed VF (upper row) and during a block of a Synchronization Map (lower row). On the left, an unfiltered frame of the optical signal is shown to illustrate how the data from different views of four cameras are combined and how the heart is oriented in those views. Below the dominant frequency maps, histograms are shown that depict the number of pixels for each frequency. 


\section{Results}

Using the local time series of the optical signal, a dominant frequency can be calculated for specific locations on the heart surface. Figure 4.6 depicts multiple dominant frequency maps (DFMs) that are created by calculating the dominant frequency for a subset of pixels of the heart surface. In the analysis of this thesis only every tenth pixel in every direction, i.e. a hundredth of all pixels, is used in order to reduce computational costs. DFMs are nowadays a standard tool for the analysis of optical mapping data. For example in [80], they were used to investigate the temporal changes and regional differences of the cardiac activity during VF. It is possible to create a single DFM from an optical mapping video using the full length of the time series. However, it is also possible to use short-time fourier transform, i.e. to apply the fourier transform to short segments of the video, in order to get a series of DFMs, each corresponding to some time window of the original video. As mentioned above, short fourier transforms will be used with a window length of $1 \mathrm{~s}$.

For each DFM we can calculate the mean dominant frequency which will be denoted in the following by ${\overline{f_{d}}}^{o p t}$. This mean will be determined more accurately the more pixels are used in the DFM.

Figure 4.6 shows exemplary DFMs for different time intervals of a recording of a rabbit heart during unperturbed VF with (upper row) and during pacing (lower row). This example illustrates on the one hand that the spatial distribution of dominant frequencies during VF is heterogeneous and that it changes over time. In fig. 4.6, histograms depict the number of pixels with certain frequencies below the single DFMs. The width of this distribution varies considerably over time. The DFMs and the histograms in the second row of fig. 4.6 illustrate how the pacing sequence causes a synchronization of the tissue such that essentially only one frequency remains that is equal to the pacing frequency (see third panel in the second row of fig. 4.6).

Some pixels have very low dominant frequency values (violet color) in some or all of the depicted DFMs. These values are typically caused by a low quality of the optical signal and it is unlikely that the heart tissue is actually activated with such low rates at those locations. However, also unexcitable tissue, e.g. scar tissue, can lead to a signal that has a very low dominant frequency.

\subsubsection{The Role of the Dominant Frequency}

In Section 4.1.1, I presented that the maximum activated area is a good indication whether a periodic pulse sequence leads to the termination of VF or not. Furthermore, I demonstrated that the effect of a periodic pulse sequence depends on its voltage and that the effect of the pulse sequence does not depend on the absolute pacing frequency $f_{p}$. The only exception is that in the data recorded from pig hearts the maximum activated area values drop above some upper frequency limit (see fig. 4.4). Using the tools of the previous section 4.1.4, I will now relate the effect of the pacing to the mean dominant frequency of the $\mathrm{VF} \overline{f_{d}}$ opt in order to see whether the effect of the periodic pulses depends on the dynamics in the heart. 
4.1 Control by Periodic Pulse Sequences

This page was intentionally left blank. 


\section{Results}

Figure 4.7 shows how the maximum activated area depends the voltage of the pulse sequence and on the frequency ratio $f_{p} / \overline{f_{d}}$ opt . Here, $f_{p}$ is the frequency of the pulse sequence and $\bar{f}_{d}^{o p t}$ is the dominant frequency of the optical signal before the pulses, i.e. before the block of the Synchronization Map. The control of the frequency ratio $f_{p} / \bar{f}_{d}^{o p t}$ is limited in the experiments. The experimenters observe the power spectrum of the optical signal, estimate the dominant frequency and set the cycle length of the pulse sequence accordingly in order to get some frequency ratio $f_{p} / \overline{f_{d}}$ opt . This visual estimation of the dominant frequency is error prone and additionally the dominant frequency is changing over time. Also, the range of used voltages was changed. Thus the data points in Figure 4.7 do not lie on a regular grid but are unstructured. The distribution of data points is depicted in the histograms on the right with a logarithmic color scale. For each point in the voltage-frequency-ratio-plane the mean value of data points in a surrounding disc is depicted. An exemplary disc is depicted in the lower left corners to illustrate size and shape. If less than 25 data points contribute to a mean value, no value is depicted in the diagrams on the left.

Figure 4.7 shows that the maximum activated area does not only depend on the voltage of the pulse sequence, but also on the frequency ratio. Interestingly, at a frequency ratio $f_{p} /{\overline{f_{d}}}^{o p t} \approx 1.1$ the maximum activated area increases at smaller voltages compared to other ratios and also the maximum value is reached at smaller voltages. The more the frequency ratio differs from 1.1 the more the levels of maximum activated area are shifted towards larger voltages.

Both datasets, the data from experiments with pig and rabbit hearts, show the overall same qualitative structure: While the frequency ratio $f_{p} /{\overline{f_{d}}}^{\text {opt }}$ does not seem to influence the maximum activated area for voltages below some value, the highest maximum activated area values are reached near a frequency ratio $f_{p} / \bar{f}_{d}{ }^{o p t} \approx 1.1$ for larger voltages.

In section 4.1.1 we found that a defibrillation by the pulse sequences are only probable when the maximum activated area values are near 1 . Thus, fig. 4.7 illustrates that defibrillations will (in the considered range of voltages) be more frequent near a frequency ratio $f_{p} /{\overline{f_{d}}}^{\text {opt }} \approx 1.1$.

In the data from pig and rabbit experiments, pulse sequences with voltages below $\approx 35 \mathrm{~V}$ and $\approx 20 \mathrm{~V}$, respectively, do not visibly increase the maximum activated area (see fig. 4.7). Does this imply, that these pulse sequence do not influence the dynamics at all? In the following section, I will show how periodic pulse sequence can change the dynamics of VF even though they do not lead to a large simultaneous activation and thus do not cause large values of the maximum activated area.

\subsubsection{Synchronization of Ventricular Fibrillation}

The synchronization that is described in $[55,44]$ and in the previous section is phase synchronization: Basically the whole tissue does locally the same oscillations in the phase space. However, in my numerical work of chapter 2 for example far field pulses were used to control the system and the frequency of excitation was uniform in the 
(a)

maximum activated area $\begin{array}{lllllll}0.60 & 0.65 & 0.70 & 0.75 & 0.80 & 0.85 & 0.90\end{array}$

(b) $\quad \mathrm{N}$ samples

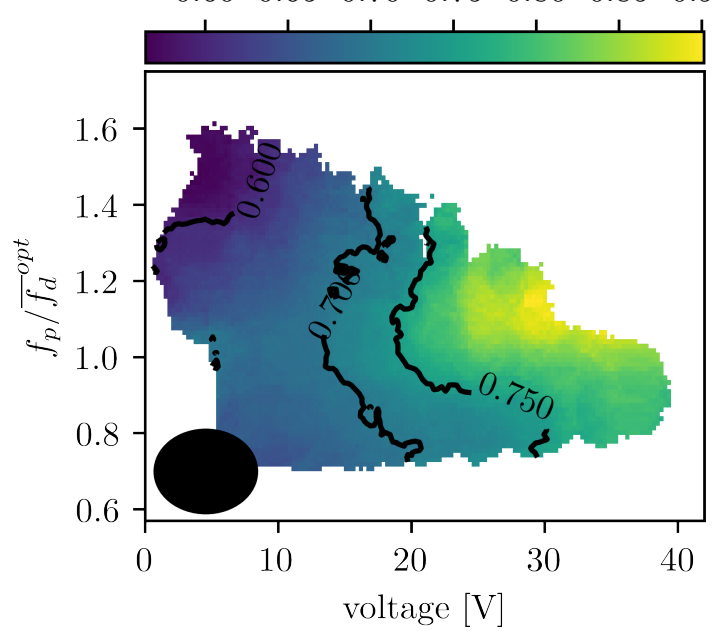

(c)

maximum activated area

$\begin{array}{lllllll}0.55 & 0.60 & 0.65 & 0.70 & 0.75 & 0.80 & 0.85\end{array}$
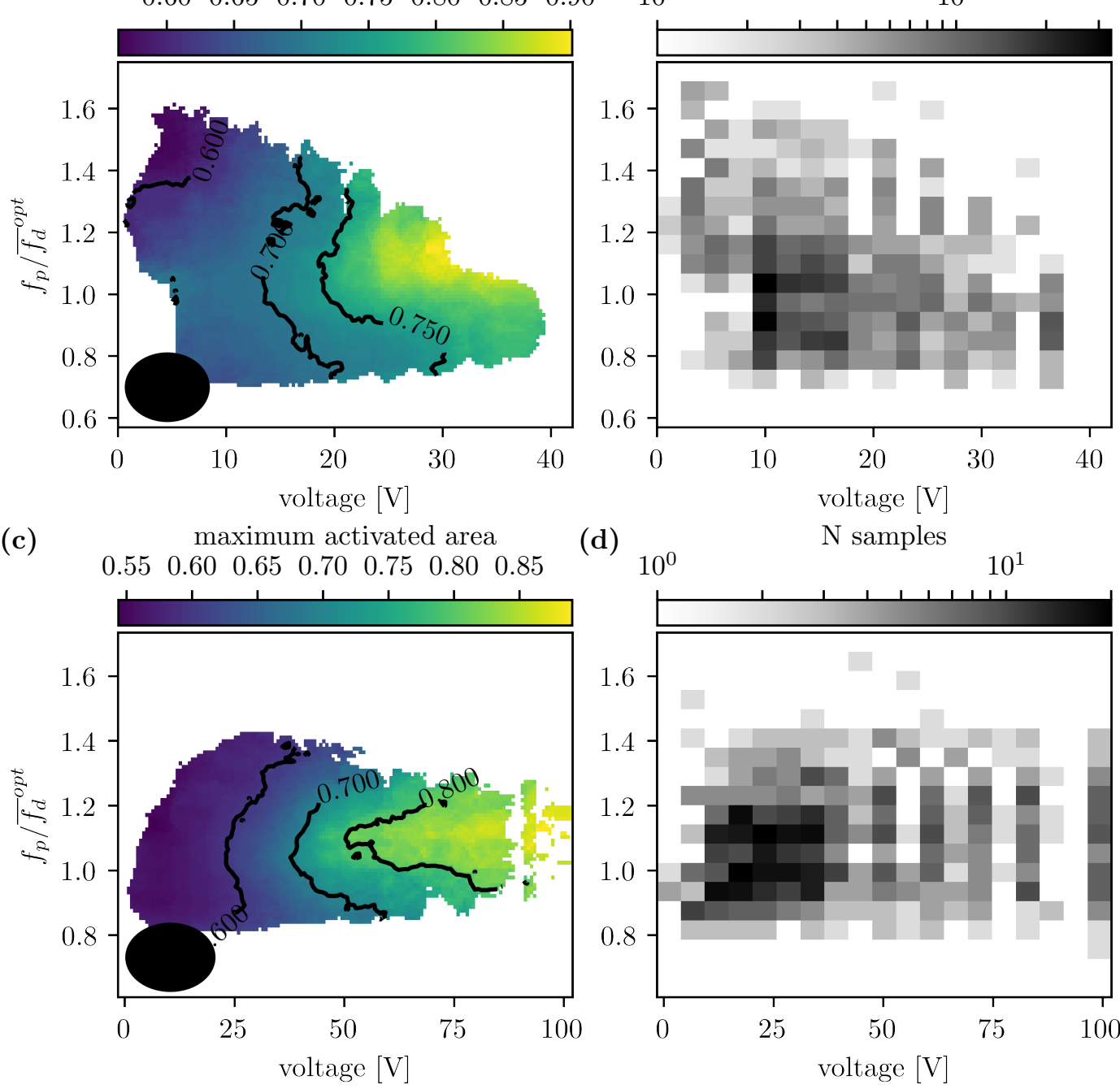

(d)

$\mathrm{N}$ samples

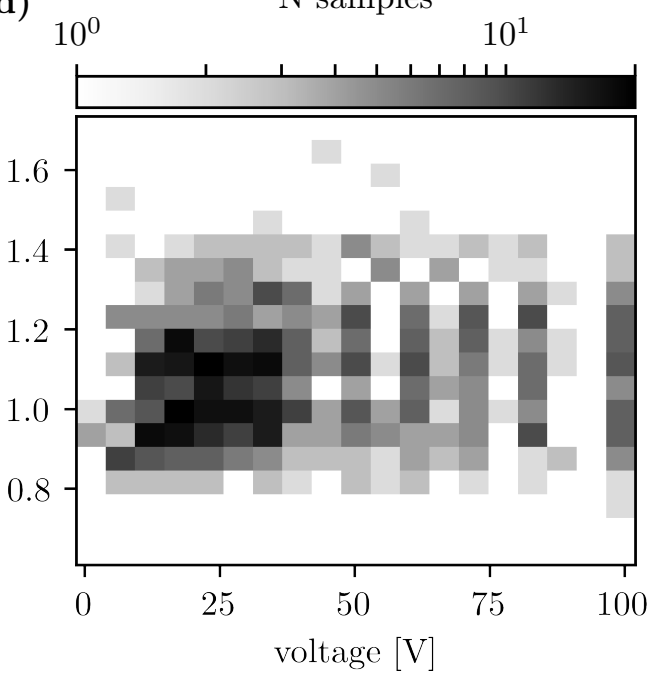

Figure 4.7: These diagrams present the dependence of the maximum activated area on the voltage of the pulses and on the ratio of the pacing frequency $f_{p}$ and the mean dominant frequency $\overline{f_{d}}$ opt . The unstructured distribution of data points is depicted on the right in 2D-histograms with a logarithmic color map. On the left, the maximum activated area is depicted color coded with the voltage and the frequency ratio on the axes. The diagrams (a) and (b) display the rabbit, diagrams (c) and (d) the pig dataset. At each point in the displayed parameter space the average of all data points that lie in a surrounding disc is depicted. The disc is displayed in the lower left in black. If less than 25 data points contribute to a mean value, no value is displayed. Generally, the maximum activated area increase with the voltage. However, for large voltages a dependency on the frequency ratio emerges, that is more clearly visible in the pig data. Here, the maximum activated area is increased near the frequency ratio of 1.1. 


\section{Results}

medium in the synchronized state, but the phases were not. Thus, I will investigate frequency synchronization caused by periodic pulses in the following. How periodic pulses can synchronize the dynamics of VF in the sense that the heart tissue is every where excited with the same frequency as the pacing frequency, but not necessarily at the same time.

In order to quantify the synchronization, I measure the fraction of the heart surface that has a dominant frequency matching the frequency of the pacing. This measure will be called synchronized area fraction in the following. Refer to the lower row of fig. 4.6 for an example where the pulse sequence leads to a spatially homogeneous distribution of the dominant frequency. Almost the whole tissue is excited with a frequency that equals the pacing frequency, i.e. the synchronized area fraction in the last frame is close to 1 .

Please note, that this measure differs significantly from the maximum activated area used in the previous section as a full frequency synchronization can for example be achieved by pulses that cause planar waves that travel over the whole muscle. In this case, the whole tissue would not be excited at once, but with the same frequency. We will thus look at a different kind of control in this section compared to the previous one.

Figure 4.8 shows the mean synchronized area fraction in the last eighth of a pulse sequence depending on the frequency ratio $f_{p} /{\overline{f_{d}}}^{\text {opt }}$ and the voltage of the pulses. The figure is structured as the corresponding ones before: On the right the unstructured distribution of data points is depicted with a logarithmic color scale. The top row and bottom row show data from experiments with rabbit and pig hearts, respectively. Due to variations of the synchronized area fraction for very similar parameter values, the average value of the data points in a disc centered at the respective location is displayed. The disc is shown in the lower left of the diagrams.

The main qualitative features are similar to those found in the corresponding plots with the maximum activated area as a measure: The synchronized area fraction increases with the voltage and this increase occurs at smaller voltages the closer the frequency ratio is to 1.1. However, while there was no clear frequency dependency below $\approx 35 \mathrm{~V}$ in the pig data and below $\approx 20 \mathrm{~V}$ in the rabbit data, fig. 4.8 shows larger synchronized area fractions around 1.1 also in this range of low voltages.

Figure 4.7 illustrates for which voltages and frequency ratios $f_{p} / \overline{f_{d}}$ opt a periodic pulse sequence has a high chance to change the dynamics drastically, such that large fractions of the heart surface are activated simultaneously. In contrast, fig. 4.8 demonstrates, how the dynamics are changed by periodic pulse sequences in terms of the frequency with which the tissue is activated. In most areas of the parameter space that is shown in fig. 4.8, the structure is strikingly simple and very similar for both data from experiments with rabbit and pig hearts: Contour lines form wedges made of straight lines with the tip being between a frequency ratio of 1.0 and 1.2.

Figure 4.8 consists of data from many experiments. However the same qualitative features can be found in single experiments as figs. 4.9 and 4.10 illustrate. The data of single experiments is difficult to interpret as only very few data points exist. Still, 
(a)

synchronized area fraction
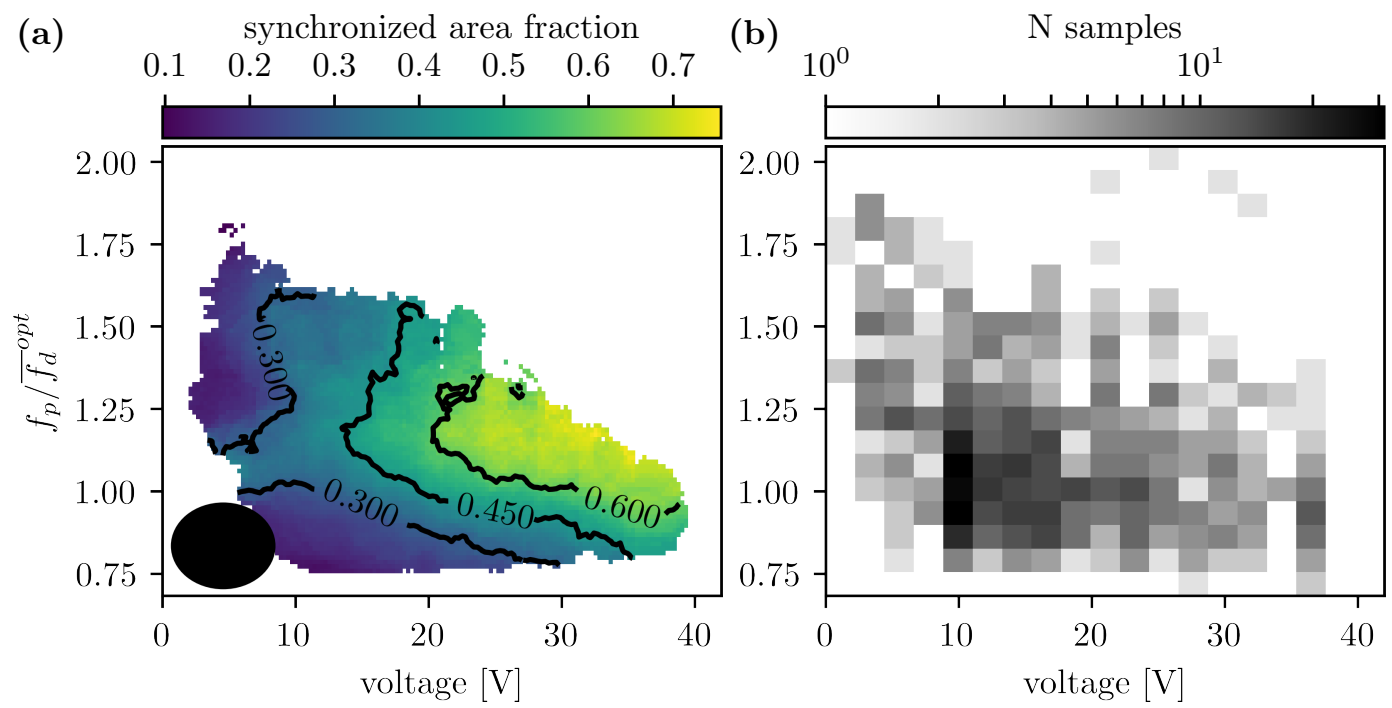

(c)

synchronized area fraction $\begin{array}{lllllll}0.2 & 0.3 & 0.4 & 0.5 & 0.6 & 0.7 & 0.8\end{array}$

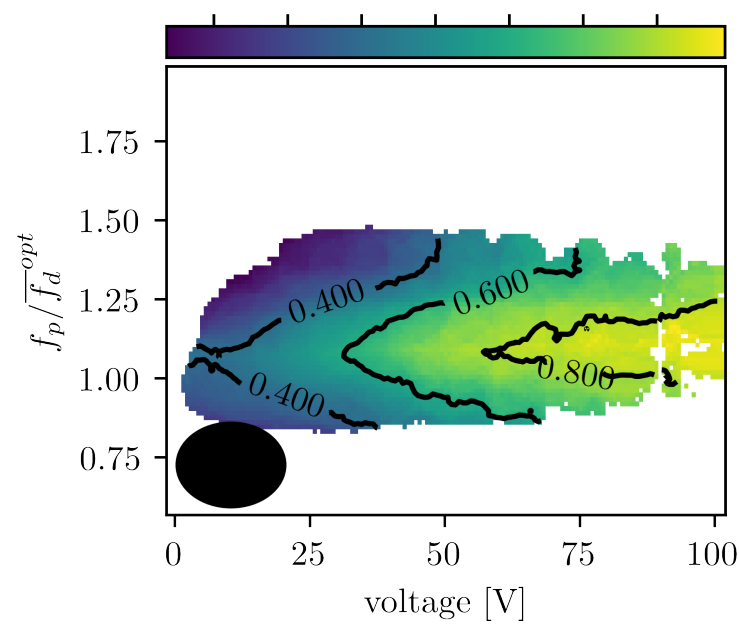

(d)

$\mathrm{N}$ samples

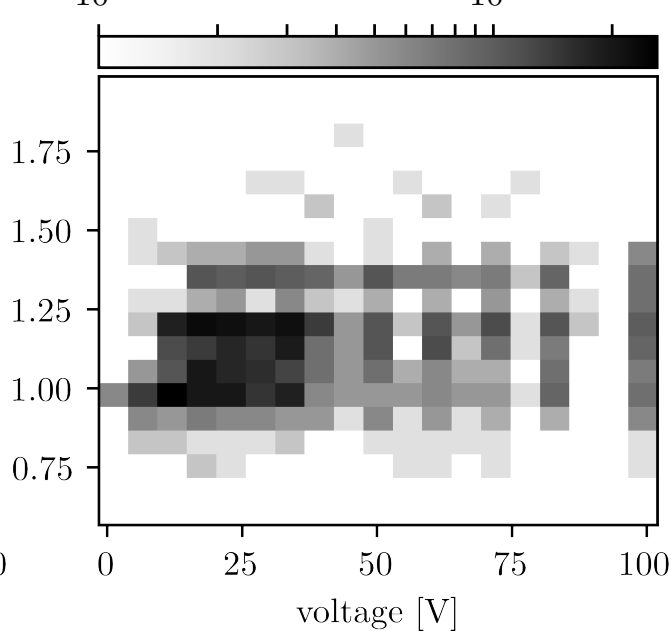

Figure 4.8: These diagrams present the dependence of the synchronized area fraction on the voltage of the pulses and on the ratio of the pacing frequency $f_{p}$ and the mean dominant frequency $\overline{f_{d}}$ opt . The unstructured distribution of data points is depicted on the right in 2D-histograms with a logarithmic color map. On the left, the synchronized area fraction averaged over the last eighth of the pacing is depicted color coded with the voltage and the frequency ratio on the axes. The diagrams (a) and (b) display the rabbit, diagrams (c) and (d) the pig dataset. In the left diagrams, the depicted value is the mean of data points in a surrounding disc with a size that is displayed in the lower left in black. If less than 25 contribute to a mean value, no value is displayed. Generally, the synchronized area fraction increase with the voltage. Additionally, there is a strong dependency on the frequency ratio. The synchronized area fraction is the largest near the frequency ratio $\approx 1.1$. 


\section{Results}

most diagrams for single experiments seem to be in agreement with the structure shown in fig. 4.8. These 30 diagrams are not included in this thesis but can be viewed online [76].

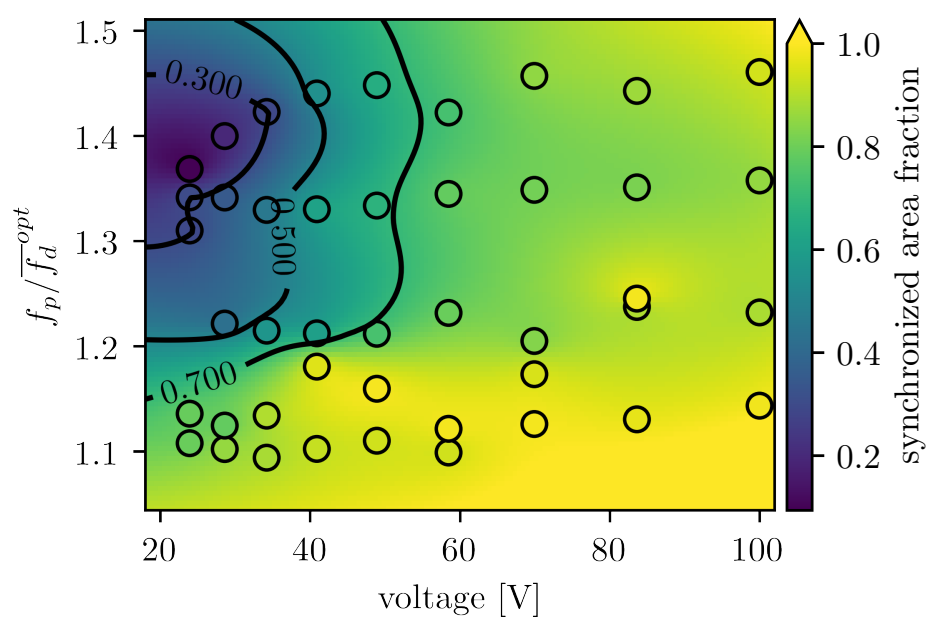

Figure 4.9: The synchronized area fraction that is caused by periodic pulse sequences is shown depending on the voltage of the pulses and on the frequency ratio $f_{p} / \overline{f_{d}}$. The data points shown as circles are the actual recordings, while the remaining colored area is created from an interpolation with a $1 / r$ radial function, with $r$ being the distance of two points in the diagram. The depicted data is from an experiment with a pig heart on 15 Feb 2018. The animal model is a chronic heart failure model. The pulses were applied using two plate electrodes.

Due to the clear structure in fig. 4.8 , it might be tempting to expect a deterministic behavior for the synchronization, i.e. that a certain parameter combination always leads to a specific synchronized area fraction. However, due to the stochastic nature of $\mathrm{VF}$, values can vary considerably for single recordings. This is illustraded by fig. A.5 which is analogous to fig. 4.8, except that nearest neighbor interpolation is used instead of averaging. Figure A.5 on page 107 depicts the same data but with nearest neighbor interpolation instead of averaging.

Together, Figures 4.7 and 4.8 allow to make predictions, what kind of control can be expected for pulse sequences depending on the ratio $f_{p} / \bar{f}_{d}{ }^{o p t}$ and the voltage of the pulses. Especially, they make the prediction that a pulse sequence with a frequency ratio $f_{p} /{\overline{f_{d}}}^{\text {opt }} \approx 1.1$ will have the largest effect, both in terms of frequency synchronization and activation.

\subsubsection{Successive Synchronization during Periodic Pulses}

So far, I quantified the control that exists in the end of a pulse sequences. Let us now consider the temporal change of the dynamics during the control with periodic 


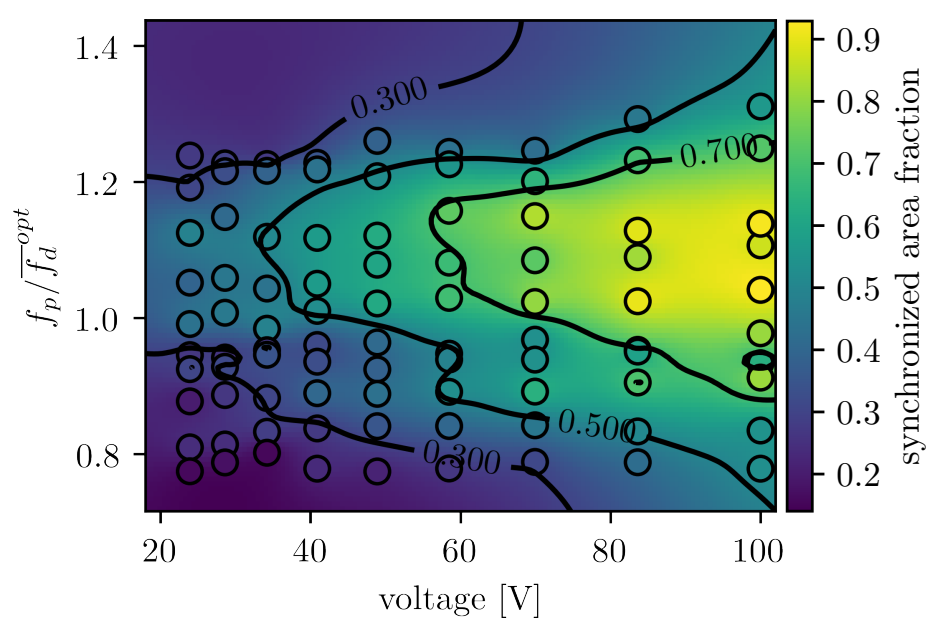

Figure 4.10: The synchronized area fraction that is caused by periodic pulse sequences is shown depending on the voltage of the pulses and on the frequency ratio $f_{p} / \overline{f_{d}}$. The data points shown as circles are the actual recordings, while the remaining colored area is created from an interpolation with a $1 / r$ radial function, with $r$ being the distance of two points in the diagram. The depicted data is from an experiment with a pig heart on 23 May 2018. The animal model is a chronic myocardial infarction model. The pulses were applied using two plate electrodes or a plate electrode and a catheter. The different electrode configuration might be the reason for the slightly, horizontally striped appearance of diagram. Still, the overall structure seems to be unchanged by the use of different electrodes configurations.

pulses. This will provide information on how many pulses are necessary in a periodic sequence to reach the maximum control.

The synchronized area fraction during the application of four Synchronization Maps to a pig heart in VF is shown in fig. 4.11. Each curve is a single recording corresponding to one block of the Synchronization Map, i.e. a pulse sequence with a single voltage. These single traces are grouped into five diagrams according to the respective voltage which is indicated in the upper left corner. The first pulse of the sequences is applied where the time equals zero. Thus time values that are smaller than zero correspond to unperturbed $\mathrm{VF}^{6}$. Note, that the Fourier transform was applied to windows with a length of $1 \mathrm{~s}$ to calculate the synchronized area fraction. Thus, a value depicted at time $t$ is an average for the time window $[t-1 \mathrm{~s}, t]$. The curves are colored according to the frequency ratio $f_{p} / \bar{f}_{d}^{o p t}$ of the respective pulse sequence.

Generally, the higher the voltage the larger is the increase of the synchronized area fraction. The increase is also steeper for higher voltages. In the diagram corresponding to the lowest voltages mostly random fluctuations of the synchronized area fraction can

\footnotetext{
${ }^{6}$ The gaps between blocks are typically of $4 \mathrm{~s}$ duration. Thus previous blocks might influence the VF even after this time. In this sense, the dynamics before all blocks but the first are not unperturbed.
} 

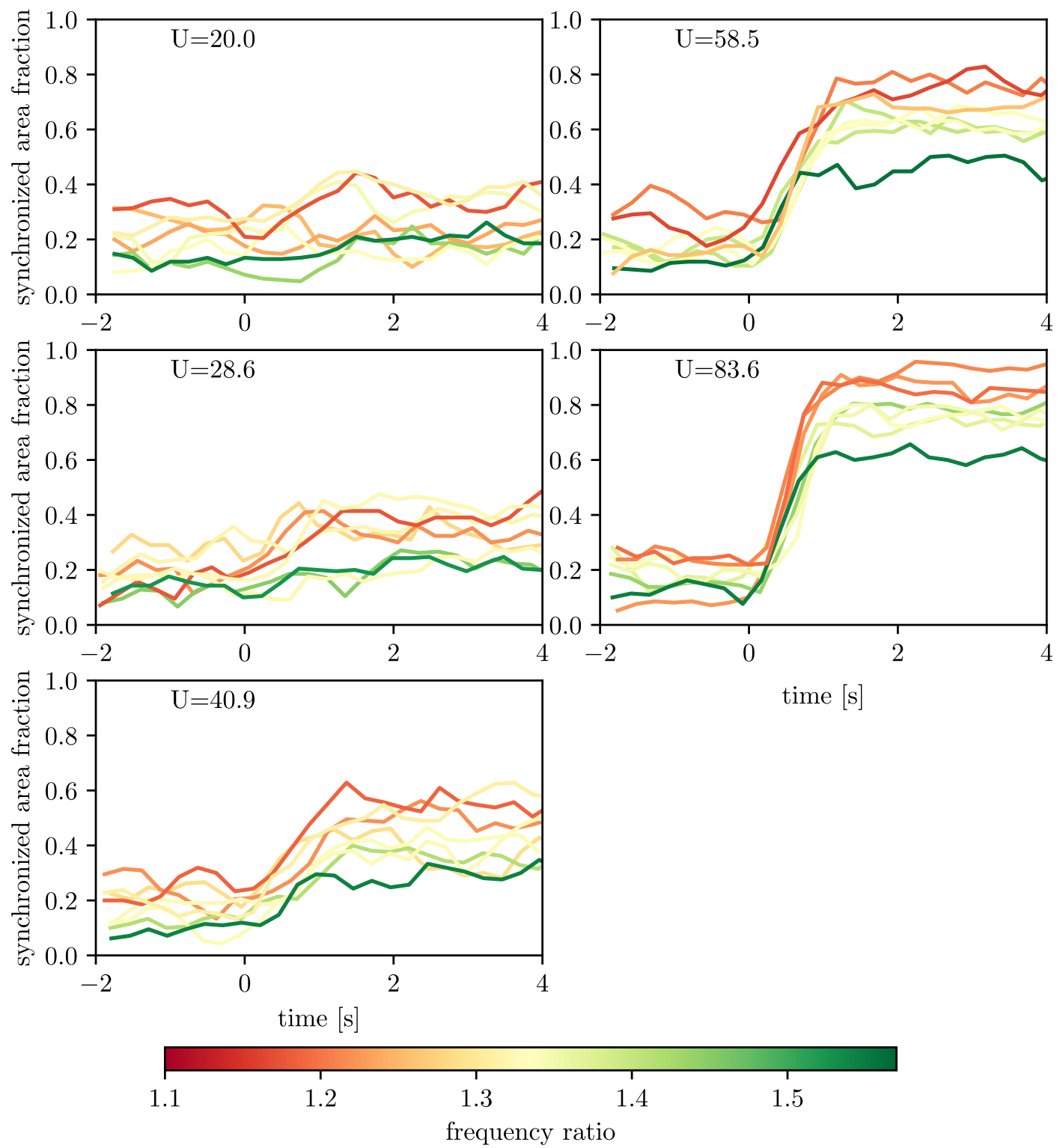

Figure 4.11: The increase of the synchronized area fraction over time during periodic stimulation of a pig heart during VF is shown. Each curve is a single recording corresponding to one sequence of pulses with a single voltage. The curves are grouped according to their voltage (indicated in the upper left corner). The single curves are colored according to the frequency ratio $f_{p} / \overline{f_{d}}$ opt of that pulse sequence. The higher the voltage is the larger is the increase of the synchronized area fraction. The synchronized area fraction is tendentially larger for frequency ratios close to 1.1. 

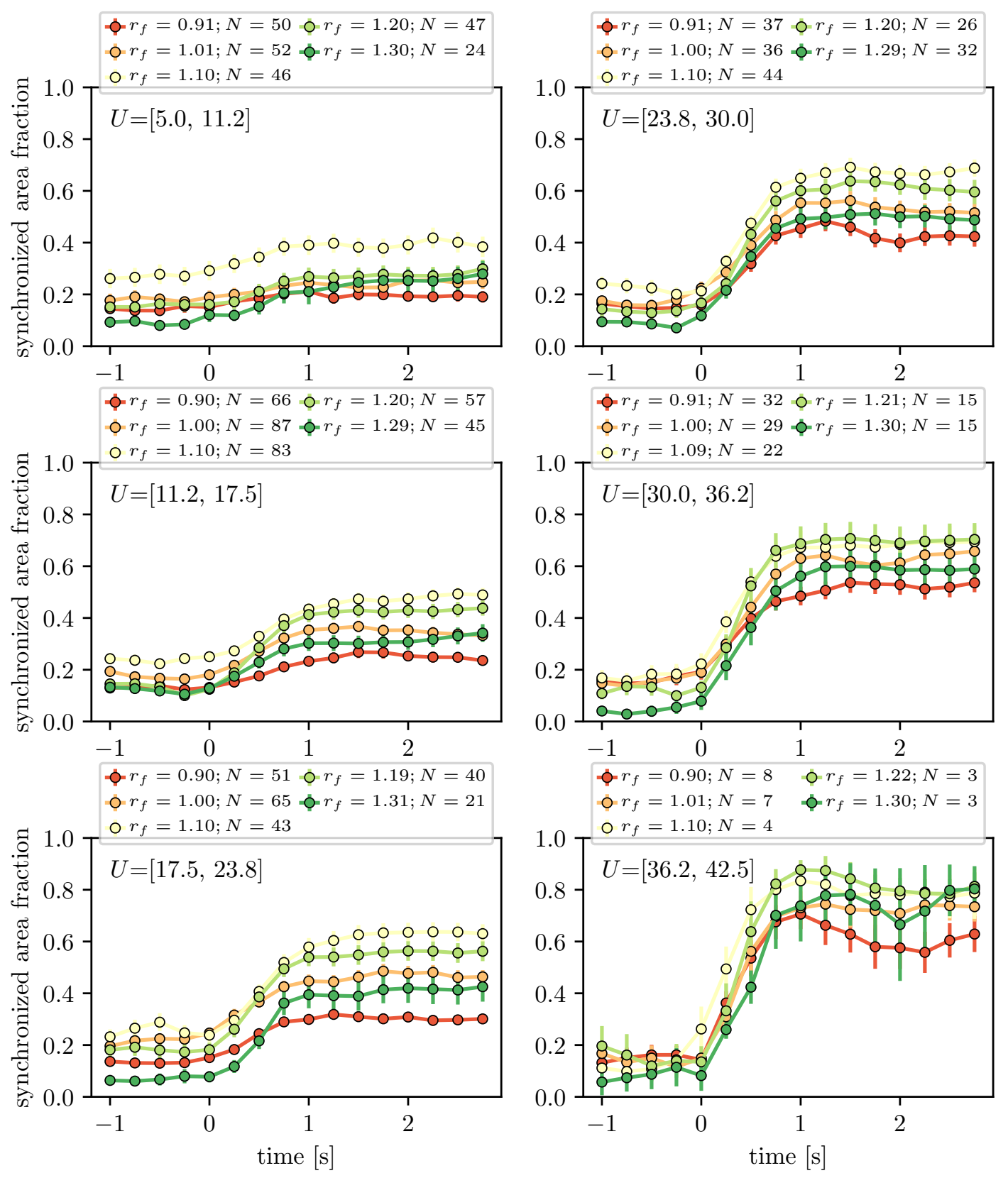

Figure 4.12: Increase of the synchronized area fraction over time during periodic stimulation of rabbit hearts in VF. Each curve is the average of $N$ recordings that lie in respective intervals of the voltage $U$ (denoted in the upper left corner) and the frequency ratio $r_{f}=f_{p} /{\overline{f_{d}}}^{\text {opt }}$ (mean given in the legend). Errorbars depict the standard error of the mean. First pulse is applied at time 0 . The increase of the synchronized area fraction is steeper for higher voltages. The highest values occur for frequency ratios $r_{f}$ near 1.1. Already after $1 \mathrm{~s}$ to $2 \mathrm{~s}$ a plateau is reached in all cases. 

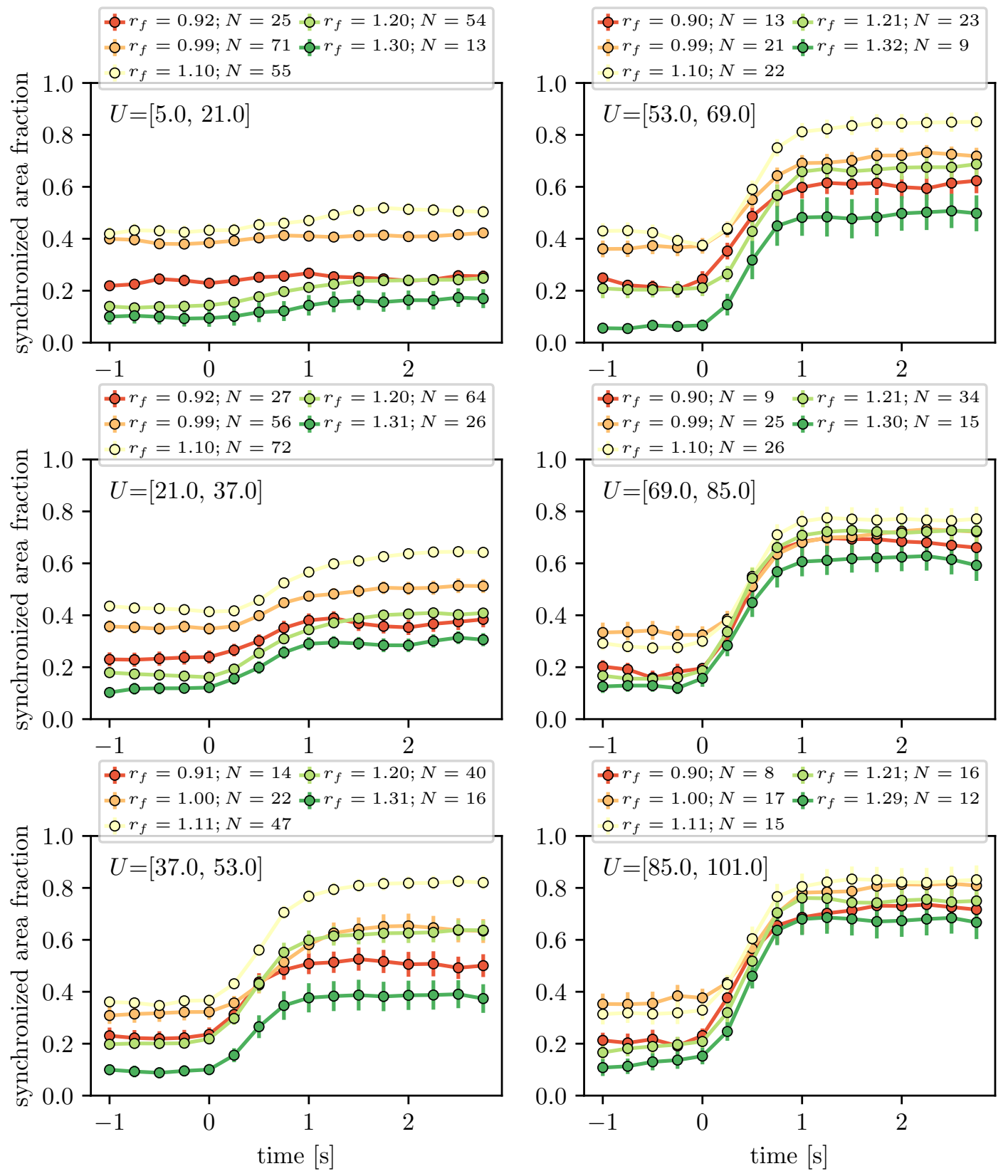

Figure 4.13: Identical to fig. 4.11 but with data recorded from pig hearts. Increase of the synchronized area fraction over time during periodic stimulation. Each curve is the average of $N$ recordings that lie in respective intervals of the voltage $U$ (denoted in the upper left corner) and the frequency ratio $r_{f}=f_{p} / \bar{f}_{d}^{\text {opt }}$ (mean given in the legend). Errorbars depict the standard error of the mean. First pulse is applied at time 0 . The increase of the synchronized area fraction is steeper for higher voltages. The highest values occur for frequency ratios $r_{f}$ near 1.1. Already after $1 \mathrm{~s}$ to $25 \mathrm{~s}$ a plateau is reached in all cases. 
be seen. Also in the other diagrams fluctuations of the synchronized area fraction are visible although the synchronized area fraction is fairly constant in the single curves once the maximum value is reached. The synchronized area fraction is tendentially larger for frequency ratios close to 1.1 (here colored in red).

The curves seem to be ordered even before the onset of the pulses $(t<0)$. On one hand this might be due to a remaining effect of preceding blocks in a Synchronization Map. However, it is likely that this is mostly due to the fact that there is a spatial distribution of dominant frequencies during VF (see section 4.1.3). Assuming the distribution is mono modal as in fig. 4.6, the synchronized area fraction during unperturbed VF will be larger when the reference frequence ("pacing frequency") is near the peak of the distribution. Also, the mean dominant frequency ${\overline{f_{d}}}^{\text {opt }}$ considered here is typically close to this peak. Therefore time series will have a larger synchronized area fraction in the part before the pacing the closer their frequency ratio $f_{p} / \bar{f}_{d}^{\text {opt }}$ is to 1 .

In order to check whether the above example is representative, I analyzed the average increase of the synchronized area fraction in all experiments. Figures 4.12 and 4.13 are designed analogously to fig. 4.11, but they show the data of all Synchronization Maps in the datasets $S y n c P$ and $S y n c R$ recorded from pig and rabbit hearts, respectively. The voltages and frequency ratios of all blocks of the recorded Synchronization Maps are binned. Each curve is the average of all time series of individual blocks of Synchronization Maps that lie in the corresponding voltage and frequency ratio bin. The voltage interval that is used for a specific plot is indicated in the upper left corner while the frequency ratio is color coded. Errorbars depict the standard error of the mean. In the last voltage bin, there are fewer recordings than in the others and the error of the mean is therefore larger.

The qualitative features of the graphs in figs. 4.12 and 4.13 are the same as in fig. 4.11 where the data from a single experiment was shown. The increase of the synchronized area fraction is larger and steeper for higher voltages. The curves corresponding to a frequency ratio bin of 1.1 have always the largest or second largest synchronized area fraction values.

Furthermore, already after $1 \mathrm{~s}$ to $1.5 \mathrm{~s}$ most curves reach a plateau extending to the end of the time series. Even if the effect of the periodic shocks would be immediate, i.e. the first shock synchronizes the tissue and the shocks thereafter do not change anything, the transition would be a linear increase over $1 \mathrm{~s}$ due to the time averaging. Such a fast transition might be expected for the high voltages were the first pulse changes the dynamics drastically and quasi instantly synchronizes the tissue (for those high voltages the maximum activated area was very large in fig. 4.7). But for smaller voltages this implies that the pulses have no combined value, i.e. each pulse only has the effect of its predecessor. Consider for example the lower left axis in fig. 4.12. The red curve corresponding to a frequency ratio $f_{p} / \bar{f}_{d}$ opt of about 0.8 does not considerably increase after $t=1 \mathrm{~s}$. The yellow curve corresponding to a frequency ratio of around 1.1 increases until $t=1.5 \mathrm{~s}$. I mentioned before that in excitable media, faster activity can push away slower activity. Thus this difference of the increase can 


\section{Results}

be interpreted as follows: The slow pacing can only control the tissue where excitation is directly caused, while the faster pacing successively recruits surrounding tissue.

With an increase of the voltage the hight of the plateaus of the different frequency ratios spreads apart and then converges again (except for the highest voltages in the data from experiments with rabbit hearts). This is an indication that while a comparably large synchronized area fraction for $t<0 \mathrm{~s}$ corresponds to the frequency ratio of 1.1, this frequency ratio increases the the synchronized area fraction further, i.e. the additionally recruited area is larger for this ratio than for the others.

While the values in the plateau are quite constant for most curves, some curves in the diagram with the largest voltages of fig. 4.12 drop again after they reached the maximum. While the error of those data points is comparably large, the drop is of a similar magnitude as the error. It is thus unclear whether the synchronized area does on average decline again after it reached the maximum in recordings with sequences of the highest voltages.

\subsubsection{Section Summary}

Initially, I presented experimental evidence that high values of the maximum activated area coincide with a high probability that a sequence of periodic pulses terminates VF. Afterwards, I investigated how parameters of such pulse sequence influence the maximum activated area. While this quantity is positively correlated with the voltage of the pulses, the absolute frequency has little influence on the maximum activated area. Only if a pacing frequency larger than $8 \mathrm{~Hz}$ is chosen in experiments with pig hearts, the maximum activated area will be smaller than for smaller frequencies. In contrast, the maximum activated area clearly depends on the frequency ratio $f_{p} / \overline{f_{d}}$ opt and shows the largest values for a ratio around 1.1.

Furthermore, I introduced the synchronized area fraction, a measure for the frequency synchronization. I demonstrated that the synchronized area fraction also clearly depends on the frequency $f_{p} / \bar{f}_{d}^{\text {opt }}$ and takes different values even for comparably low voltages where the maximum activated area was nearly the same for all frequency ratios.

In the last part, I demonstrated how the synchronized area fraction increases during the application of periodic pulse sequences. After the onset of the pulse sequence the synchronized area fraction increases until a plateau is reached. Even though in the analysis the values are averaged over a $1 \mathrm{~s}$ time window the transition is typically shorter than $2 \mathrm{~s}$.

These findings are especially valuable when designing pulse sequences that shall exercise a maximum control. In particular it provides information about the frequency ratio, the voltage and the number of pulses that should be chosen to achieve the desired synchronization. 


\subsection{Synchronization Preceding Termination of VF}

In chapter 2, I presented an efficient technique to control a system with multiple pinned spirals. Undoubtedly, the dynamics in the simulations of chapter 2 are very different from the dynamics during VF. For example, the spirals in the simulations create very periodic excitations while spiral waves in VF are often transient and the dynamics are overall much more irregular. Thus, as it was also pointed out in the manuscript, it cannot be expected that the results from the simulations can directly be transferred to the VF scenario. Nevertheless, the pinning of spiral waves is still discussed as a mechanism that sustains VF (see section 1.7) and many qualitative features like the existence of spiral waves and properties of excitable media are the same in the simulations and cardiac tissue during VF. Therefore, I decided to test experimentally whether an analogous control strategy is able to terminate VF efficiently. My colleagues and I designed a new defibrillation technique, where a sequence of pulses synchronizes the tissue and an additional pulse terminates the arrhythmia, based on the insights from the theoretical and numerical work of chapter 2 .

Due to the initial synchronization step, this new defibrillation technique can also be seen as an attempt to exploit the control by periodic pulses presented in the previous chapter. In other words: Does the more regular state of VF created by the periodic pulses promote the termination of VF using a single electric pulse?

Please note, that this approach differs from what was already discussed in the previous section, where I considered periodic pulses that directly lead to the termination of VF. Instead, I will now consider a combination of a periodic pulse sequence that creates an ordered and homogeneous state and an additional single shock that terminates the arrhythmia.

As mentioned before, high energies of electric far field shocks used for defibrillation are associated with pain and tissue damage. Thus, defibrillation techniques using electric far field shocks are typically compared in terms of the energy necessary for defibrillation. I will therefore compare the new defibrillation method with two other techniques, one being the conventional standard defibrillation. However, there are multiple characteristic energies that could be used for a comparison and even more techniques to calculate those. In the following section, I will therefore describe how the characteristic energy $E_{50}$, which will be used here, can be calculated and how its associated uncertainty can be estimated.

\subsubsection{Dose Response Curve}

Whether a defibrillation technique using electric far field shocks, like standard defibrillation, terminates VF or not depends on the energy of the used electric shocks. The defibrillation curve, also called dose response curve, describes this dependency. The logistic function

$$
\sigma(E)=\frac{1}{1+\exp \left(\beta_{0}+\beta_{1} E\right)}
$$




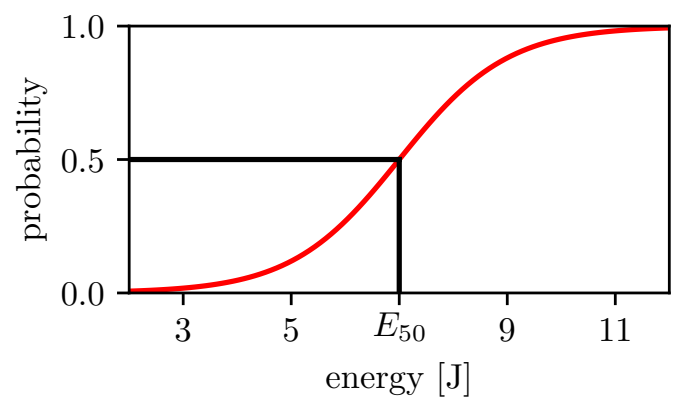

Figure 4.14: Example of a dose response curve with a logistic curve as model. The probability that a defibrillation attempt is successful is plotted versus the used energy. The energy $E_{50}$ at which a termination has $50 \%$ probability is indicated by black lines.

is a common model for the dose response curve and it was shown that it reasonably fits experimentally measured data consisting of single shock defibrillation attempts [24]. A characteristic value of this curve is the energy $E_{50}$ (see fig. 4.14) at which the chance for successful defibrillation is $50 \%$ and which is also sometimes called defibrillation threshold (DFT). The term DFT stems from the assumption that the transition of the dose response curve from never successful to always successful occurs abruptly. Rattes et al. [69] argue that the DFT concept is still used due to its simplicity even though an abrupt change of success probability is often not given. The concept of the dose response curve is used when investigating how defibrillation can be tested in patients in order to estimate the DFT and especially an energy that with very large probability terminates VF.

The concept of the dose response curve, or at least its characteristic $E_{50}$ value, is often estimated in experiments or studies to quantify or compare defibrillation techniques. As the dose response curve will also be employed in this thesis, I will describe in the following how this curve can be measured in experiments and what needs to be considered in the analysis.

It is difficult to measure the parameters of the dose response curve in experiments accurately because often only few defibrillation events are recorded. Each event gives a binary data point (termination of $\mathrm{VF}$ and no termination) that is then used to fit the model. An unfavorable distribution of measurements, e.g. only failed defibrillations, will lead to large uncertainties of parameter estimates or prevent estimates completely. Additionally, it is important to consider whether the dose response curve, i.e. both parameters, shall be estimated or if only the $E_{50}$ value shall be estimated. Because one goal is to provide a defibrillation method that uses a smaller energy, I will restrict the analysis to the estimation of $E_{50}$ in the following ${ }^{7}$. However, experimental procedures exist that reduce the number of measurements necessary for a good estimation of the

${ }^{7}$ Other energy levels would also be possible, i.e. $E_{90}$ where termination probability is $90 \%$, these however require more measurements for a comparable uncertainty. 
$E_{50}$ value.

An efficient method to measure a characteristic value like $E_{50}$ has been known for a long time from bioassay methods. In those methods, the responses (of e.g. animals) to certain dose levels (of e.g. a drug) are recorded. The dose levels can be determined using an up-down protocol in which the dose is increased after a response and decreased after a non-response. In 1965, Dixon [26] described how to improve the estimates of the dose level corresponding to $50 \%$ chance for a response and its uncertainty when using an up-down-protocol. In the context of defibrillation, McDaniel and Schuder [58] discussed how different $E_{50}$ estimation methods can be compared and suggested an up-down-protocol with four defibrillation attempts, however without the improvement of [26]. The methods used in the literature to estimate $E_{50}$ of different defibrillation techniques vary considerably $[20,56,82,45]$. This is partly because some authors assume a steep transition in the dose response curve and measure the DFT (see above). They thus use methods with rather few measurements.

In the experiments that contributed data to this thesis, we used an up-down-protocol with steps of about $20 \%$ energy. Thus if a defibrillation attempt fails, the next measurement occurs at 1.2 times the previous energy and if the defibrillation was successful, the energy is divided by 1.2. This has the advantage over absolute energy steps that the sampling always has an approximate resolution of $20 \%$ of the energy $E_{50}$ independent of the actual $E_{50}$ value.

In the following, I will demonstrate how recorded defibrillation attempts are analysed and how the uncertainty of $E_{50}$ can be estimated. Let us assume the dose response curve of some defibrillation technique is a logistic curve with the parameters $\beta_{0}=-5$ and $\beta_{1}=1$ as shown in fig. 4.15 in blue. The $E_{50}$ value can be related to these parameters via $\beta_{0}+\beta_{1} E=\left(E-E_{50}\right) / w$, with $w$ being the "width" of the transition of the curve. Thus, $E_{50}=-\beta_{0} / \beta_{1}=5$ and $w=1 / \beta_{1}=1$. An experiment will yield a success at a certain energy with the probability that is predicted by this model. We can thus simulate an experiment with $N$ observations analogous to the experimental protocol using the following algorithm:

1. Select an arbitrary start energy from the interval $\left[E_{50}-w, E_{50}+w\right]$ as current energy.

2. Simulate the outcome of a defibrillation attempt: randomly select successful or unsuccessful based on the success probabilities given by the model according to the current energy.

3. Increase or decrease the current energy according to the up-down-protocol.

4. If less than $N$ defibrillation attempts were simulated, continue with 2 ., otherwise end.

An exemplary dataset with 50 observations created with this algorithm is depicted in fig. 4.15. Successful defibrillations are shown as ones and failures as zeros. The red curve shows the logistic curve that was fitted to this data using logistic regression. This fit has a slightly different $E_{50}$ and a different $w$ which can be expected. 


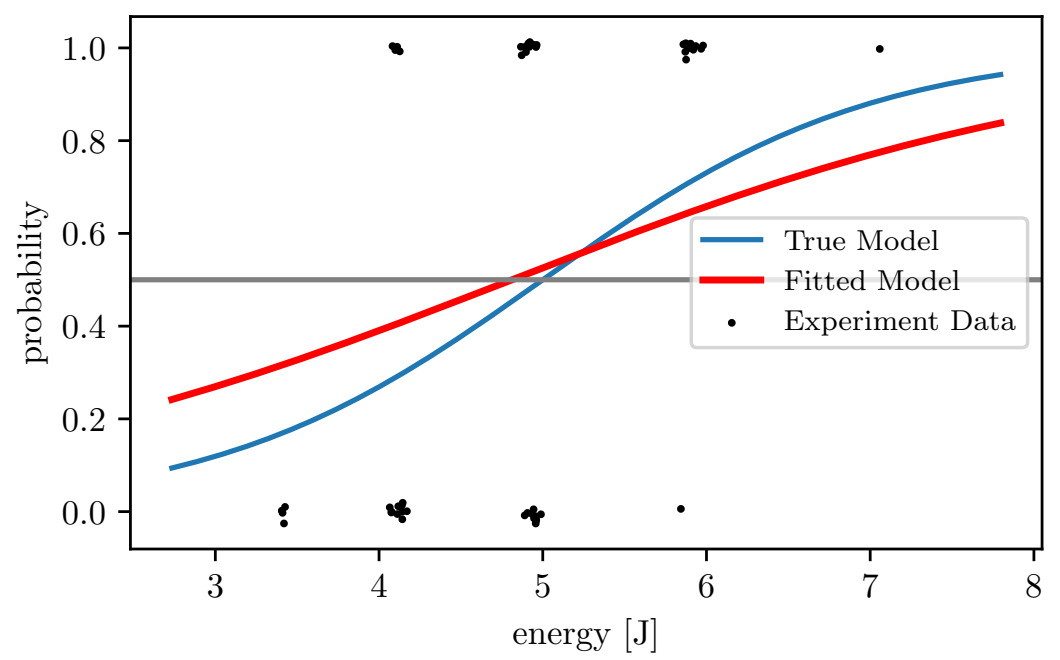

Figure 4.15: Success probabilities of defibrillation attempts are plotted versus the used energy. A logistic model is assumed to be the true model (blue) for a simulated experiment. The resulting data ( 50 attempts) is depicted by black dots with an artificial jitter; zeros for failures, ones for successes. The data is fitted with the red curve using logistic regression. The fit has a different slope and $E_{50}$ compared to the true model.

The fitted parameter $\beta_{0}=-8.7$ has a confidence interval of $[-14.0,-3.5]$. If we neglect the contribution of the uncertainty of $\beta_{1}$, we can estimate the confidence interval of $E_{50}$ by multiplying the confidence interval for $\beta_{0}$ with $-\beta_{1}$. This gives a confidence interval for $E_{50}$ of $[1.9,7.7]$. However, when we simulate many more of such experiments, each starting at some random energy uniformly distributed among [5-w, $5+w]$, and compute the $E_{50}$ value for each we get a distribution as shown in fig. 4.16. In statistical terms, the defibrillation attempts constitute a random sample $\mathbf{X}=\left(X_{1}, X_{2}, \cdots X_{N}\right)$ of size $\mathrm{N}$ and an observed realization $\mathbf{x}=\left(x_{1}, x_{2}, \cdots x_{N}\right)$ is depicted in fig. 4.15. The $E_{50}$ calculation from a sample using the logistic regression can be seen as a statistic and fig. 4.16 shows the (approximate) sampling distribution. In the following analyses, 5000 experiments will be simulated in order to get estimates of the sampling distribution. The standard error and the confidence interval of the statistic can be directly calculated from this distribution. As indicated in fig. 4.16, the $95 \%$ confidence interval is roughly $[4.5,5.5]$ and thus much smaller than the one derived from the logistic regression even though the uncertainty contribution of $\beta_{1}$ to the latter was neglected.

This example illustrates that the uncertainties from the logistic regression can greatly overestimate the real uncertainties. Thus, I will use a different approach to estimate the uncertainties of $E_{50}$ values based on the above described procedure using simulated experiments. This method was described in the context of Monte Carlo Intervals and bootstrap methods $[28,14]$. The key idea is that an observed realization $\mathbf{x}$ is used to estimate the parameters of the underlying model which allows to simulate many realizations $\mathbf{x}_{i}^{\prime}$ and to generate a sample distribution. This procedure basically restricts 


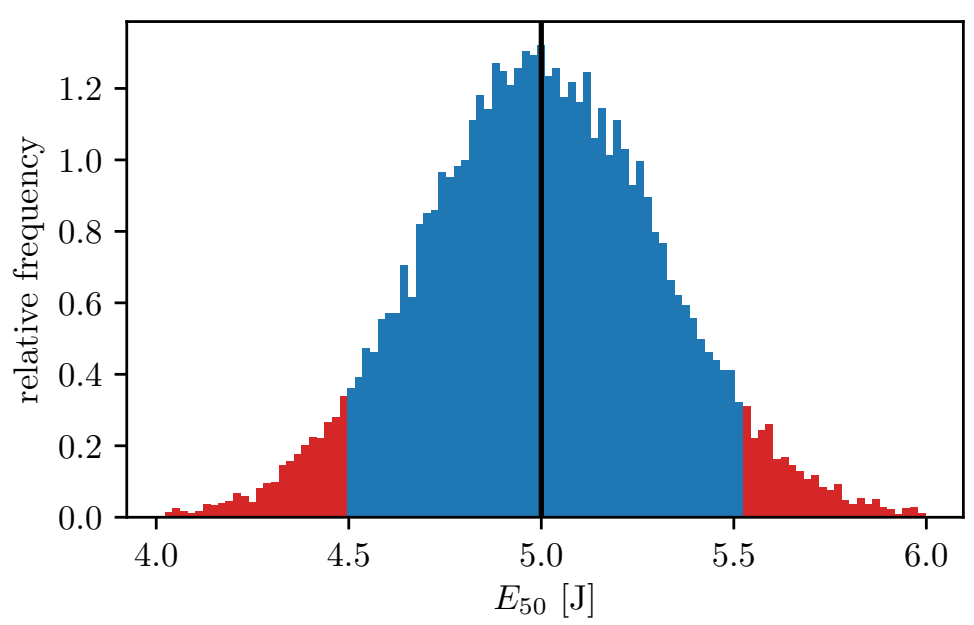

Figure 4.16: Normalized histogram of calculated $E_{50}$ values from 20,000 simulated experiments. The mean calculated $E_{50}$ equals the true $E_{50}$ of 5 . Roughly, $95 \%$ of the values lie in the interval $[4.5,5.5]$ indicated in blue.

the range of possible defibrillation curves and thereby also reduces the uncertainty.

To summarize the above said, I will assume that the observed realization $\mathbf{x}$ of defibrillation attempts in an experiment allows to approximate the dose response curve of the corresponding defibrillation technique. This model is then used to simulate experiments and generate observations of the same size and thus create the sampling distribution. From the sampling distribution the standard error and confidence intervals of $E_{50}$ can be calculated.

I would like to stress that such a confidence interval is an estimate for the uncertainty due to statistical fluctuations. If the real model was well approximated by the logistic regression, the confidence interval would reflect the expected statistical fluctuations. It does not cover errors due to the violation of the assumption, that the true model is well approximated, or that the model is not correct or that flaws such as changes of the dose response curve within a single experiment exist.

Furthermore, experiments were designed to measure $E_{50}$ and tested energies typically are close to $E_{50}$. This increases the uncertainty of the width $w$ when using the logistic regression. Based on empirical analysis, I will therefore assume a width $w=0.3 E_{50}$ in my analysis and only estimate $E_{50}$ by maximizing probabilities where a success was recorded and minimizing probabilities elsewhere.

\subsubsection{Syncrolation}

The new defibrillation technique that will be investigated consists of a periodic pulse sequence that has a sufficient field strength to cause synchronization followed by a single pulse of larger field strength that shall lead to the termination of the fibrillation. 


\section{Results}

This kind of pulse sequence will be named Syncrolation ${ }^{8}$ in the following. On the one hand, this pulse sequence is analogous to the one used in simulations presented in chapter 2 and the results may give hints on whether the mechanism of terminating VF with this pulse sequence has similarities with the mechanism of simultaneous unpinning of spiral waves observed in simulations. On the other hand, the periodic pulse sequence is supposed to create an ordered state and the study will show whether such a state promotes the defibrillation with a single shock.

The analogy of this method to the one presented in chapter 2 was used to roughly estimate some of the parameters of Syncrolation:

- The electric field strength of the periodic shocks shall be high enough to observed synchronization, but lower, preferably much lower, than the field strength that is needed for simultaneous activation. This is because the periodic sequence shall entrain the activity rather than replace it completely. (In simulations spirals are synchronized.) Only the last pulse shall potentially unpin spirals and lead to the termination of VF. The necessary field strength was estimated in the beginning of each experiment by recording a Synchronization Map and observing the power spectrum of the optical signal with MultiRecorder ${ }^{9}$ [1].

- In the simulations, the synchronizing pulse sequences have a higher frequency than the spiral waves. The frequency of the periodic pulse sequence will thus be chosen relative to the dominant frequency of the VF. However, it should be kept in mind that it can be misleading to assign a single frequency to the dynamics of VF as discussed in section 4.1.5.

- The time delay $\tau$ should be such that the unpinning pulse is applied in the unpinning window of pinned and synchronized spirals. We did not have means to measure this quantity in experiments ${ }^{10}$. We thus used the dominant frequency of the fibrillation as a reference.

- Monophasic rectangular pulses were used, because it is the pulse shape that was also used in simulations and the effect caused by this pulse shape is easier to understand theoretically compared to biphasic pulses.

A schematic drawing of the investigated pulse sequence depicting some of its parameters is shown in fig. 4.17. The theoretical considerations above and the results of some explorative experiments were used to get rough estimates for the parameters of Syncrolation. However, the appropriate values of many parameters were still not known precisely after those early experiments. For example, the frequency ratio that creates the strongest synchronization was not yet know ${ }^{11}$. Also, the optimal values of many parameters might change within experiments. Thus, many parameters of Syncrolation were changed from experiment to experiment or even within an experiment. The parameters that define Syncrolation and its value ranges that were used in experiments with rabbit hearts are listed below:

\footnotetext{
${ }^{8}$ The name combines Synchronization and Defibrillation.

${ }^{9}$ The more quantitative analysis of section 4.1 .5 became only available after those experiments.

${ }^{10}$ For example, there are usually no pinned spiral waves visible on the heart surface.

${ }^{11}$ The data presented in section 4.1 .5 was still being collected.
} 


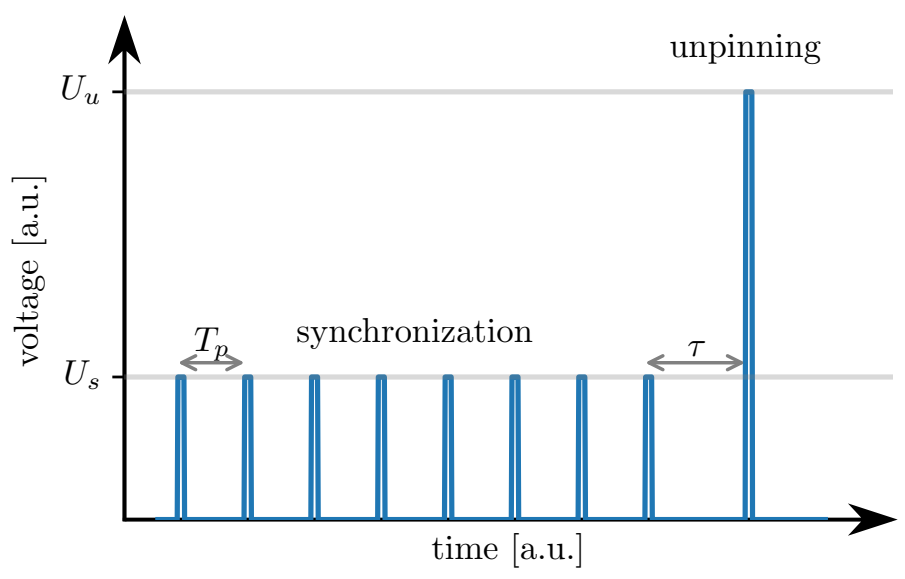

Figure 4.17: A Syncrolation pulse sequence is depicted to illustrate the main parameters: the period $T_{p}$, the delay $\tau$, and the voltages $U_{s}$ and $U_{u}$.

- voltage $U_{s}$ of synchronization pulses $\in[15,55] \mathrm{V}$

- voltage $U_{u}$ of unpinning pulse $\in[35,100] \mathrm{V}$

- pulse shape of synchronization pulses: monophasic rectangular pulses with a width of $5 \mathrm{~ms}$

- pulse shape of unpinning pulse: biphasic rectangular pulse with a width of 3 to $10 \mathrm{~ms}$, with the second phase being typically $2 \mathrm{~ms}^{12}$

- period $T_{p}$ of synchronization pulses: $T_{p}=T_{d}^{E C G} \cdot r_{T}$ with period factor $r_{T} \in$ $[0.8,1.2]$ and $T_{d}^{E C G}$ being the dominant frequency of the ECG

- time delay $\tau$ of last pulse: $\tau=T_{d}^{E C G} \cdot r_{\tau}$ with $r_{\tau} \in\{0.8,1.0\}^{13}$

- number $N_{s}$ of synchronization pulses $\in[5,30]$

Please, refer to table A.3 on page 110 for details.

In a total of 18 experiments with rabbit hearts, 662 defibrillation attempts with Syncrolation were recorded. I will refer to this dataset of defibrillation attempts as FRSyncr (Full Rabbit Syncrolation).

In experiments with pig hearts, the bath that contains the perfused heart is larger and the plate electrodes are further apart. Therefore, the voltages are different compared to those in the experiments with rabbit hearts: The synchronizing pulses have voltages from 42 to $100 \mathrm{~V}$ and the unpinning pulse has a voltage of 190 to $1000 \mathrm{~V}$. As voltages over $100 \mathrm{~V}$ are applied using a custom defibrillation device with charged capacitors for shock delivery, the pulse shape cannot be rectangular, but it is exponential. Furthermore, in many experiments with pig hearts a catheter was

\footnotetext{
${ }^{12}$ Unfortunately, the automatic logging of the duration of the second half of biphasic pulses was faulty. For some experiments only the full pulse width was stored.

${ }^{13}$ This parameter was set manually and (if at all) only corrected few times in an experiment. Thus, these values of $r_{\tau}$ should be considered to be target values.
} 


\section{Results}

inserted into the RV and the voltage was applied between the spiral electrode of the catheter and one plate electrode or between the two plate electrodes. In total 200 Syncrolation defibrillation attempts were recorded in 9 experiments with pig hearts. These recordings will be referred to as FPSyncr (Full Pig Syncrolation). Please refer to table A.4 on page 111 for details on the used parameters. Please note, that many of the experiments with pig or rabbit hearts were multi-purpose experiments, in which not only Syncrolation measurements were done.

When early experiments had promising results, this defibrillation technique was declared as an invention of three inventors with equal contributions. The inventors are Daniel Hornung, Tariq Baig-Meininghaus and myself. The invention is registered as a patent under the European publication number EP 3342455 [78].

While in both, experiments with rabbit and pig hearts, many different parameter combinations were tested, the larger set of data from experiments with rabbit hearts contains a subset of recordings with quite comparable settings. In this set of data, which will be referred to as CRSyncr (Comparable Rabbit Syncrolation), Syncrolation has 20 or 25 monophasic synchronizing pulses with a width of $5 \mathrm{~ms}$. The period factor $r_{T}$ is either 0.95 or 1.05 and the unpinning pulse is biphasic with a width of $5+2 \mathrm{~ms}$ (see table A.6 for more details).

\subsubsection{Single Shock}

Conventional defibrillation attempts (1BP) serve as a reference defibrillation technique. This method simply consists of one biphasic pulse with a rectangular (exponential) shape in experiments with rabbit (pig) hearts. It is very similar to the clinically used defibrillation method. The pulse width is either $(2.5+2.5) \mathrm{ms}$ or $(5+2) \mathrm{ms}$. The dataset consisting of the 1BP events in the experiments of the CRSyncr (FPSyncr) dataset will be referred to as $C R 1 B P(F P 1 B P)$.

\subsubsection{Five Biphasic Underdrive Pulses}

As a second reference, five periodic, biphasic pulses (5BP) are applied with a smaller frequency than the dominant frequency of the VF (underdrive). The five pulses have the same voltage and the frequency is chosen according to the detected dominant cycle length of the VF, $T_{d}^{E C G}$. The ratio $T_{p} / T_{d}^{E C G}$ is chosen to be in the interval $[1.15,1.2]$. The pulse width is either $(2.5+2.5) \mathrm{ms}$ or $(5+2) \mathrm{ms}^{14}$. The dataset consisting of the 5BP events in the experiments of the CRSyncr (FPSyncr) dataset will be referred to as $C R 5 B P(F P 5 B P)$.

${ }^{14}$ The $(\mathrm{x}+\mathrm{y})$ ms notation means that a biphasic pulse has $\mathrm{x}$ ms with the first polarity and $\mathrm{y}$ ms with the second. 

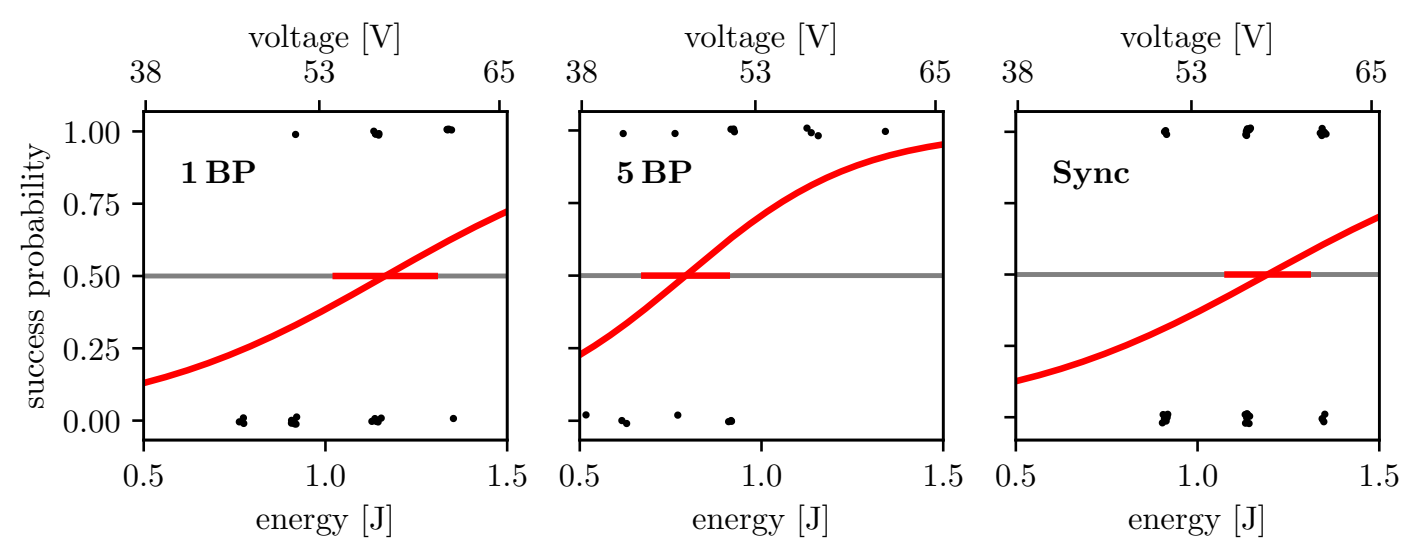

Figure 4.18: The fitted dose response curves for single shock (1BP), five underdrive pulses (5BP) and Syncrolation. The data is from the experiment with a rabbit heart on 2017-01-31. Failed defibrillation attempts are depicted by a black dot at zero, successful ones at one with an added jitter for visibility.

\subsubsection{Comparison of Characteristic Energies}

In most of the experiments, the three above described defibrillation methods were tested: 1BP, 5BP and Syncrolation. As mentioned in section 1.3, high energies of electric far field pulses used for defibrillation are associated with tissue damage and pain. An important aim of defibrillation research is thus to reduce the required energy of the electric far field shocks. Therefore, the energies of a single pulse of the methods 1BP and 5BP are compared to the energies of the unpinning pulse of Syncrolation. Of course, if Syncrolation was used as a treatment for VF in a clinical setting, also the energy of the synchronizing pulses would need to be considered. However, in this first investigation we will concentrate on the potentially more harmful energy of the unpinning pulse.

The characteristic energies $E_{50}$ will be calculated for the different defibrillation techniques by fitting a logistic model to the data from defibrillation attempts (see section 4.2.1). The characteristic energy $E_{50}^{\text {Syncr }}$ for rabbit hearts will be calculated by only considering the dataset CRSyncr with comparable Syncrolation settings. Unfortunately, the data set FPSyncr is too small and too heterogeneous to allow a further restriction to very comparable Syncrolation parameters. I will thus use the full dataset FPSyncr and the heterogeneity needs to be considered when interpreting the results. No $E_{50}$ value will be calculated for a defibrillation method if less than 5 defibrillation attempts are recorded in an experiment for that method. This is because the value would be very error prone and assumptions that are used when estimating the error are likely to be violated (see section 4.2.1).

Exemplary fits and the corresponding defibrillation outcomes are shown in fig. 4.18 for the experiment of 31 Jan 2017. Each axis displays the data corresponding to one defibrillation method. The shown defibrillation curves depict the success probability 


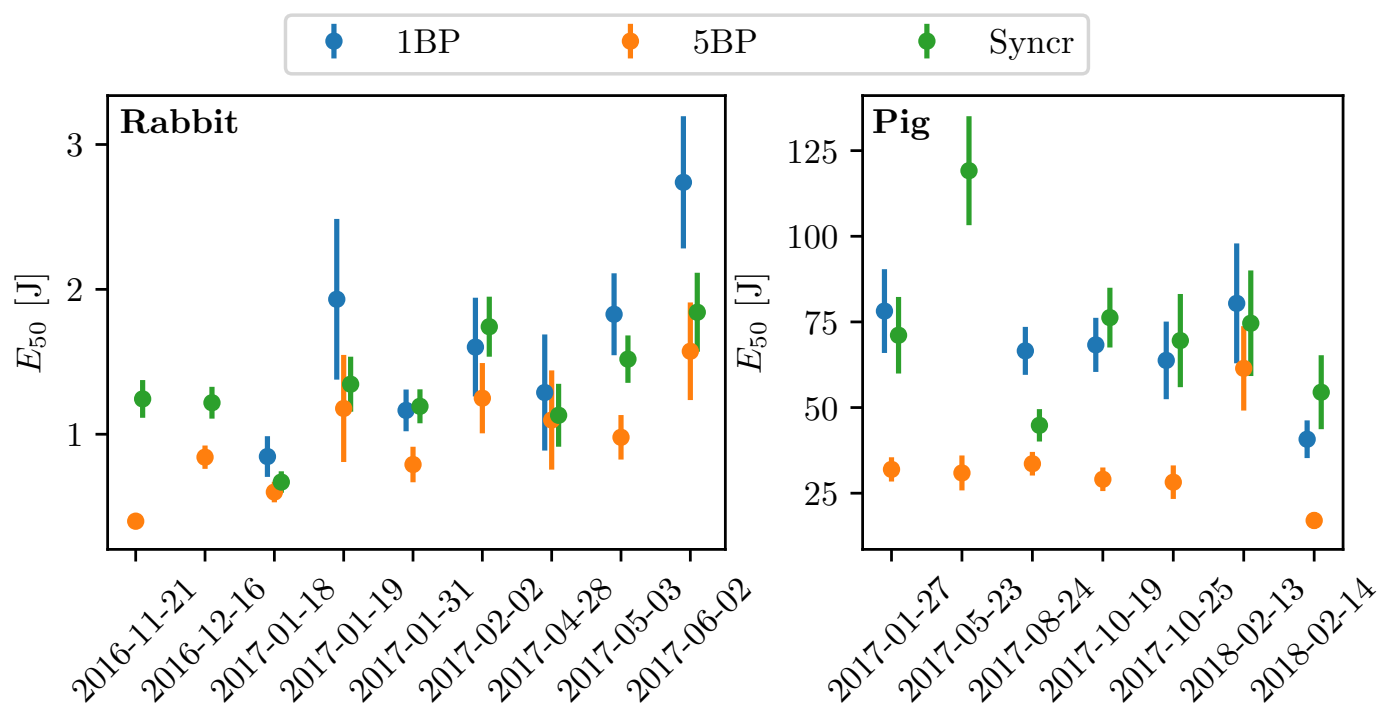

Figure 4.19: The characteristic energies $E_{50}$ are shown for the three defibrillation methods single shock, five underdrive pulses and Syncrolation for the different experiments with pig or rabbit hearts. The data points of the different methods have small horizontal offsets to increase the readability of the diagram.

depending on the energy of the pulses. As the voltage is the quantity that is controlled in the experiments, the upper axis additionally shows the corresponding voltages ${ }^{15}$. The black dots depict the outcome of single defibrillation attempts: zero stands for a failed attempt and one for a success. The error bar depicts the uncertainty of the $E_{50}$ calculated according to the Monte Carlo method described in section 4.2.1. As is intended by the experimental design, the defibrillation attempts cluster around the energies $E_{50}$. This distribution of data points leads to comparably small uncertainties of $E_{50}$. Also, in this particular experiment $5 \mathrm{BP}$ had a small $E_{50}$ value compared to $1 \mathrm{BP}$ and Syncrolation which have similar $E_{50}$ values.

An overview over the $E_{50}$ values for both pig and rabbit hearts is shown in fig. 4.19 (see also table A.5). In the experiments of 21 Nov 2016 and 16 Dez 2016 no defibrillation attempts with a single shock were recorded as the advantage of comparing 1BP and Syncrolation was only recognized after those experiments. Also, the experiment of 15 Feb 2018 is excluded as too few Syncrolation defibrillation attempts are recorded to allow the calculation of an $E_{50}$ value.

While the energies of $1 \mathrm{BP}$ and Syncrolation are often comparable, the energies of 5BP are usually smaller. In the experiment of $24 \mathrm{Aug} 2017, E_{50}^{\text {Syncr }}$ is comparably small. However, in that particular experiment the pulse shape of the unpinning pulse was accidentally set to a symmetric shape, i.e. the second part of the biphasic pulses starts at the same voltage as the first part. In none of the other recordings of any

${ }^{15}$ It was so far not feasible to determine the resistance which is not recorded directly. Therefore, a typical resistance of $20 \mathrm{Ohm}$ is assumed for the conversion. 
method a symmetric pulse shape was used. Thus, the small $E_{50}$ value of Syncrolation in this particular experiment might be due to the changed pulse shape.

If there was no difference in defibrillation probability for an equal energy of $1 \mathrm{BP}$ and the unpinning pulse of Syncrolation (i.e. $E_{50}^{1 B P} \approx E_{50}^{S y n c r}$ ), then the periodic pulse sequence of Syncrolation would be meaningless as it would not provide any benefit. Let us thus test the hypothesis $E_{50}^{1 B P}>E_{50}^{\text {Syncr }}$ (Hypo1) using the data from rabbit experiments and the data from pig experiments, separately.

\section{Procedure of the Hypothesis Test}

The null hypothesis to the hypothesis Hypo1 is that the $E_{50}$ values of the two defibrillation techniques are the same. The test statistic $Z=E_{50}^{1 B P}-E_{50}^{\text {Syncr }}$ is zero when the null hypothesis is true. Similar to the case when we were estimating the uncertainty of $E_{50}$ values, we face again the problem that we have no analytic expression for $Z$. I will therefore proceed as before and generate an estimate of the distribution of the test statistic from simulated experiments (see section 4.2.1).

The logistic model that needs to be evaluated has two parameters: the characteristic energy $E_{50}$ and the width of the transition $w$. I will here additionally assume that not only the characteristic energies, but also the width $w$ is the same under the null hypothesis. Thus we can estimate a common model for the combined dataset of both defibrillation methods under the null hypothesis. This is done as before using a logistic regression.

Let us denote the number of recorded defibrillations of the one method by $n$ and the number of attempts of the other method by $m$. Using the estimate of the common dose response curve under the null hypothesis, I simulate experiments with $m+n$ observations, calculate one $E_{50}$ value from $n$ observations and one from the other $m$ observations. This provides one realization of $Z$ for each simulated experiment and thereby produces the distribution of the test statistic. In the following analyses, 5000 experiments are simulated in order to get an estimate of the distribution of the test statistic. The observed value of the test statistic $Z$ is then compared to the distribution and thus provides a p-value for each experiment. The p-values of the single experiments are combined to one p-value to test the common hypothesis using Stouffer's method (see [71] for a review on this and other methods for combining p-values). Experiments where no comparison of characteristic energies is possible, e.g. because one of the $E_{50}$ values could not be determined, are excluded when performing Stouffer's method.

\section{Result of the Hypothesis Test}

The p-values of the single experiments and the combined p-value are shown in table 4.1. The combined p-value is 0.04 and 0.6 for the data from rabbit and pig hearts, respectively. 


\begin{tabular}{|c|c|c|c|}
\hline $\begin{array}{l}\text { Rabbit } \\
\text { date }\end{array}$ & $\mathrm{p}$-value & $\begin{array}{l}\text { Pig } \\
\text { date }\end{array}$ & $\mathrm{p}$-value \\
\hline 2017-01-18 & 0.15 & 2017-01-27 & 0.33 \\
\hline 2017-01-19 & 0.12 & 2017-05-23 & 0.91 \\
\hline 2017-01-31 & 0.56 & 2017-08-24 & 0.004 \\
\hline $2017-02-02$ & 0.61 & 2017-10-19 & 0.75 \\
\hline $2017-04-28$ & 0.37 & 2017-10-25 & 0.64 \\
\hline 2017-05-03 & 0.20 & 2018-02-13 & 0.40 \\
\hline 2017-06-02 & 0.04 & 2018-02-14 & 0.93 \\
\hline combined & 0.04 & combined & 0.6 \\
\hline
\end{tabular}

Table 4.1: The p-values of the test whether $E_{50}^{1 B P}>E_{50}^{\text {Syncr }}$ are shown for each experiment in CRSyncr. The combined p-value for the whole experiment series is shown in the bottom row and was calculated with Stouffer's method.

The significance level is chosen to be $\alpha=0.05$. This is one of two hypotheses that will be tested here. Thus the p-values of single hypotheses will be tested against a corrected significance level $\alpha^{\prime}=\alpha / m$ with $m=2$ being the number of hypotheses according to the Bonferroni correction.

Both values are larger than the significance level, i.e. the null hypothesis cannot be rejected in any of the two cases. This means, that the data from these 14 experiments with rabbit and pig hearts does not allow to exclude the possibility that the energy of the unpinning shock in Syncrolation required for $50 \%$ success rate is the same as the one of single shock defibrillation. In other words, the possibility that the preceding periodic pulses do not provide a beneficial effect for defibrillation cannot be excluded.

Please, note that the date of 24 Aug 2017 was included in the analysis even though one could argue, that the data should be excluded due to the different pulse shape used for Syncrolation (see above). However, the exclusion would only increase the common p-value and thus not change the result.

However, the above analysis is for recordings of Syncrolation defibrillation attempts with differing parameters and some parameters might have been chosen poorly. According to the design of Syncrolation the initial series of pulses shall synchronize the tissue. This has not yet been checked. We know from section 4.1.5 that such synchronization depends on the ratio of the pacing frequency and the frequency of the activity of the heart. Therefore, I will test the influence of the parameter $r_{T}$, defined as the ratio of the pacing period and the dominant cycle length of the fibrillation, on the defibrillation success in the next section. 


\begin{tabular}{|c|c|c|c|c|c|}
\hline Rabbit & $r_{T}=0.95$ & $r_{T}=1.05$ & Pig & $r_{T}=0.95$ & $r_{T}=1.05$ \\
\hline & 58 & 65 & success & 31 & 5 \\
\hline failure & 75 & 73 & failure & 37 & 15 \\
\hline
\end{tabular}

Table 4.2: The contingency table for Hypo2. The number of successful and failed defibrillation grouped by the leap factor. The shown data is from the CRSyncr dataset and the FPSyncr dataset restricted to $r_{T} \in\{0.95,1.05\}$.

\subsubsection{Influence of the Period Factor}

In the simulations where multiple spirals were simultaneously unpinned, the pacing period was smaller than the period of the spirals. This leads to synchronization of the spirals. In analogy to this, a pacing period $T_{p}$ that is faster than the dynamics of the VF should lead to a larger synchronization. Furthermore, I demonstrated in section 4.1.5 that a periodic pulse sequence with a pacing frequency about 1.1 times faster than the dominant frequency of the VF causes the strongest synchronization. And if a high synchronization promotes the defibrillation with a single pulse, then the frequency ratio should influence defibrillation probability.

In a Syncrolation defibrillation attempt, the ECG just before the onset of the pulses is analyzed and the dominant cycle length is estimated using a sine fitting algorithm (see section 3.4). The pacing frequency $1 / T_{p}$ is then set according to the detected dominant cycle length $T_{d}^{E C G}$ and the period factor $r_{T}: T_{p}=r_{T} \cdot T_{d}^{E C G}$.

Two different period factors $r_{T}$ are contained in the dataset CRSyncr with comparable Syncrolation settings $(0.95$ and 1.05). Let us therefore test the hypothesis that Syncrolation with a period factor of 0.95 has a different success rate than Syncrolation with a period factor of 1.05 (Hypo2).

The experimental protocol enforces that always pairs of defibrillation attempts are recorded in which Syncrolation is applied with the same voltage of the unpinning pulse $U_{u}$ and both values of the period factor $r_{T}$. This assures on the one hand that both $r_{T}$ values are uniformly used throughout the experiment and more importantly that the used energies of the unpinning pulse are (when possible) the same in both groups. Indeed, the mean pulse amplitude is 58.2 and $58.9 \mathrm{~V}$ in the two groups. Thus, it is unlikely that a difference in success rate should be attributed to the amplitude of the unpinning shock.

The contingency tables for both rabbit and pig data is shown in table 4.2. The success rates appear to be very similar for both groups and indeed a chi-squared test provides a p-value of 0.56 for the rabbit data and 0.1 for the pig data. This means that the null hypothesis cannot be rejected, i.e. there is no evidence that pulse sequences with periods smaller than the dominant cycle length $T_{d}^{E C G}$ have a different success rate than those with larger periods. Additionally to the result that the energies $E_{50}$ of 1BP and Syncrolation are similar, this also suggests that the initial periodic pulse sequence does not provide a substantial benefit to defibrillation. 

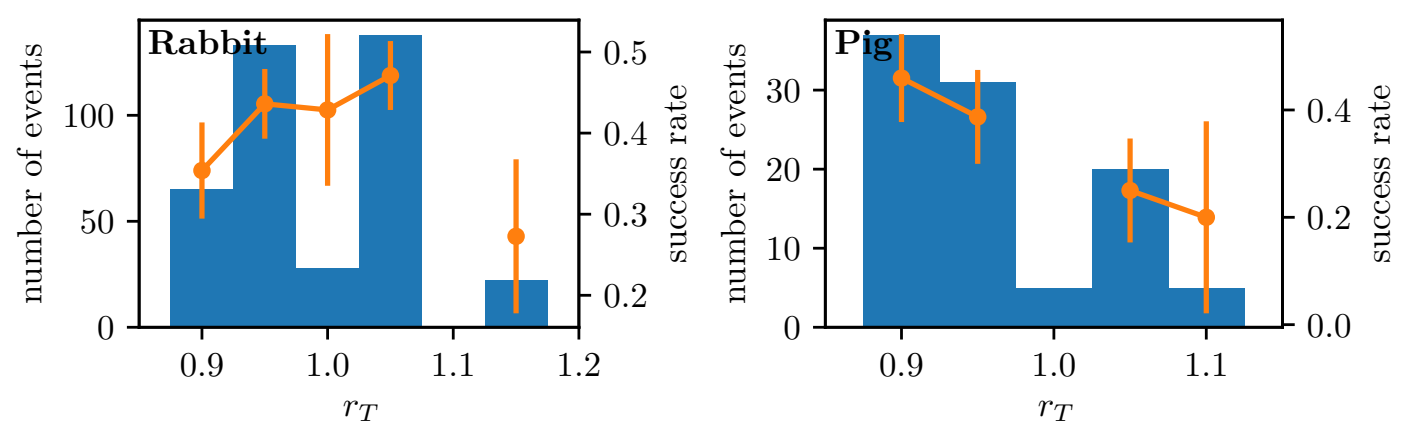

Figure 4.20: The success rates of Syncrolation with respect to the period factor $r_{T}$ is shown for data recorded from rabbit (left) and pig (right) hearts. The blue bars indicate the number of defibrillation attempts in each bin. The success rates and the corresponding standard error are depicted in orange. Success rates are not shown if their uncertainty cannot be estimated. In the rabbit data, the success rates of $r_{T}=0.9$ and $r_{T}=1.15$ are smaller than the others. In the pig data, the success rates are larger for smaller values of $r_{T}$.

So far I have only considered Syncrolation defibrillation attempts with a period factor $r_{T} \in\{0.95,1.05\}$, as these are the only values in the data set CRSyncr. Figure 4.20 however shows the success rates of Syncrolation in the datasets FRSyncr and FPSyncr grouped by the period factor $r_{T}$. While the diagram with the pig data shows an increase in success rate towards smaller values of $r_{T}$, the diagram with the rabbit data indicates that the largest success rates are found around a period factor $r_{T}=1$ : The success rates for small values $\left(r_{T} \approx 0.9\right)$ and large values $\left(r_{T} \approx 1.15\right)$ are smaller than the others.

On the one hand the increased success rate for small values of $r_{T}$ in the data from experiments with pig hearts stands in contrast to the result of the test of Hypo2, where no significant difference in success rate was found. On the other hand, the data from experiments with rabbit hearts is an indication that no significant difference in success rate was found when testing Hypo2 because the success rate seems to have a maximum around $r_{T}=1$ such that the difference of success rates for $r_{T}=0.95$ and $r_{T}=1.05$ is minimal.

In the following, I will test the statistical significance of these trends. However, the experiments were not designed to measure these trends. Additionally, the parameters used for Syncrolation in these datasets (FRSyncr and FPSyncr) vary strongly which bears the risk that the trends are caused by some other factor that was not accounted for. Due to this and due to the multiple testing at hand, the reader should not interpret the following p-values as statistical proof, but solely as a motivation to conduct further experiments to verify these findings.

Let us compare the two groups $r_{T}<1$ and $r_{T} \geq 1$ in the data from experiments with pig hearts. The mean $U_{u}$ value of the data for $r_{T}<1$ is $\approx 860 \mathrm{~V}$, while the one for $r_{T} \geq 1$ is $\approx 910 \mathrm{~V}$. As a high voltage should always lead to a higher success rate, this 
difference can counteract the difference in success rate seen in fig. 4.20 but it cannot explain it. A chi-squared test for difference of the two groups $r_{T}<1$ and $r_{T} \geq 1$ results in a p-value of 0.03 .

When we compare the success rates of the data from experiments with rabbit hearts with $0.95 \leq r_{T} \leq 1.05$ to the remaining data, we find that the mean voltage of the centered data with $0.95 \leq r_{T} \leq 1.05$ is $59 \mathrm{~V}$ and the mean voltage of the remaining data is $66 \mathrm{~V}$. Again, this difference in the mean voltage cannot cause the different success rates. The p-value of a corresponding chi-squared test is 0.05 .

These findings might be a hint that the period factor $r_{T}$ does influence the success rate of Syncrolation. This is in contrast to the above described result of the test of Hypo2, where no significant difference between the success rates for $r_{T}=0.95$ and $r_{T}=1.05$ was found. The contradicting result, may be due to the unexpected location of the maximum success rate in the data from rabbit hearts and because the difference of the success rate is smaller for the originally tested values (0.95 and 1.05) than for $r_{T}=0.9$ and $r_{T}=1.1$ in the data from pig hearts (see fig. 4.20). One additional finding supports this view, that I will describe in the following.

The synchronization of cardiac activity by periodic electric far field pulses was studied in section 4.1.5. It was shown that the ratio of the pacing frequency and the dominant frequency of the dynamics strongly influences the strength of the synchronization. However, there is an important difference to the analysis of the section: The measure that was considered in section 4.1.5 is the mean dominant frequency of the optical signal $\bar{f}_{d}^{o p t}$ while I here consider the frequency calculated from the ECG, $1 / T_{d}^{E C G}$. This difference would not matter if both frequency measures were identical or very similar. However, comparing the two measures (see fig. A.1) shows that there is a considerable difference in the data from experiments with rabbit hearts: Above a frequency of $\approx 8 \mathrm{~Hz}$, the optical frequency $\bar{f}_{d}^{o p t}$ is often smaller than the electrical dominant frequency $1 / T_{d}^{E C G}$ and this deviation increases for higher frequencies. In contrast, the data recorded in experiments with pig hearts shows that the two measures are very similar and the data points in fig. A.2 lie near to the identity.

The difference of the ECG measures and the optical measure might be caused by regions of heart tissue that cannot be activated with high frequencies. If those regions were for example activated every second time, then the dominant frequency would be half of the value in the remaining tissue and the mean $\overline{f_{d}}$ opt would be decreased. The ECG signal that also depends on the electric currents in the tissue bulk might be less affected by the slow regions. This would lead to the above described difference of the frequency measures.

Thus, the ratio $f_{p} /{\overline{f_{d}}}^{o p t}$ used in fig. 4.8 can be greater than $f_{p} \cdot T_{d}^{E C G}=T_{d}^{E C G} / T_{p}=$ $1 / r_{T}$ for data recorded from rabbit hearts. In fig. 4.8 we see the highest synchronization for $f_{p} /{\overline{f_{d}}}^{o p t} \approx 1.1$. This means that the maximum synchronization can be expected for $1 / r_{T}<1$.1. Suppose the maximum synchronization is shifted such that the success rate decreased similarly towards $r_{T}=0.95$ and $r_{T}=1.05$, then the choice to compare those values in Hypo2 would be especially unfortunate. 


\section{Results}

This might be a reason why no difference in success rate was found in the dataset CRSyncr with comparable Syncrolation settings and could explain that the maximum success rate is found for $r_{T} \approx 1$ for data in fig. 4.20 .

Summarizing, I did not find statistical evidence that the period factor $r_{T}$ influences the success rate of Syncrolation when comparing the defibrillation attempts with $r_{T}=0.95$ to the ones with $r_{T}=1.05$. This supports the interpretation that the initial synchronizing pulse sequence of Syncrolation does not promote the defibrillation. However, a difference of the here used frequency measure and the one used in the analysis of section 4.1.5 might have caused an unexpected shift of the high success rate peak from $r_{T} \approx 0.9$ towards $r_{T} \approx 1$ such that unfavorable values might have been chosen for the comparison. Figure 4.20 in contrast does indicate a dependency of the success rate on the period factor $r_{T}$. The corresponding datasets are however very heterogeneous which is the main reason why this should be a seen as a motivation to test this finding in additional experiments rather than interpreting it as statistical proof.

\subsubsection{Influence of the Delay}

Another Syncrolation parameter that was varied in the experiments is the time delay of the last pulse $\tau$. In the simulations presented in chapter 2, the time delay $\tau$ was a crucial parameter for the unpinning of spiral waves. But the relevant quanitity, the location of the unpinning window, cannot be measured in experiments. The hypothesis to be tested in this section is that the defibrillation success rate of Syncrolation depends on $\tau$. It is however unclear whether this is a dependence on the absolute value or rather on the delay $\tau$ relative to some characteristic time of the tissue. This characteristic time could for example be the mean duration of excitation during VF. Due to technical restrictions $\tau$ was therefore adjusted in some experiments relative to the dominant cycle length of the VF calculated from the ECG.

This adjustment leads to the comparable broad distribution of values in the data from experiments with rabbit hearts (see fig. 4.21). However, this was rarely done in
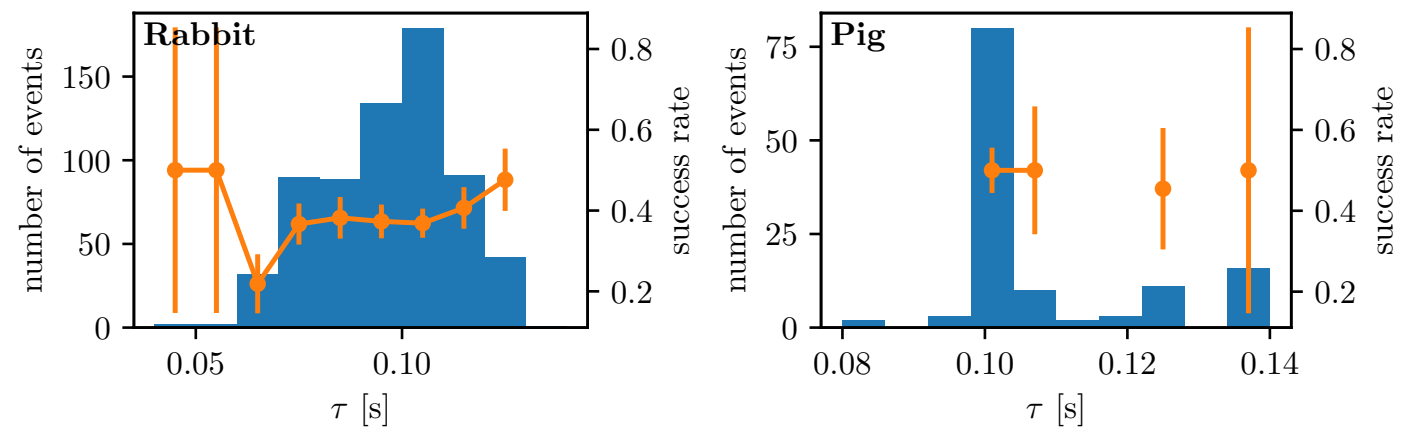

Figure 4.21: The distribution of delay times of the last pulse relative to the corresponding dominant frequency, Rabbit data on the left, pig on the right. See also the caption of fig. 4.20 . 
pig experiments such that basically only $\tau \approx 100 \mathrm{~ms}$ was used. Therefore, the data recorded from pig hearts does not allow to draw conclusions about favorable absolute values of $\tau$. The data from experiments with rabbit hearts shows that $\tau$ does not have a considerable influence on the success rate of Syncrolation (in the range of values where most measurements were done). The very small and very large $\tau$ values in the data recorded from rabbit hearts might indicate a deviation from this uniform success probability, but as only few defibrillation attempts contribute to these values no definite statement is possible.

In the experiments where $\tau$ was adjusted to the electric dynamics of the heart, it was aimed at using $\tau / T_{d}^{E C G} \in\{0.8,1\}$. This is also reflected in fig. 4.22 since the data points are distributed around these ratios. Figure 4.22 shows the distribution of delay times of the last pulse $\tau$ relative to the corresponding dominant electric cycle length $T_{d}^{E C G}$. As in the diagrams showing the absolute values, fig. 4.22 does not show a clear dependency of the success rate on the ratio $\tau / T_{d}^{E C G}$; neither in the data from experiments with rabbit hearts nor in the data from experiments with pig hearts.
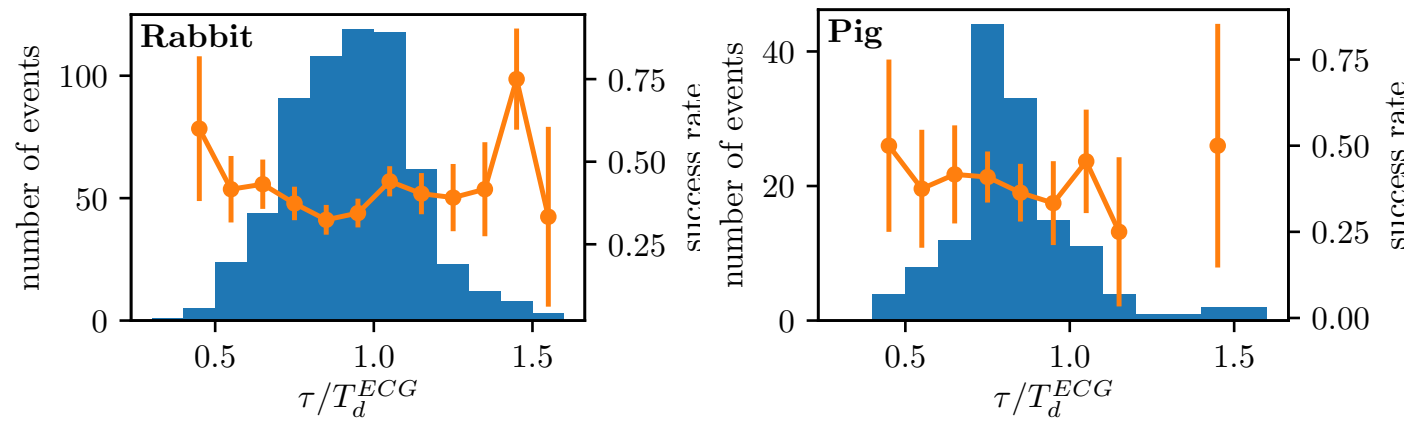

Figure 4.22: The distribution of delay times of the last pulse relative to the corresponding dominant frequency. Data from experiments with rabbit hearts is shown on the left, from experiments with pig hearts on the right. See also the caption of fig. 4.20 .

If the timing of the unpinning pulse can be completely arbitrary and is not affecting the success rate of Syncrolation, it seems unlikely that the synchronizing pulses play a role in the termination process or that the unpinning pulse does actually unpin spirals as such unpinning should be phase and thus also time dependent. For the same reason the timing of the unpinning pulse should only play a role if the synchronizing pulses of Syncrolation actually synchronized the tissue. Therefore I restricted the above data in a new analysis to those defibrillation attempts where the synchronizing pulses of Syncrolation caused a mean synchronized area fraction of at least 0.7 during the last $1 / 8$ of the periodic pacing sequence (see section 4.1.5). Additionally, when large fractions of the tissue are entrained by the periodic pulses, it seems unreasonable to use the dynamics before the entrainment as a reference for the delay of the unpinning pulse $\tau$. Instead of the ratio $\tau / T_{d}^{E C G}$ I will thus in the following analyze the ratio influence of $\tau / T_{p}$ on the success rate of Syncrolation. 

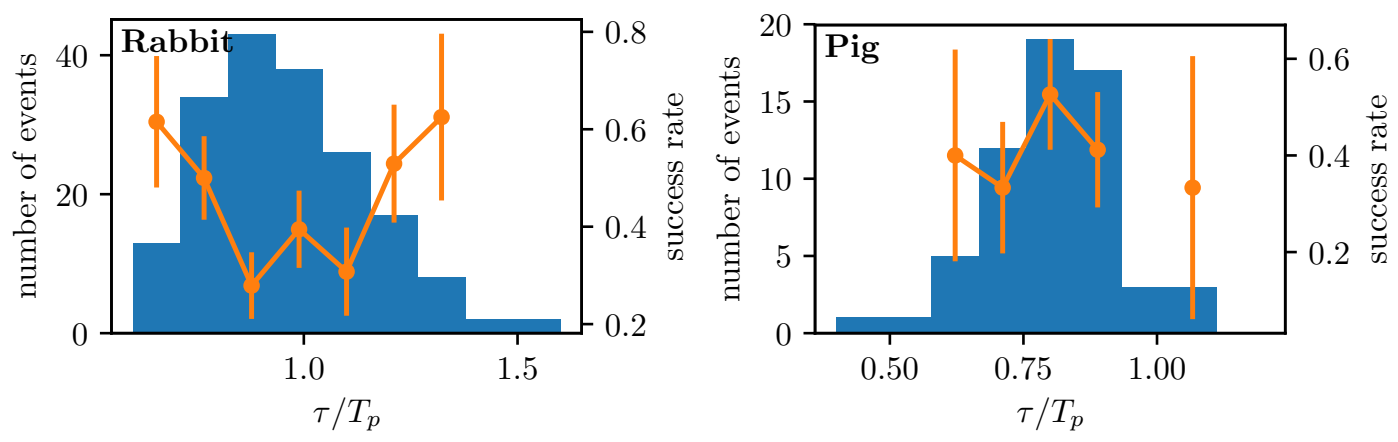

Figure 4.23: The number of defibrillation attempts and the success rates are shown relative to ratio of the delay $\tau$ and the pacing frequency $T_{p}$ for data recorded in experiments with rabbit (left) and pig hearts (right). See also the caption of fig. 4.20. In the data from experiments with rabbit hearts, most defibrillation attempts were performed with a ratio $\tau / T_{p} \approx 1$, while the success rate is the smallest around this value of the ratio. Defibrillation attempts recorded from pig hearts have mostly other values of the ratio $\tau / T_{p}$ and do not show a clear dependency of the success rate on this ratio.

Figure 4.23 displays the dependency of the success rate of Syncrolation on the ratio $\tau / T_{p}$. In the data from rabbit experiments, a large drop in success rate for a ratio $\tau / T_{p} \approx 1$ can be seen. The ratio $\tau / T_{p}$ in the data from experiments with pig hearts lies mostly in a different range compared to data recorded from rabbit hearts. The data from experiments with pig hearts thus neither supports nor contradicts the finding based on the data from rabbit hearts.

Interestingly, the low success rates in the data of experiments with rabbit hearts are around a ratio of 1 . This means that the unpinning pulse has the smallest probability to terminate the VF when it is applied after the same period as the periodic pulses before. On the one hand this fits into the picture of unpinning spirals. In chapter 2 , the unpinning pulse was not applied after a full period. On the other hand, if an unpinning pulse is applied after $\tau \approx T_{p}$, then the pulse will mostly excite the same tissue as the periodic pulses before. Thus a deviation from this periodic activation seems to be advantageous for terminating VF.

\subsubsection{Synchronization as a Predictor for Termination}

When designing Syncrolation, the periodic pulse sequence was intended to synchronize the electric dynamics of the heart. Thus it is a natural expectation that Syncrolation has a large success rate when the periodic pulses actually synchronize the tissue. Figure 4.24 shows the success rate of Syncrolation binned according to the mean synchronized area fraction in the last $1 / 8$ of the periodic pacing sequence for the three data sets FRSyncr, CRSyncr and FPSyncr. In contrast to the initial assumption, 

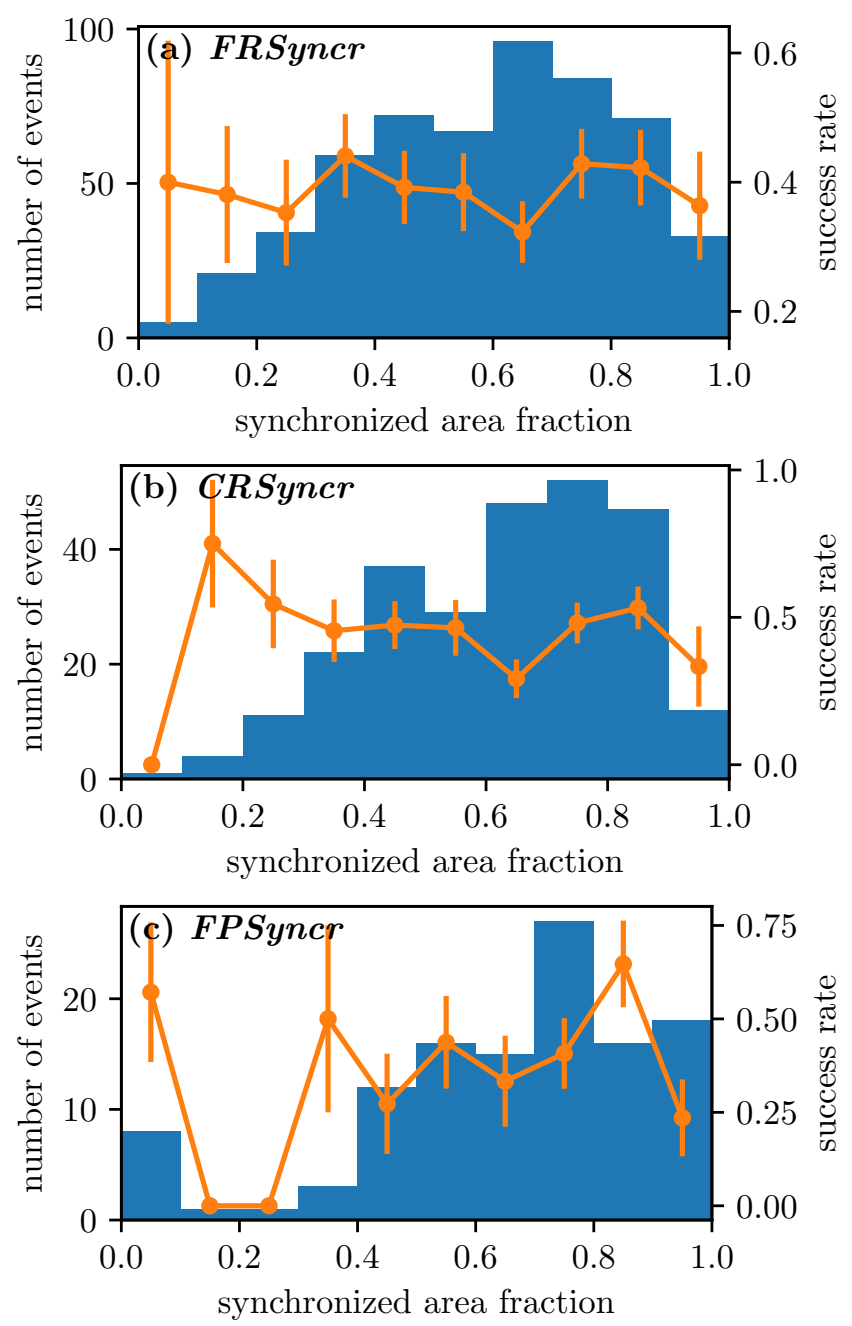

Figure 4.24: The number of defibrillation attempts and the respective success rate is shown relative to the synchronized area fraction during the last eighth of the periodic pulse sequence of Syncrolation. The three diagrams correspond to the three datasets FRSyncr, CRSyncr and FPSyncr. See also the caption of fig. 4.20. Clearly, a large number of defibrillation attempts does not have a high synchronized area fraction even though the periodic sequence is supposed to create a high synchronization. Furthermore, no dependency of the success rate on the synchronized area fraction is visible. 


\section{Results}

there is no clear increase of the success rate towards larger values of the synchronized area fraction in either of the three datasets.

Results of section 4.2.7 indicated that a $\tau$ value that is near 0.8 or 1.2 leads to high success rates when the synchronized area fraction is above 0.7 . However, most of the recordings were done for $\tau \approx 1$ where a low success rate was found. It is thus possible that no dependence of the synchronized area fraction is seen in fig. 4.24 because the effect is diminished by the frequent usage of unfavorable $\tau$ values are used.

\subsubsection{Section Summary}

Most results of the experiments with Syncrolation defibrillation attempts are surprising and different from what was originally expected:

1. The characteristic energy of the unpinning shock required for $50 \%$ success rate could not be shown to be smaller than the one of a single shock.

2. No difference in the success rate was found for the two different frequency ratios $r_{T}$ in the dataset with comparable settings of Syncrolation CRSyncr.

3. The synchronization measured using the synchronized area fraction did not prove to be a good predictor for the defibrillation success.

First, if the characteristic energy of Syncrolation is smaller than the one of a single shock defibrillation, then this difference is so small that the test result is not significant. This shows that Syncrolation is, in the tested configuration, not beneficial compared to single shock defibrillation and it is very unlikely that the additional, synchronizing pulse series is meaningful.

Second, the obvious conclusion is that the mechanism that leads to the termination in those defibrillation attempts differs substantially from the simultaneous unpinning of multiple spirals.

However, the possibility that Syncrolation can be beneficial in some different configuration and that unpinning does play a role cannot be ruled out. We saw some flaws in the experiment design that might have caused the above results, especially by reducing the effect size.

1. The synchronization pulses are supposed to create sufficient synchronization but often did not.

2. The data suggests that a comparison of success rates at e.g. a period ratio $r_{T}=1$ and $r_{T}=1.2$ would be more meaningful due to a shift of the maximum success rate to $r_{T} \approx 1$.

3. When high synchronization was reached, time delays $\tau$ of the unpinning pulse were often chosen unfortunately. 
Even though additional experiments are necessary to provide statistical evidence for these findings, they provide a different plausible explanation for the results of the experiments. In contrast to the time when the previous experiments were designed, I do now know for what parameters of periodic pulse sequences a maximum synchronization can be expected. I thus suggest to conduct experiments where the characteristic energies $E_{50}$ of Syncrolation and a single shock are compared. In those experiments, the parameters of Syncrolation should assure that a high synchronization is usually achieved and the time delay $\tau$ is either much smaller or greater than 1 (see fig. 4.23). If no significant difference in the characteristic energies is found in such experiments, the possibility that a synchronizing pulse series promotes a single shock defibrillation can be ruled out.

Although these results neither proved the tested kind of Syncrolation to be an advantageous defibrillation technique nor they indicated a relevance of the mechanism that was investigated in simulations, i.e. the unpinning of multiple spiral waves, I do believe that this data provides interesting insights. Especially the finding of section 4.2.7 that in synchronized systems the delay of the last pulse $\tau$ does have a strong influence on the termination rate (although this finding is not yet statistically sound) opens interesting questions: Why is a final stimulation especially disadvantageous for defibrillation if it is applied after the same pacing period? However, this question is difficult to answer without a better understanding of the activity in the heart during these stimulation sequences and without a better mechanistic understanding defibrillation. In the following section, I will try to shed some light onto these aspects.

\subsection{Mechanisms of Defibrillation}

The results from experiments that I considered so far were aggregated quantities like characteristic energies. However, the optical mapping allows to observe the evolution of activation waves on the heart surface directly. This provides a better understanding of what is happening during the control that was discussed so far. In the following, I will present two case studies that illustrate different mechanisms of defibrillation.

In the first, a periodic sequence of pulses creates a very homogeneous activation and leads to the termination of VF. The second case study, presents a Syncrolation defibrillation attempt that fails even though a high synchronization was reached during the periodic pulsing and the unpinning pulse caused the activation of a large fraction of the tissue.

Beforehand, I would like to note that such analysis faces a few inherent difficulties: The optical mapping data only provides information about the activity on the surface of the heart. This incomplete view as well as the stochastic nature of VF need to be kept in mind when interpreting case studies as the following ones. 


\section{Results}

\subsubsection{Simultaneous Activation}

If periodic pulses have a strong synchronizing effect, then those pulse sequences can lead to termination of VF as fig. 4.3 illustrates. In fig. 4.3, the data corresponds to pulse sequences with various voltages. Still, defibrillation can occur at comparably low voltages. One such example shall be discussed here in detail.

In the experiment with a pig heart on 15 Feb 2018, periodic pulse sequences with small voltages were applied with a period factor $r_{T}=1$ in order to terminate VF. Several pulse sequences with a voltage of $85 \mathrm{~V}$ were recorded that lead to the termination of VF. The voltage of $85 \mathrm{~V}$ is much lower than the voltage of roughly $600 \mathrm{~V}$ that was necessary to obtain $50 \%$ success rate for single shock termination a short time later ${ }^{16}$. In the following, I will present the dynamics of one such defibrillation attempt (recorded at 21:04:01) with a periodic pulse sequence with $85 \mathrm{~V}$ and provide an explanation, how defibrillation was possible at this very low voltage. A video of the full recording and corresponding DFMs are available online [77].

Figure 4.25 shows optical mapping data recorded during the respective pulse sequence. The images in fig. 4.25 display the optical mapping signal at various time points with yellow color indicating activation. The first frame shows the complex pattern of activation during the VF before the first pulse. The following 10 frames show the signal just before the application of every second pulse of a total of 20 pulses that were applied. The last frame, however, shows the state, just after the application of the last pulse. Below the images, a graph displays the Pseudo-ECG, i.e. the mean value of the optical mapping data for each frame and a time series that indicates the times when pulses are applied (orange curve). The times of the frames shown in the images are indicated by gray lines with labels.

It can be seen how the periodic pulses successively remove the complex activation patterns of the VF. After six pulses, no complex patterns remain but only small areas of activated tissue. Due to the small voltage, the pulses cannot activate regions that were activated just before the pulse was applied. The activation that prevents full activation of the tissue by the application of the shocks is not stationary but moves as can be seen in the images with $t_{4}$ to $t_{8}$. After 16 pulses $\left(t_{8}\right)$, no activity on the heart surface can be seen before the pulses are applied (see $t_{9}$ and $t_{10}$ ) and the following pulses can thus excite the whole tissue. The last frame illustrates this for the last pulse as it activates the whole tissue. This sequence terminates the VF, which can be seen from the flat line of the Pseudo-ECG after the last pulse, showing that no activity remains.

An interesting mechanism can be identified in the images of fig. 4.25: The voltage of the pulses is not large enough to activate large areas of tissue at once (as it is required for single shock termination). However, after a large number of pulses the tissue is synchronized to an extend that the low-energy pulses activate the tissue

${ }^{16}$ Shortly afterwards, single shock defibrillation attempts were twice unsuccessful at $570 \mathrm{~V}$, once successful and once unsuccessful at $620 \mathrm{~V}$ and once successful at $680 \mathrm{~V}$. 


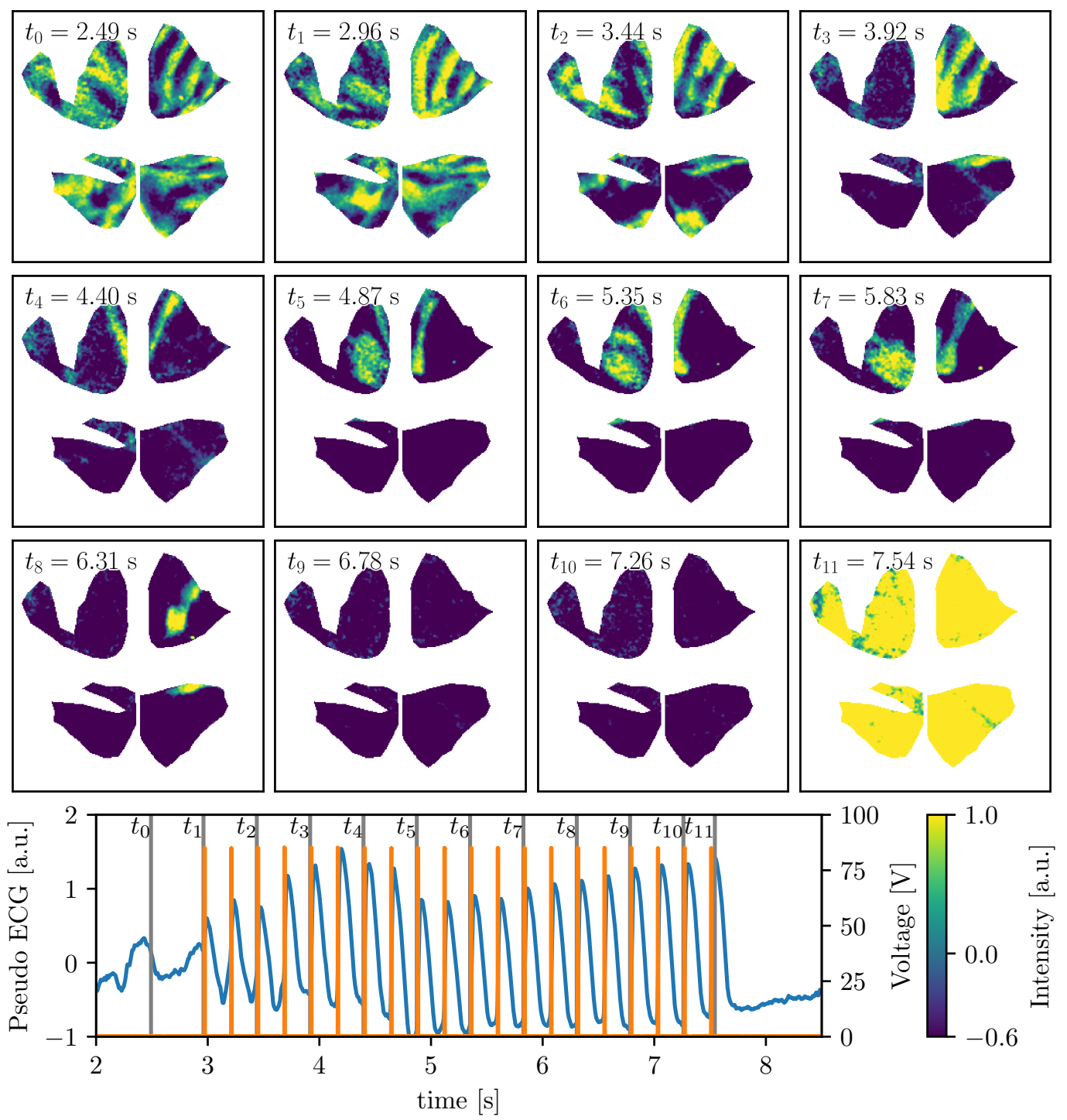

Figure 4.25: The termination of VF in a pig heart by the application of a long sequence of low energy pulses is shown. The images display the optical mapping signal at various time points with yellow color indicating activation. The first frame shows the complex pattern of activation during the VF before the first pulse. The following 10 frames show the signal just before the application of every second pulse of a total of 20 pulses with $85 \mathrm{~V}$. The last frame however shows the state just after the application of the last pulse. Below the images, a graph shows the Pseudo-ECG in blue and indicates the times when pulses are applied in orange. The times of the depicted frames are indicated by gray lines with labels. It can be seen how the periodic pulses successively remove the complex activation patterns of the VF and then successively capture the whole tissue such that the whole tissue is activated by the last pulse. 


\section{Results}

simultaneously $^{17}$, which leads to the termination of the VF. It is likely that the pulse sequence would not have terminated the VF, if only $15\left(t_{8}\right)$ or fewer pulses would have been applied because the activity that remained after the 15. pulse would probably have propagated through the tissue and continued the VF by breaking up into many waves.

Figure 4.9 shows the synchronization of cardiac activity depending on the voltage of the pulses and on the ratio $f_{p} / \overline{f_{d}}$ based on the Synchronization Map data of the experiment performed on 15 Feb 2018. In this experiment, also the above described pulse sequence was recorded. Analogous to the results reported in section 4.1.5, the highest synchronization at low voltages is found near a ratio of 1.1. However, the overall synchronization is larger than for example in the experiment of 23 May 2018 (comparable settings and experiment conduction; see fig. 4.10) or compared to the average in fig. 4.8. The animal model is different in those two experiments (chronic heart failure model versus chronic myocardial infarction), which might be the reason for the different strength of synchronization. This indicates that a defibrillation as it was shown in fig. 4.25 might not be possible in all pig models or even only in hearts of single animals.

The here presented dynamics during a periodic pulse sequence demonstrate that periodic pulses can lead to defibrillation even when small voltages are used. Apparently a successive increase of the area activated by the pulses leads to simultaneous activation of the whole tissue such that no activity can persist and the VF is terminated. It is thus necessary to know how the tissue is synchronized efficiently (see section 4.1.5) in order to make use of this mechanism and choose optimal parameters for periodic pulse sequences that shall terminate VF. However, this example also shows that a quite large number of pulses might be necessary until simultaneous activation occurs. This stands in contrast to the comparatively fast increase of the synchronized area fraction presented in section 4.1.6 that is followed by a plateau of maximum synchronization.

\subsubsection{Homogeneous activation}

In the Syncrolation defibrillation attempts recorded from rabbit hearts, large simultaneous activations, like the ones in the previous section, are rarely seen. Most of the time the synchronizing pulses can only activate some areas and large traveling waves propagate over the tissue after the activation of the pulse. The state that is reached due to the periodic pulses is thus a fundamentally different one compared to the one describe in the previous section. Figure 4.26 shows such an example and is structured analogously to fig. 4.25. A video of the full recording and corresponding DFMs are available online [77].

${ }^{17}$ Full activation of the tissue occurs from the $17^{\text {th }}$ pulse on. The frames $t_{9}$ and $t_{10}$ indicate this because no activity is visible and the last frame directly shows the full activation. 
This page was intentionally left blank. 


\section{Results}

The depicted example displays frames of a recording of a rabbit heart in VF during the application of a Syncrolation pulse sequence. The synchronization of the cardiac activity becomes apparent towards the end of the synchronizing pulses when similar activation sequences of the tissue occur after each pulse. This is demonstrated by the first two rows in fig. 4.26: Some activity exists near the apex just before a synchronizing pulse $\left(t_{0}\right)$ and spreads towards the base. The pulse then activates a large portion of the tissue, however some area is not activated (upper right, $t_{1}$ ). This area is activated afterwards but the activation cannot spread over the tissue that was activated by the pulse (compare $t_{2}$ and $t_{3}$ ). Instead some activation near the apex appears afterwards and spreads outward such that the activation pattern before the next pulse $\left(t_{4}\right)$ is similar to the one before the previous pulse $\left(t_{0}\right)$. The following activation $\left(t_{5}\right.$ to $\left.t_{8}\right)$ is very similar to the one just described. Thus, the activation sequences caused by the periodic pulses are almost stable/identical even though they change slightly from one pulse to the next.

In this example, the unpinning pulse leads to an activation of quite a large fraction of the tissue $\left(t_{9}\right)$. However, the activity is not stopped completely, but a large wave travels as a planar wave over the heart tissue afterwards $\left(t_{11}\right)$. This wave then breaks up to smaller waves and results in the continuation of the VF ( $t_{12}$ to $\left.t_{15}\right)$. The planar wave is an indication for the large degree of homogeneity of the activity. However, it might be that refractory tissue regions lead to the break up following the propagation of the large planar wave.

Figure 4.26 shows that the synchronizing pulse sequence changes the electric dynamics of the heart drastically: Instead of many small waves that propagate irregularly through the tissue, the pulses cause large homogeneous activations that propagate periodically along similar paths. This state will, however, typically not lead to the termination of $\mathrm{VF}$ as the wave propagation would continue and lead to wave break up when the periodic pulses stop. Thus, some additional control is required to transform the synchronized state into the steady state of no activity such that NSR can start again.

Syncrolation uses a high-energy, single shock for this transform. While in this example the defibrillation attempt fails and the VF continues anyways, a different timing of the unpinning pulse might have caused the termination of the VF (see also fig. 4.23). However, the design of Syncrolation originated from the insights gained on spiral unpinning in simulations. The dynamics that we observe on the surface of the heart during the application of Syncrolation, for example fig. 4.26, does not resemble the complex patterns that one might expect from multiple pinned spirals. Instead, large homogeneous activations are seen in the synchronized state. Even though it cannot be excluded that pinned spiral waves exist in the heart muscle and produce the observed activity, it is worth considering a different mechanism. An example is the one described above: Low amplitude pulses cause a synchronized state, widely control the dynamics and enforce regular activations which, however, do not suffice to terminate VF. Thus additional control is necessary to transition the regular state into the steady state. The last pulse of Syncrolation was designed to unpin spiral waves and therefore there is no reason why it should be effective in exercising the required control. 

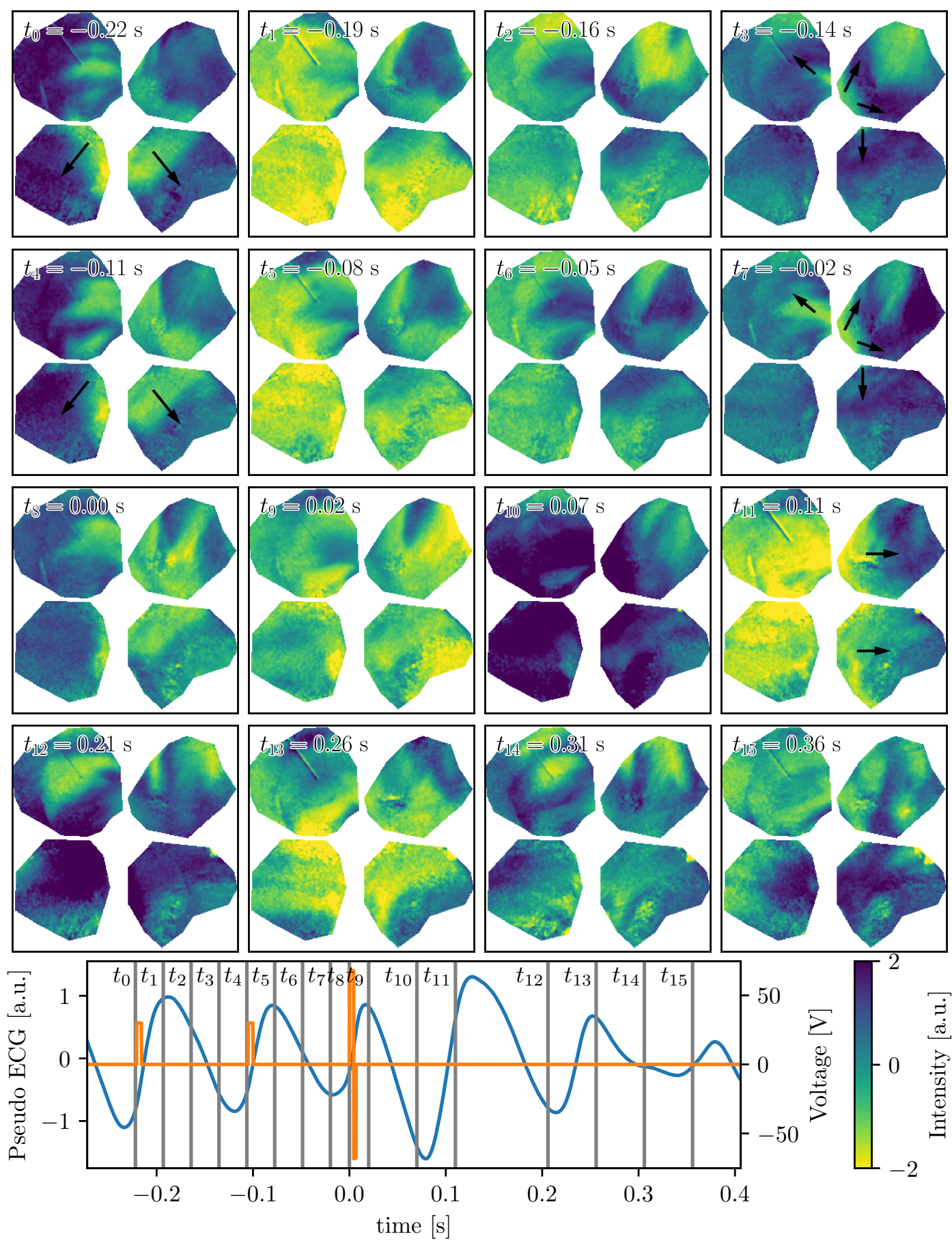

Figure 4.26: A defibrillation attempt of VF in a rabbit heart by Syncrolation is shown. The images display the optical mapping signal at various time points with yellow color indicating activation. The graph shows the Pseudo-ECG, a time series depicting the pulses and gray lines at times of the depicted frames with labels. For a detailed description and an interpretation please refer to the main text. 
I here presented an exemplary defibrillation attempt that shows a high synchronized area fraction towards the end of the periodic pulse sequence, but do not achieve simultaneous activation of the whole tissue. The last pulse of Syncrolation causes the propagation of a large, homogeneous planar wave, but does not terminate the arrhythmia. I suggested an interpretation of the observed activity, that attributes an increased order to the synchronized state which might allow an alternative defibrillation mechanism.

\subsubsection{Section Summary}

In section 4.1.5 I presented how measures like the maximum activated area or the synchronized area fraction depend on the frequency ratio $f_{p} / \overline{f_{d}}$ and on the voltage. In this section, I demonstrated that two different kinds of synchronized states can be observed. If we consider the synchronization as means for the termination of VF, these two states have different implications: Some periodic pulse sequences can synchronize the tissue enough such that VF is terminated, others create dynamics that are less complex and more homogeneous, which do not necessarily lead to termination of VF. In the latter case, some additional means of control is necessary for the termination.

This provides a new view on the Syncrolation method. This view suffices without the unpinning mechanism that originally led to the design of Syncrolation. This new view might in the future lead to modifications in the control strategy such as a replacement of the unpinning pulse in the end of the sequence. 


\section{Discussion}

In the previous chapter, I presented my experimental results and provided the interpretation that was necessary to follow the line of argumentation and the course of the analysis. In the following, I will further discuss those results and contrast them with the findings of other researchers. A condensed summary of the main results and conclusions will be provided in the next chapter.

\subsection{Simultaneous Unpinning}

A detailed discussion of my numerical work is included in the manuscript of the respective section (chapter 2). Briefly, my co-authors and I presented a novel method for simultaneous unpinning of multiple spiral waves and compare the new technique to an underdrive method which is advantageous in the case of a single pinned spiral. We also demonstrate which conditions are favorable for the new technique, e.g. which ranges of radii may exist in a system such that simultaneous unpinning is possible. The predicted superiority of the new method is validated in numerical simulations.

Notably, our findings contradict those of Hornung et al. [39] who predicted a $100 \%$ success rate for the underdrive method. While our study and theirs differ in multiple aspects, this discrepancy is most likely mainly due to the negligence of newly created pinned spiral waves in [39] which can counteract the unpinning.

On the one hand, the presented methodology used a generic model such that the results should not be restricted to a particular system. On the other hand, the electric dynamics of the heart during VF differ, in some regards, qualitatively from the dynamics of the investigated system. Therefore, it is unclear to what extent the findings are applicable when controlling cardiac dynamics.

\subsection{Syncrolation}

The role of pinned spiral waves during VF is still being discussed in literature and it might be that pinned spiral waves are essential for maintaining VF. Therefore, I conducted whole heart perfusion experiments in order to test whether a defibrillation technique inspired from the theoretical and numerical results on simultaneous unpinning, Syncrolation, is advantageous compared to conventional defibrillation.

An approach that is comparable to Syncrolation was presented by Pak et al. [63]: They used a feedback control system with four local electrodes before the application 


\section{Discussion}

of a specifically timed single shock for defibrillation. They showed, that the energy for $50 \%$ success rate of the single shock was reduced by about $10 \%$ compared to the defibrillation without preceding synchronization. The fundamental approach here is very similar to the one of Syncrolation: The complex dynamics of VF are altered by some initial control means to increase the defibrillation efficiency of a following single shock. The main differences to Syncrolation is that due to the local electrodes only a small region of the heart is influenced by the pacing. Also, the mechanistic understanding of Pak et al. [63] is different from the interpretations presented in this thesis (spiral unpinning and synchronized state transition; see below). They write that by timing the last shock such that the synchronized area is not excitable, "[...] the same shock cannot reinitiate VF in the low-voltage gradient area and results in successful defibrillation." [63]

An indication that a synchronized state could require a smaller energy for defibrillation is provided by Jin et al. [46]. They show that the DFT after 7 min since the onset of VF differs from DFT after $3 \mathrm{~min}$ in rabbit hearts. They only observe synchronized patterns in $\mathrm{VF}$ at times after the 3 min mark and postulate that this might cause the reduced $\mathrm{DFT}$ at $7 \mathrm{~min}$.

The multistage method by Li et al. [53] (see also section 1.5) should be mentioned here as they describe a defibrillation mechanism that also involves unpinning of spiral waves. However, please note that Li et al. [53] studied AF which differs considerably from VF which was considered in the work at hand. For example, the ventricles are much thicker allowing wave spreading in three dimensions compared to the quasi two-dimensional atria. Syncrolation uses multiple pulses to synchronize the tissue and a single unpinning pulse shall unpin spiral waves. Instead Li et al. [53] use multiple (unpinning) pulses in the first stage of their method to unpin wave fronts without preceding synchronization. Their second and third stage then shall prevent repinning and terminate remaining wave fronts [53]. If their mechanistic view was appropriate, it might not have been possible to show an energy reduction of Syncrolation compared to conventional single shock defibrillation because Syncrolation lacks means to prevent repinning and to terminate remaining waves.

The mechanistic motivation of Syncrolation, i.e. that spirals are entrained and then unpinned, directly creates some expectations of the results:

1. The success rate should depend on the delay $\tau$ as this delay determines whether the unpinning pulse is applied in the unpinning window.

2. If the initial pulse sequence synchronizes the tissue, the chance of defibrillation should be larger as spirals are entrained to allow simultaneous unpinning.

3. A pacing that is faster than the dynamics of the heart should have a higher success rate than one that is slower.

None of the above expectations were directly confirmed (see the following: 1.: figs. 4.21 and 4.22, 2.: fig. 4.24, 3. Hypo2 in section 4.2.6). However further analysis indicated that the success rate does depend on the ratio of the delay of the last pulse $\tau$ and the 
pacing period $T_{p}$ (see fig. 4.23) and that the success rate also depends on ratio of the pacing frequency $f_{p}$ and the dominant frequency ${\overline{f_{d}}}^{\text {opt }}$ (see fig. 4.20).

Thus, the overall outcome of the experiment series on Syncrolation did not match the expectations based on the mechanistic view involving unpinning of spiral waves. Furthermore, the dynamics that are observed on the heart surface (see section 4.3.2) do not resemble what would have been expected from pinned spiral waves nor are pinned spiral waves regularly seen on the heart surface ${ }^{1}$. Additionally, main features of VF, like the irregular activity, are not represented in the simulations of chapter 2 as it was pointed out before. Today, I therefore do not believe that a mechanistic view that is based on unpinning allows a better understanding of the here investigated dynamics of $\mathrm{VF}$ and its termination.

Nevertheless, I think that the experimentally collected data on Syncrolation and on synchronization is valuable in further understanding VF. I will demonstrate this in the following section.

\subsection{Synchronization}

In section 4.1, I analyzed quantitatively how the cardiac activity during VF is synchronized by periodic sequences of electric far field pulses. Few publications describe this synchronization process itself. Luther et al. [55] showed how the area that is activated by a pulse increases during the application of a LEAP sequence of low-energy far field pulses and Ji et al. [44] further showed that on average every pulse in a LEAP sequence causes a larger activated area than the previous one. However, even though the relevance of synchronization for the termination of VF is emphasized (especially in [44]), no data is provided how the synchronization depends on pulse parameters or the dynamics of the heart. I am also not aware of any other literature that provides such information. This gap is filled by the experimental data and the analysis that I presented in section 4.1 .

The average increase of the synchronized area fraction during VF due to a periodic pulse sequence shown in figs. 4.12 and 4.13 is in agreement with the above mentioned finding of an average increase of the activated area in [44]. They show that the average fraction of excited tissue increases with each shock.

Generally, figs. 4.12 and 4.13 show that the synchronized area fraction stays at a constant value after the initial increase until the end of the pulse sequence of typically 40 pulses. This would imply that the number of pulses in a synchronizing pulse sequence is not critical as long as enough pulses are applied, i.e. the plateau is reached. Only if the total energy that is used shall be minimized, the number of pulses will need to be reduced to the minimum that is necessary. However, some time series in fig. 4.12 might indicate that the synchronization measure does not stay on a constant

\footnotetext{
${ }^{1}$ Only in few cases, I did observe stationary spirals on the heart surface, but the dynamics were those of a VT in these cases. Also, it is not possible to judge whether they were only stationary or pinned.
} 


\section{Discussion}

level. A decrease of the synchronized area fraction after an early maximum would imply that synchronizing pulse sequences should not have more than the respective number of pulses.

While figs. 4.12 and 4.13 provide information on temporal evolution of the synchronization and thus on the length of synchronizing pulse sequences figs. 4.7 and 4.8 show how the synchronized area fraction and the maximum activated area depend on the voltage of the pulses and on the ratio of the pacing frequency and the dominant frequency of the VF. The results allow to predict what kind of synchronization can be expected for pulse sequences with certain voltage and frequency ratio and in particular demonstrates that pulse sequences with a frequency ratio $f_{p} / f_{d} \approx 1.1$ have the strongest synchronization for fixed voltages.

As described in section 1.4, electric far field shocks excite cardiac tissue at virtual electrodes near tissue heterogeneities. The field strength of the shocks determines near which heterogeneities the tissue is excited. At lower field strength fewer virtual electrodes exist and they are further separated in the tissue. If a virtual electrode periodically activates the tissue, then due to the properties of an excitable medium this activation will capture a larger region if it has a higher frequency than the surrounding activity $[90]^{2}$. However, I also pointed out before that heart tissue cannot be excited at an arbitrary rate. On the contrary, during VF often only small portions of the tissue are excitable and tissue which is excitable will typically be excited after a short time. Consider a tissue location near a virtual electrode. The periodic activation coming from the virtual electrode can only activate this tissue location when the activation wave arrives within this short time where the tissue is excitable. The period of the periodic excitation therefore should not be mush faster than the remaining activity because the activation wave would then arrive when the tissue is still refractory. Along these lines, periodic control will be more effective the faster it is as long as the period is larger than the (average) time that it takes the tissue after an activation to become excitable again. This argumentation does not only provide a possible explanation why the strongest synchronization is found for frequency ratios $f_{p} /{\overline{f_{d}}}^{\text {opt }}$ that are a bit greater than 1 . It also predicts that the frequency ratio for which this maximum is found depends on the time duration for which tissue is excitable during VF. The shorter this excitable time is, the closer the maximum will be to 1 .

\subsection{Termination through Synchronization}

In contrast to the few published findings on the synchronization process caused by periodic far field pulses if applied during VF, various publications exist that investigate techniques that are supposed to synchronize the tissue in order to terminate VF. This will be the focus of the following section.

\footnotetext{
${ }^{2} \mathrm{~A}$ similar argument was also presented in [55].
} 


\subsubsection{The Role of the Dominant Frequency}

When we assume that a termination of VF with techniques that synchronize the tissue is most likely when the synchronization is the strongest, then the results of section 4.1 allow to make predictions which pulse sequences should have the largest termination success rate. In particular, pulse sequences with a frequency ratio $f_{p} / \bar{f}_{d}{ }^{o p t} \approx 1.1$ were found to cause the strongest synchronization and should thus lead to the highest success rate. In the following, I will briefly describe the results of two publications that are based on numerical work and investigate defibrillation success of periodic pulse sequences depending on the pacing frequency of the sequence. Both publications relate the pacing frequency to the dominant frequency of the VF. I will relate my results to their findings after the initial description.

Ji et al. [44] provide numerical evidence using two-dimensional simulations employing Nygren and colleagues' atrial cell model, that a periodic pulse sequence with a period near the dominant frequency has the highest success rate in terminating VF with a small number of pulses. In particular, figure 8 in [44] shows that the success rate in their simulations is the highest around a pacing period of 1.1 times the dominant period. Note that, Ji et al. [44] use the most frequent value of the dominant frequencies of all pixels as the overall dominant frequency. Ji et al. [44] additionally provided a statistical analysis of the fraction of activated tissue in 26 defibrillation attempts with a periodic sequence of five far field pulses applied to 2 ventricles and 3 atria of mongrel dogs. The authors state that they observed successful defibrillation attempts using the periodic sequence of five pulse for deviations of about $15-25 \%$ from the dominant frequency.

Buran et al. [16] extensively studied the defibrillation success probabilities of a single pulse and periodic pulse series in two-dimensional excitable media with a distribution of circular heterogeneities using the Fenton-Karma model and the Luo-Rudy model. They studied the influence of the pacing period, electric field strength, and the number of pulses. They found that an optimal pacing period near the dominant frequency of the model had the largest reduction of the required energy. In the Fenton-Karma model, which has a broader power spectrum than the Luo-Rudy model, this dependency on the pacing period is less pronounced. The authors also show that the optimal pacing period is shifted towards larger periods when comparably large field strengths are used. Buran et al. [16] define the dominant frequency using an average power spectrum from 10 simulation of $10 \mathrm{~s}$ length, where the power spectra of all simulations and pixels are averaged.

In general, the results of $[44,16]$ and the ones presented in section 4.1 are in agreement in the sense that $[44,16]$ find the highest defibrillation success rates in their simulations for pacing frequencies that are near the dominant frequency of the VF and I demonstrated using experimental data that both the synchronized area fraction and the maximum activated area are the largest for pacing frequencies near the dominant frequency. However, the exact frequency ratio $f_{p} / f_{d}$ for which the largest success rate or the strongest synchronization is predicted differs. Ji et al. [44] present data where a ratio $f_{p} / f_{d}$ near 0.9 is best, Buran et al. [16] argue in favor of a ratio $f_{p} / f_{d}$ of 1 


\section{Discussion}

and in my results a ratio $f_{p} / f_{d} \approx 1.1$ corresponds to the strongest synchronization. This difference can be due to various reasons as the three studies differ in many aspects. Still, I pointed out in section 4.2 that the way how the dominant frequency is calculated can impact its absolute values. I thus think that it is crucial to consider what time window is used for the calculation (short window before the application of the shocks versus long time window of unperturbed dynamics), how the signal is measured (ECG versus optical) and whether the mean, the median or the peak of the histogram of dominant frequencies (in case of optical or simulation data) is used.

Likewise, the typical terms overdrive, underdrive and resonance often used in literature when describing pacing sequences should therefore be considered with care as they stem from a comparison of the pacing frequency with the dominant frequency which can be defined in various (sometimes not well documented) ways.

\subsubsection{Multisite Pacing}

Another approach to synchronize tissue and to facilitate defibrillation, that should be mentioned here, employs the local stimulation of heart tissue using multiple electrodes. Pak et al. [62] timed the electric stimulation with four local electrodes using a feedback loop coupled to the optical signal. The authors write that they aim at synchronizing the tissue in order to terminate VF. The highest VF termination success rate that they observed was $\approx 13 \%$ for a feedback mode where all four electrodes were commonly controlled according to the signal at a reference location.

In principal, the synchronization investigated by Pak et al. [62] is similar to the one studied in this thesis. While Pak et al. [62] use local electrodes for the control, in this thesis the heart is stimulated at many locations due to the virtual electrodes caused by the electric fields. Due to this access to a much larger portion if not the whole tissue is possible. A difference is the temporal sequence: I used a constant frequency that is set according to they dynamics before the pacing, while Pak et al. [62] present a real time technique without a fixed cycle length. Additionally, the use of local electrodes is a drawback in a potential clinical application.

\subsection{Mechanisms of Defibrillation}

The mechanism that leads to the termination of VF probably depends on the exercised control, i.e. the mechanism of conventional single shock defibrillation probably differs from the one with multiple low-energy pulses. Even though optical imaging techniques allow us nowadays to study the dynamics on the heart surface with high spatial and temporal resolution a full understanding of these mechanisms is still missing. This might for example be due to the complexity of activation during VF or due the missing information on the activity in the bulk tissue.

Nevertheless, various mechanisms have been proposed in literature and I offered corresponding interpretations in section 4.3. In the following, I will discuss my findings 
in the light of possible termination mechanisms and compare my interpretation to others.

The critical mass hypothesis predicts that a simultaneous depolarization of a sufficient fraction of the cardiac tissue will lead to successful termination of VF because not enough excitable tissue remains for fibrillatory waves to propagate into [88, 91]. While this theory was developed in the context of single shock defibrillation it might still apply for defibrillation with multiple pulses.

For example, Ji et al. [44] claim that in their work full phase synchronization of the tissue through multiple pulses leads to termination of VF. Also, Buran et al. [16] state that they find hints that synchronization might be the mechanism that leads to the defibrillation.

In section 4.3.1, I presented optical mapping data of a defibrillation with multiple pulses that had a much smaller voltage than the one typically necessary for single shock defibrillation. At the end of the pulse sequence the whole tissue (surface) was activated by the applied pulses. It seems plausible that this prevents further waves propagation and thereby terminates VF. In this view, this example can be seen as a support of the mechanism suggested in [44] and [16].

However, please note that there might be propagating activity in the tissue, especially in the septum, that would not be seen in the optical mapping recordings. This remaining activity could maintain VF and provides a plausible explanation why in some case pulses cause simultaneous activation of the whole heart surface but do not terminate VF (see also fig. 4.3). Furthermore, [25] already demonstrated in 1992 that high energy pulses also synchronize the repolarization of the tissue. I did not study the repolarization in the work at hand and it might well be that due to the reduced amplitude no synchronization of the repolarization occurs or is less pronounced, possibly causing a break up of a subsequent NSR activation. This could be the reason why a simultaneous activation of the heart surface is not a sufficient condition for termination of VF (see fig. 4.3).

Another defibrillation mechanism is suggested by Pak et al. [62] for their control with local electrodes. The authors suggest, that a "virtual reduction" of the tissue size through the synchronization caused by the pacing leads to an increased probability of terminating the VF. In the region covered by the electrodes the dynamics are less complex and thus the size of the tissue with complex dynamics is reduced. However, the region covered by the electrodes does still allow propagating signals in contrast to a real tissue size reduction which is known to promote self-termination [54, 32]. The example presented in section 4.3.2 shows how a very homogeneous activation after the application of Syncrolation again deteriorates into VF. Also, only pulse sequences with large maximum activated area values frequently caused termination of VF (see fig. 4.3) but high values of synchronized area fraction are found also for other parameters (compare fig. 4.7 and fig. 4.8). These results do not support the described virtual reduction mechanism VF.

In section 4.3.2, I described the dynamics of a Syncrolation defibrillation attempt where the synchronization lead to a regular, simple activation of the myocardium 
and I suggested that such a synchronization should be followed by a technique that exploits the simplified state to terminate the arrhythmia. In this view, the last shock of Syncrolation was such an attempt to transform the quite regular dynamics to the steady state, that was in this particular example unsuccessful (after the last shock a planar wave propagates over the heart surface and breaks up into VF). Tang et al. [75] present a similar approach, they state that "A failed near-threshold defibrillation shock is followed by an isoelectric window (IEW) and rapid repetitive responses that reinitiate ventricular fibrillation (VF)". They therefore applied a feedback protocol after a failed single shock defibrillation. However, the overall success rate was quite low (highest rate $<20 \%$ ). Apart from this, I am not aware of studies that investigate how a through control simplified cardiac activity could promote defibrillation.

It can be concluded that various ideas exist how defibrillation can be achieved. Experimental data and insights from simulations support some ideas and contradict others but so far no definite decision is possible which view provides the greatest benefit. Along these lines, the results of this thesis add a few pieces to this large puzzle. 


\section{Summary}

Ventricular fibrillation, a frequent cause of death, is a heart condition associated with complex patterns of electric activity on the heart surface. In spite of a century of research many aspects of how ventricular fibrillation is maintained or terminated remain unclear. One hypothesis is that rotating spiral waves of excitation pin to heterogeneities in the heart tissue, are thereby stabilized and maintain the fibrillation. Even though, extensive research was done on the control of single pinned spiral waves, little was previously known on how multiple spirals can be controlled and unpinned.

I filled this gap with my theoretical and numerical work using a simple model system where I presented a technique that allows simultaneous unpinning of multiple spiral waves. The proposed method is thereby considerably more effective than an underdrive method that is very effective in the case of single pinned spiral waves and that was suggested for unpinning of multiple spiral waves previously. I demonstrated that a problem that arises in systems with multiple heterogeneities is that electric far field pulses that are supposed to unpin spiral waves can create new pinned spiral waves. This problem is minimized with the proposed method that first synchronizes pinned spirals in order to allow simultaneous unpinning with another specifically timed pulse.

My colleagues and I designed a novel defibrillation method, Syncrolation, that is based on the same approach: A sequence of electric far field pulses synchronizes the heart tissue and an additional pulse unpins spiral waves and thereby terminates the ventricular fibrillation. In whole heart perfusion experiments with rabbit and pig hearts, I investigated the appropriate parameters of such a pulse sequence and finally compared the new method with conventional single shock defibrillation. Unfortunately, the new method could not be proven to be advantageous compared to conventional defibrillation. In contrast, many experimental results were unexpected such that the postulated mechanism of pinned spiral waves that maintain ventricular fibrillation is challenged.

Nevertheless, the investigation of Syncrolation and especially the basic research on the control via periodic pulse sequences provide exciting new insights. It was postulated in literature that periodic far field pulses synchronize the cardiac tissue and thereby terminate fibrillation. However, no experimental evidence was published on how this depends on parameters of the pulse sequence or on the dynamics in the heart. I consider it one of my main contributions that I provide comprehensive, quantitative, experimental evidence on control of cardiac activity via periodic electric far field pulses. In particular, I demonstrated that the synchronization strongly depends on the ratio of the pacing frequency and the dominant frequency of the ventricular fibrillation. 
Furthermore, I showed that the synchronized area fraction, a measure for the frequency synchronization, on average reaches a maximum after a comparably short time of 1 to $2 \mathrm{~s}$. This however again depends on the ratio of pacing frequency and dominant frequency of the ventricular fibrillation.

I believe that the findings presented in this thesis, especially the knowledge when synchronization of pulse sequences is strongest, contribute to the understanding of ventricular fibrillation and its control. This work supports future investigation and development of means to control and terminate fibrillating activity in the heart in general and of methods that employ periodic pulses to synchronize cardiac tissue in particular. 


\section{Bibliography}

[1] Multirecorder. URL http://www.bmp.ds.mpg.de/multirecorder.html. Accessed: 14.10 .2018 .

[2] F. Aguel, J. C. Eason, N. A. Trayanova, G. Iekas, and M. G. Fishler. Impact of Transvenous Lead Position on Active-Can ICD Defibrillation: A Computer Simulation Study. Pacing and Clinical Electrophysiology, 22(1):158-164, 1999. ISSN 1540-8159. doi: 10.1111/j.1540-8159.1999.tb00324.x.

[3] C. F. Babbs, W. A. Tacker, J. F. VanVleet, J. D. Bourland, and L. A. Geddes. Therapeutic indices for transchest defibrillator shocks: effective, damaging, and lethal electrical doses. American Heart Journal, 99(6):734-738, 1980.

[4] L. E. Bailey and S. D. Ong. Krebs-Henseleit solution as a physiological buffer in perfused and superfused preparations. Journal of Pharmacological Methods, 1(2): 171-175, 1978. ISSN 01605402. doi: 10.1016/0160-5402(78)90022-0.

[5] M. Bär and M. Eiswirth. Turbulence due to spiral breakup in a continuous excitable medium. Physical Review E, 48(3):R1635-R1637, 1993.

[6] D. Barkley. A model for fast computer simulation of waves in excitable media. Physica D Nonlinear Phenomena, 49:61-70, 1991.

[7] D. Barkley. Barkley model. Scholarpedia, 3(11):1877, 2008. ISSN 1941-6016. doi: 10.4249/scholarpedia.1877.

[8] A. Behrend, P. Bittihn, and S. Luther. Predicting unpinning success rates for a pinned spiral in an excitable medium. In Computing in Cardiology, pages 345-348, 2010 .

[9] R. M. Bell, M. M. Mocanu, and D. M. Yellon. Retrograde heart perfusion: The Langendorff technique of isolated heart perfusion. Journal of Molecular and Cellular Cardiology, 50(6):940-950, 2011. ISSN 0022-2828. doi: 10.1016/j.yjmcc. 2011.02.018.

[10] V. N Biktashev, A. V Holden, S. F Mironov, A. M Pertsov, and A. V Zaitsev. Three-dimensional organisation of re-entrant propagation during experimental ventricular fibrillation. Chaos, Solitons $\&$ Fractals, 13(8):1713-1733, 2002. ISSN 0960-0779. doi: 10.1016/S0960-0779(01)00164-3.

[11] P. Bittihn, G. Luther, E. Bodenschatz, V. Krinsky, U. Parlitz, and S. Luther. Far field pacing supersedes anti-tachycardia pacing in a generic model of excitable media. New Journal of Physics, 10(10):103012, 2008. 
[12] P. Bittihn, M. Hörning, and S. Luther. Negative Curvature Boundaries as Wave Emitting Sites for the Control of Biological Excitable Media. Physical Review Letters, 109(11), 2012. ISSN 0031-9007, 1079-7114. doi: 10.1103/PhysRevLett. 109.118106 .

[13] Philip Bittihn. Complex Structure and Dynamics of the Heart. PhD thesis, Georg-August Universität Göttingen, 2013.

[14] S. T. Buckland. Monte Carlo Methods For Confidence Interval Estimation Using The Bootstrap Technique. Journal of Applied Statistics, 10(2):194-212, 1983. ISSN 0266-4763, 1360-0532. doi: 10.1080/02664768300000017.

[15] Statistisches Bundesamt. Gesundheit - Todesursachen in Deutschland - 2015. 2017.

[16] P. Buran, M. Bär, S. Alonso, and T. Niedermayer. Control of electrical turbulence by periodic excitation of cardiac tissue. Chaos, 27(11):113110, 2017. ISSN 10541500. doi: 10.1063/1.5010787.

[17] J. Caldwell, F. L. Burton, G. L. Smith, and S. M. Cobbe. Heterogeneity of Ventricular Fibrillation Dominant Frequency During Global Ischemia in Isolated Rabbit Hearts. Journal of Cardiovascular Electrophysiology, 18(8):854-861, 2007. ISSN 1540-8167. doi: 10.1111/j.1540-8167.2007.00867.x.

[18] B. Choi, W. Nho, and G. Liu, T. Salama. Life Span of Ventricular Fibrillation Frequencies. Circulation Research, 91(4):339-345, 2002. ISSN 0009-7330, 15244571. doi: 10.1161/01.RES.0000031801.84308.F4.

[19] J. Christoph, J. Schröder-Schetelig, and S. Luther. Electromechanical optical mapping. Progress in Biophysics and Molecular Biology, 130:150-169, 2017. ISSN 0079-6107. doi: 10.1016/j.pbiomolbio.2017.09.015.

[20] T. Church, M. Martinson, M. Kallok, and W. Watson. A Model to Evaluate Alternative Methods of Defibrillation Threshold Determination. Pacing and Clinical Electrophysiology, 11(11):2002-2007, 1988. ISSN 1540-8159. doi: 10.1111/ j.1540-8159.1988.tb06341.x.

[21] R. H. Clayton, O. Bernus, E. M. Cherry, H. Dierckx, F. H. Fenton, L. Mirabella, A. V. Panfilov, F. B. Sachse, G. Seemann, and H. Zhang. Models of cardiac tissue electrophysiology: Progress, challenges and open questions. Elsevier Ltd., 104:22-48, 2011.

[22] A. Connolly, E. Vigmond, and M. Bishop. Virtual electrodes around anatomical structures and their roles in defibrillation. PLOS ONE, 12(3):e0173324, 2017. ISSN 1932-6203. doi: 10.1371/journal.pone.0173324.

[23] J. M. Davidenko, A. V. Pertsov, R. Salomonsz, W. Baxter, and J. Jalife. Stationary and drifting spiral waves of excitation in isolated cardiac muscle. Nature, 355 (6358):349-351, 1992. doi: 10.1038/355349a0. 
[24] J. Davy, E. S. Fain, P. Dorian, and R. A. Winkle. The relationship between successful defibrillation and delivered energy in open-chest dogs: reappraisal of the "defibrillation threshold" concept. American Heart Journal, 113(1):77-84, 1987.

[25] S. M. Dillon. Synchronized repolarization after defibrillation shocks. A possible component of the defibrillation process demonstrated by optical recordings in rabbit heart. Circulation, 85(5):1865-1878, 1992.

[26] W. J. Dixon. The Up-and-Down Method for Small Samples. Journal of the American Statistical Association, 60(312):967, 1965. ISSN 01621459. doi: 10. $2307 / 2283398$.

[27] I. R. Efimov, V. P. Nikolski, and G. Salama. Optical Imaging of the Heart. Circulation Research, 95(1):21-33, 2004. ISSN 0009-7330, 1524-4571. doi: 10. 1161/01.RES.0000130529.18016.35.

[28] B. Efron. Bootstrap methods: Another look at the jackknife. The Annals of Statistics, 7(1):1-26, 1979.

[29] F. H. Fenton, S. Luther, E. M. Cherry, N. F. Otani, V. Krinsky, A. Pumir, E. Bodenschatz, and R. F. Gilmour. Termination of Atrial Fibrillation Using Pulsed Low-Energy Far-Field Stimulation. Circulation, 120(6):467-476, 2009. ISSN 0009-7322, 1524-4539. doi: 10.1161/CIRCULATIONAHA.108.825091.

[30] T. Fitschen, D. Hornung, A. Schlemmer, and H. tom Wörden. CaosDB, October 2018. URL https://gitlab.gwdg.de/bmp-caosdb. http://dx.doi.org/10.17617/3.1s.

[31] T. Fitschen, A. Schlemmer, D. Hornung, H. tom Wörden, U. Parlitz, and S. Luther. Caosdb - research data management for complex, changing, and automated research workflows. CoRR, abs/1801.07653, 2018. URL http://arxiv.org/abs/ 1801.07653.

[32] W. E. Garrey. The nature of fibrillary contraction of the heart.-its relation to tissue mass and form. American Journal of Physiology-Legacy Content, 33(3): 397-414, 1914.

[33] R. A. Gray and J. P. Wikswo. Cardiovascular disease: Several small shocks beat one big one. Nature, 475(7355):181-182, 2011. ISSN 0028-0836. doi: 10.1038/475181a.

[34] R. A. Gray, A. M. Pertsov, and J. Jalife. Spatial and temporal organization during cardiac fibrillation. Nature, 392(6671):75-78, 1998. ISSN 0028-0836. doi: $10.1038 / 32164$.

[35] T. J. Herron, P. Lee, and J. Jalife. Optical Imaging of Voltage and Calcium in Cardiac Cells \& Tissues. Circulation Research, 2012. 
[36] J. Heuser. 2007. URL http://commons.wikimedia.org/wiki/File:RLS_ 12blauLeg.png. Published under Creative Commons Attribution 2.5 Generic License: http://creativecommons.org/licenses/by/2.5/; based on original work by Patrick J. Lynch, medical illustrator and C. Carl Jaffe, MD, cardiologist.

[37] R. Hinkel, C. El-Aouni, T. Olson, J. Horstkotte, S. Mayer, S. Müller, M. Willhauck, C. Spitzweg, F. Gildehaus, W. Münzing, E. Hannappel, I. Bock-Marquette, J. M. DiMaio, A. K. Hatzopoulos, P. Boekstegers, and C. Kupatt. Thymosin $\beta 4$ Is an Essential Paracrine Factor of Embryonic Endothelial Progenitor Cell-Mediated Cardioprotection. Circulation, 117(17):2232-2240, 2008. ISSN 0009-7322, 15244539. doi: 10.1161/CIRCULATIONAHA.107.758904.

[38] D. Hornung. Cardiac Arrhythmia Termination on the Vascular and Organ Scale. PhD thesis, Georg-August Universität Göttingen, 2014.

[39] D. Hornung, V. N. Biktashev, N. F. Otani, T. K. Shajahan, T. Baig, S. Berg, S. Han, V. I. Krinsky, and S. Luther. Mechanisms of vortices termination in the cardiac muscle. Royal Society Open Science, 4(3):170024, 2017. ISSN 2054-5703. doi: $10.1098 /$ rsos.170024.

[40] V. D. Hosfeld, S. Puwal, K. Jankowski, and B. J. Roth. A Model for Multi-site Pacing of Fibrillation Using Nonlinear Dynamics Feedback. Journal Of Biological Physics, 33(2):145-153, 2007. ISSN 1573-0689. doi: 10.1007/s10867-007-9049-9.

[41] M. Hörning. Termination of pinned vortices by high-frequency wave trains in heartlike excitable media with anisotropic fiber orientation. Physical Review E, 86(3):031912, 2012. doi: 10.1103/PhysRevE.86.031912.

[42] J. Jalife. Ventricular Fibrillation: Mechanisms of Initiation and Maintenance. Annual Review of Physiology, 62(1):25-50, 2000. doi: 10.1146/annurev.physiol. 62.1.25.

[43] J. Jalife. Déjà vu in the theories of atrial fibrillation dynamics. Cardiovascular Research, 89(4):766-775, 2011. ISSN 0008-6363. doi: 10.1093/cvr/cvq364.

[44] Y. C. Ji, I. Uzelac, N. F. Otani, S. Luther, R. F. Gilmour, E. M. Cherry, and F. H. Fenton. Synchronization as a Mechanism for Low-energy Anti-fibrillation Pacing (LEAP). Heart Rhythm, 2017. ISSN 15475271. doi: 10.1016/j.hrthm.2017.05.021.

[45] D. Jin, J. Wang, K. Yang, K. Wang, W. Quan, U. Herken, and Y. Li. A Grouped Up-and-Down Method Used for Efficacy Comparison Between Two Different Defibrillation Waveforms. IEEE Transactions on Biomedical Engineering, 63(2): 385-391, February 2016. ISSN 0018-9294. doi: 10.1109/TBME.2015.2458976.

[46] Q. Jin, J. Zhou, N. Zhang, C. Lin, Y. Pang, Y. Xin, J. Pan, G. Gu, W. Shen, and L. Wu. Defibrillation Threshold Varies During Different Stages of Ventricular Fibrillation in Canine Hearts. Heart, Lung and Circulation, 22(2):133-140, February 2013. ISSN 1443-9506. doi: 10.1016/j.hlc.2012.08.059. 
[47] H. C. M. Kamphuis, J. R. J. de Leeuw, R. Derksen, R. N. W. Hauer, and J. A. M. Winnubst. Implantable cardioverter defibrillator recipients: quality of life in recipients with and without ICD shock delivery. EP Europace, 5(4):381-389, 2003. ISSN 1099-5129. doi: 10.1016/S1099-5129(03)00078-3.

[48] V. I. Krinsky and K. I. Agladze. Interaction of rotating waves in an active chemical medium. Physica D: Nonlinear Phenomena, 8(1-2):50-56, 1983. ISSN 0167-2789. doi: 10.1016/0167-2789(83)90310-X.

[49] C. Kupatt. Selective retroinfusion of GSH and cariporide attenuates myocardial ischemia-reperfusion injury in a preclinical pig model. Cardiovascular Research, 61(3):530-537, 2004. ISSN 00086363. doi: 10.1016/j.cardiores.2003.11.012.

[50] K. F. Kwaku and S. M. Dillon. Shock-Induced Depolarization of Refractory Myocardium Prevents Wave-Front Propagation in Defibrillation. Circulation Research, 79(5):957-973, 1996. ISSN 0009-7330, 1524-4571. doi: 10.1161/01.RES. 79.5.957.

[51] J. I. Laughner, F. S. Ng, M. S. Sulkin, R. M. Arthur, and I. R. Efimov. Processing and analysis of cardiac optical mapping data obtained with potentiometric dyes. American Journal of Physiology-Heart and Circulatory Physiology, 303(7):H753H765, 2012. ISSN 0363-6135. doi: 10.1152/ajpheart.00404.2012.

[52] W. Li, C. M. Ripplinger, Q. Lou, and I. R. Efimov. Multiple monophasic shocks improve electrotherapy of ventricular tachycardia in a rabbit model of chronic infarction. Heart Rhythm, 6(7):1020-1027, 2009. ISSN 15475271. doi: 10.1016/j.hrthm.2009.03.015.

[53] W. Li, A. H. Janardhan, V. V. Fedorov, Q. Sha, R. B. Schuessler, and I. R. Efimov. Low-Energy Multistage Atrial Defibrillation Therapy Terminates Atrial Fibrillation With Less Energy Than a Single Shock. Circulation:Arrhythmia and Electrophysiology, 4(6):917-925, 2011. ISSN 1941-3149, 1941-3084. doi: 10.1161/CIRCEP.111.965830.

[54] T. Lilienkamp, J. Christoph, and U. Parlitz. Features of chaotic transients in excitable media governed by spiral and scroll waves. Phys. Rev. Lett., 119:054101, Jul 2017. doi: 10.1103/PhysRevLett.119.054101.

[55] S. Luther, F. H. Fenton, B. G. Kornreich, A. Squires, P. Bittihn, D. Hornung, M. Zabel, J. Flanders, A. Gladuli, L. Campoy, et al. Low-energy control of electrical turbulence in the heart. Nature, 475(7355):235-239, 2011.

[56] R. A. Malkin, J. M. Herre, L. McGowen, M. M. Tenzer, J. R. Onufer, N. J. Stamato, M. Wood, and R. C. Bernstein. A Four-Shock Bayesian Up-Down Estimator of the $80 \%$ Effective Defibrillation Dose. Journal of Cardiovascular Electrophysiology, 10(7):973-980, 1999. ISSN 1540-8167. doi: 10.1111/j.1540-8167. 1999.tb01268.x.

[57] A. G. Mayor. Rhythmical pulsation in scyphomedusae. (47), 1906. 
[58] W. C. McDaniel and J. C. Schuder. An Up-Down Algorithm for Estimation of the Cardiac Ventricular Defibrillation Threshold. Medical Instrumentation, 22(6): 286-292, 1988.

[59] J. M. Meunier, S. Ramalingam, S. Lin, and A. R. Patwardhan. Capture of activation during ventricular arrhythmia using distributed stimulation. Journal Of Interventional Cardiac Electrophysiology, 18(3):207-215, 2007. ISSN 1572-8595. doi: 10.1007/s10840-007-9094-0.

[60] G. R. Mines. On circulating excitations in heart muscles and their possible relation to tachycardia and fibrillation. Transactions Of The Royal Society Of Canada.

[61] K. Nanthakumar, P. L. Johnson, J. Huang, C. R. Killingsworth, D. L. Rollins, H. T. McElderry, W. M. Smith, and R. E. Ideker. Regional Variation in Capture of Fibrillating Swine Left Ventricle During Electrical Stimulation. Journal of Cardiovascular Electrophysiology, 16(4):425-432, 2005. ISSN 1540-8167. doi: 10.1046/j.1540-8167.2005.40517.x.

[62] H. Pak, Y. Liu, H. Hayashi, Y. Okuyama, P. Chen, and S. Lin. Synchronization of ventricular fibrillation with real-time feedback pacing: implication to low-energy defibrillation. American Journal of Physiology - Heart and Circulatory Physiology, 285(6):H2704-H2711, 2003. ISSN 0363-6135, 1522-1539. doi: 10.1152/ajpheart. 00366.2003.

[63] H. Pak, Y. Okuyama, Y.-Seog Oh, H.ki Hayashi, Y. Liu, P. Chen, and S. Lin. Improvement of Defibrillation Efficacy with Preshock Synchronized Pacing. Journal of Cardiovascular Electrophysiology, 15(5):581-587, 2004. ISSN 1540-8167. doi: 10.1046/j.1540-8167.2004.03573.x.

[64] D. Pan, X.d Pan J. Gao, X.and Feng, and H. Zhang. Removal of pinned scroll waves in cardiac tissues by electric fields in a generic model of threedimensional excitable media. Scientific Reports, 6:21876, 2016. ISSN 2045-2322. doi: $10.1038 /$ srep21876.

[65] S. V. Pandit and J. Jalife. Rotors and the Dynamics of Cardiac Fibrillation. Circulation Research, 112(5):849-862, 2013. ISSN 0009-7330, 1524-4571. doi: 10.1161/CIRCRESAHA.111.300158.

[66] R. Plonsey and R. C. Barr. Effect of microscopic and macroscopic discontinuities on the response of cardiac tissue to defibrillating (stimulating) currents. Medical ES Biological Engineering \& Computing, 24(2):130-136, 1986. ISSN 0140-0118, 1741-0444. doi: 10.1007/BF02443925.

[67] A. Pumir, V. Nikolski, M. Hörning, A. Isomura, K. Agladze, K. Yoshikawa, R. Gilmour, E. Bodenschatz, and V. Krinsky. Wave Emission from Heterogeneities Opens a Way to Controlling Chaos in the Heart. Physical Review Letters, 99(20), 2007. ISSN 0031-9007, 1079-7114. doi: 10.1103/PhysRevLett.99.208101. 
[68] Z. Qu and J. N. Weiss. Mechanisms of Ventricular Arrhythmias: From Molecular Fluctuations to Electrical Turbulence. Annual Review of Physiology, 77(1):29-55, 2015. ISSN 0066-4278. doi: 10.1146/annurev-physiol-021014-071622.

[69] M. F. Rattes, D. L. Jones, A. D. Sharma, and G. J. Klein. Defibrillation Threshold: A Simple and Quantitative Estimate of the Ability to Defibrillate. Pacing and Clinical Electrophysiology, 10(1):70-77, 1987. ISSN 1540-8159. doi: 10.1111/j.1540-8159.1987.tb05926.x.

[70] C. M. Ripplinger, V. I. Krinsky, V. P. Nikolski, and I. R. Efimov. Mechanisms of unpinning and termination of ventricular tachycardia. American Journal of Physiology - Heart and Circulatory Physiology, 291(1):H184-H192, 2006. ISSN 0363-6135, 1522-1539. doi: 10.1152/ajpheart.01300.2005.

[71] R. Rosenthal. Combining results of independent studies. Psychological Bulletin, 85(1):185-193, 1978. ISSN 1939-1455(Electronic),0033-2909(Print). doi: 10.1037/ 0033-2909.85.1.185.

[72] S. Sawai, P. A. Thomason, and E. C. Cox. An autoregulatory circuit for longrange self-organization in Dictyostelium cell populations. Nature, 433(7023): 323-326, 2005. ISSN 1476-4687. doi: 10.1038/nature03228.

[73] A. Schlemmer. Intermittent Complexity Fluctuations during Ventricular Fibrillation. PhD thesis, Georg-August Universität Göttingen, 2017.

[74] E. B. Schron, D. V. Exner, Q. Yao, L. S. Jenkins, J. S. Steinberg, J. R. Cook, S. P. Kutalek, P. L. Friedman, R. S. Bubien, R. L. Page, and J. Powell. Quality of life in the antiarrhythmics versus implantable defibrillators trial: impact of therapy and influence of adverse symptoms and defibrillator shocks. Circulation, 105(5):589-594, 2002. ISSN 0009-7322. doi: 10.1161/hc0502.103330.

[75] L. Tang, G. Hwang, P. Song, J. Chen, and S. Lin. Post-Shock Synchronized Pacing in Isolated Rabbit Left Ventricle: Evaluation of a Novel Defibrillation Strategy. Journal of Cardiovascular Electrophysiology, 18(7):740-749, 2007. ISSN 1540-8167. doi: 10.1111/j.1540-8167.2007.00792.x.

[76] H. tom Wörden. Synchronization by periodic pulses during ventricular fibrillation, 2018. URL https://doi.org/10.25625/AIRJT1.

[77] H. tom Wörden. Vf termination, 2018. URL https://doi.org/10.25625/ X3XOQF.

[78] H. tom Wörden, D. Hornung, and T. Baig. Apparatus for terminating or unpinning rotating electric activity in a cardiac tissue, 2017. EP 3342455.

[79] N. A. Trayanova, B. J. Roth, and L. J. Malden. The response of a spherical heart to a uniform electric field: a bidomain analysis of cardiac stimulation. IEEE Transactions on Biomedical Engineering, 40(9):899-908, 1993. ISSN 0018-9294. doi: 10.1109/10.245611. 
[80] K. Umapathy, S. Masse, E. Sevaptsidis, J. Asta, H. Ross, N. Thavandiran, K. Nair, T. Farid, R. Cusimano, J. Rogers, S. Krishnan, and K. Nanthakumar. Regional frequency variation during human ventricular fibrillation. Medical Engineering \&6 Physics, 31(8):964-970, 2009. ISSN 1350-4533. doi: 10.1016/j.medengphy.2009. 05.009 .

[81] M. Valderrabano. Frequency Analysis of Ventricular Fibrillation in Swine Ventricles. Circulation Research, 90(2):213-222, 2002. ISSN 00097330, 15244571. doi: 10.1161/hh0202.103645.

[82] F. Varghese, J. U. Neuber, F. Xie, J. M. Philpott, A. G. Pakhomov, and C. W. Zemlin. Low-energy defibrillation with nanosecond electric shocks. Cardiovasc. Res., 113(14):1789-1797, December 2017. ISSN 0008-6363. doi: 10.1093/cvr/ cvx172. WOS:000417338500015.

[83] G. P. Walcott, C. R. Killingsworth, and R. E. Ideker. Do clinically relevant transthoracic defibrillation energies cause myocardial damage and dysfunction? Resuscitation, 59(1):59-70, 2003. ISSN 0300-9572. doi: 10.1016/S0300-9572(03) 00161-8.

[84] S. Weidmann. Effect of current flow on the membrane potential of cardiac muscle. The Journal of Physiology, 115(2):227-236. doi: 10.1113/jphysiol.1951.sp004667.

[85] D. Wilson and J. Moehlis. An Energy-Optimal Methodology for Synchronization of Excitable Media. SIAM Journal On applied Dynamical Systems, 13(2):944-957, 2014. doi: $10.1137 / 130942851$.

[86] A. T. Winfree. Spiral Waves of Chemical Activity. Science, 175(4022):634-636, 1972. ISSN 0036-8075, 1095-9203. doi: 10.1126/science.175.4022.634.

[87] A. T. Winfree. Electrical turbulence in three-dimensional heart muscle. Science, 266(5187):1003-1006, 1994. ISSN 0036-8075, 1095-9203. doi: 10.1126/science. 7973648 .

[88] M. Yashima, Y. Kim, S. Armin, T. Wu, Y. Miyauchi, W. J. Mandel, P. Chen, and H. S. Karagueuzian. On the mechanism of the probabilistic nature of ventricular defibrillation threshold. American Journal of Physiology-Heart and Circulatory Physiology, 284(1):H249-H255, January 2003. ISSN 0363-6135. doi: 10.1152/ajpheart.00742.2002.

[89] H. Zhang, K. Iijima, J. Huang, G. P. Walcott, and J. M. Rogers. Optical Mapping of Membrane Potential and Epicardial Deformation in Beating Hearts. Biophysical Journal, 111(2):438-451, 2016. ISSN 0006-3495. doi: 10.1016/j.bpj.2016.03.043.

[90] D. P. Zipes. Cardiac Electrophysiology. Elsevier, 2004. ISBN 978-0-7216-0323-0. doi: 10.1016/B0-7216-0323-8/X5001-5.

[91] D. P. Zipes, J. Fischer, R. M. King, A. Nicoll, and W. W. Jolly. Termination of ventricular fibrillation in dogs by depolarizing a critical amount of myocardium. The American Journal of Cardiology, 36, 1975. 


\section{A Appendix}

\section{A.1 Abbreviations}

AF Atrial Fibrillation

BMPG Biomedical Physics Group

DFM Dominant Frequency Map

ECG Electrocardiogram

ICD Implantable Cardioverter-Defibrillators

LV Left Ventricle

NSR Normal Sinus Rhythm

RV Right Ventricle

VF Ventricular Fibrillation

\section{A.2 Acknowledgement}

This research has received funding from the Deutsche Forschungsgemeinschaft (SFB 937 Collective Behavior of Soft and Biological Matter, project A18; SFB 1002 Modulatory Units in Heart Failure, project C03).

I would like to thank:

- Stefan Luther and Ulrich Parlitz for their excellent support and advice.

- Daniel Hornung for teaching me experimental procedures.

- Daniel Hornung, Sebastian Berg, Alexander Schlemmer and many more for conducting or assisting with experiments.

- Alexander Schlemmer, Daniel Hornung, Sebastian Berg and the whole work group for fruitful discussions and a great working atmosphere.

- Timm Fitschen for frequent support with CaosDB.

- Sebastian Berg, Alexander Schlemmer, Daniel Hornung, Tariq Baig and many more for sharing source code. 
- All people contributing to open source software that I used and especially the authors of PythonAnalyser, MultiRecorder, Pulsar and CaosDB since my work would not have been possible without those numerous open source tools.

\section{A.3 Competing Interests}

I am one of three applicants for the "EXIST Gründerstipendium" of the Federal Ministry for Economic Affairs and Energy. We applied for support to allow the founding of a company that offers services in the context of the CaosDB software.

\section{A.4 Curriculum Vitae}

18.07.1988 | born in Stade

2008 Abitur at the Vincent-Lübeck-Gymnasium Stade

2008/2009 Civil Service at the Institut für Solarenergieforschung in Hameln

2012 B. Sc. at the Georg-August-Universität Göttingen

2013 Semester abroad at the University of Auckland, Neuseeland

2014 M. Sc. at the Georg-August-Universität Göttingen

2018 Dr. rer. nat. at the Georg-August-Universität Göttingen (expected)

\section{A.5 Figures}



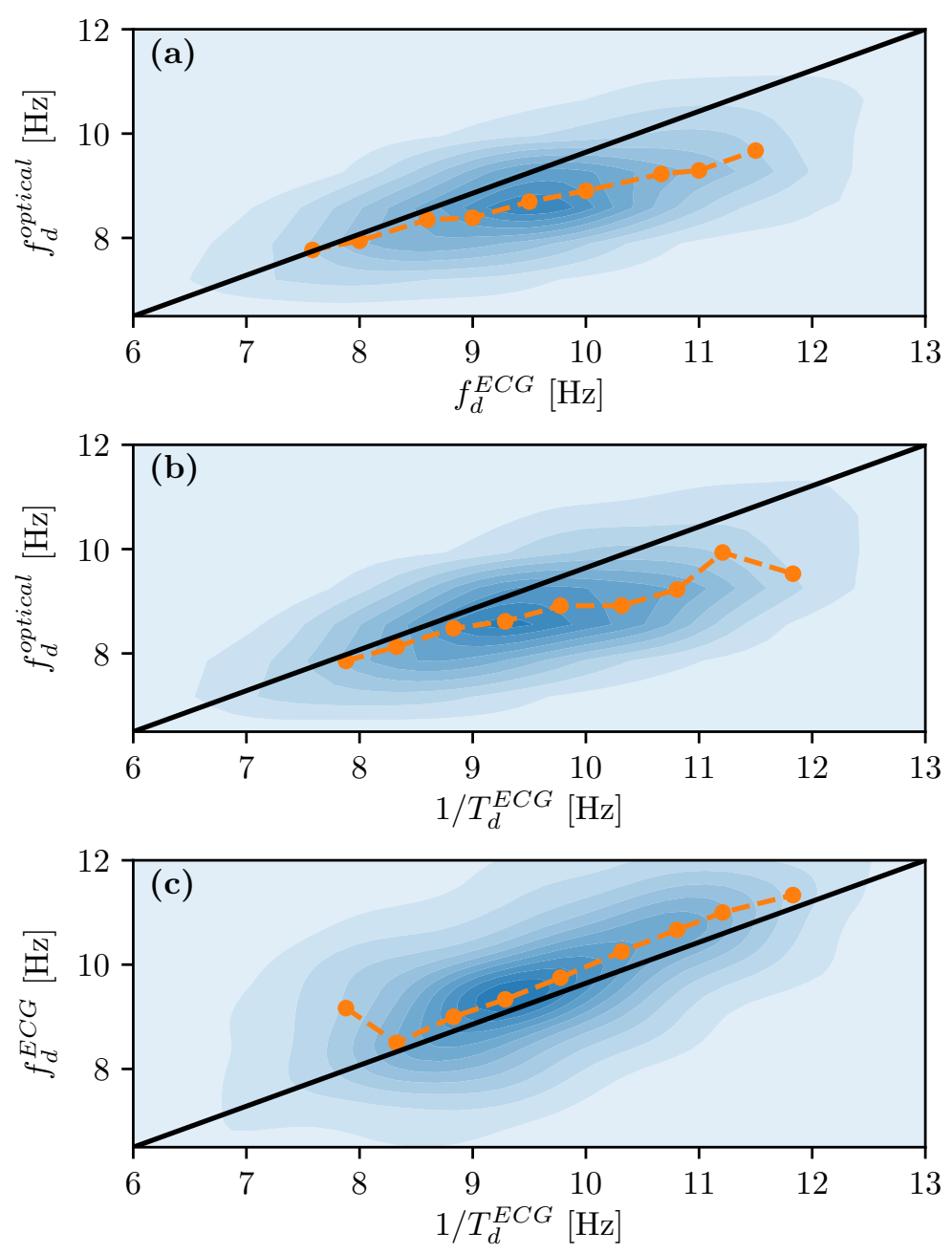

Figure A.1: Different measures of the VF frequency are plotted against each other. This plot illustrates that measures from the ECG differ from the one from the optical data. For every defibrillation attempt with Syncrolation recorded from a rabbit heart, three measures are calculated to characterize the frequency of the VF before the attempt: the mean dominant frequency of the optical data $\overline{f_{d}}{ }^{o p t}$, the dominant frequency of the ECG $f_{d}^{E C G}$ and the frequency calculated from the period that was calculated on the fly in the experiment using a sine fitting algorithm. Each diagram shows the data corresponding to a pair of those measures. The color coded background depicts a kernel density estimation of the data. The black line is the identity and the orange data points are the median values of both measures for bins along the abscissa. Diagrams (a) and (b) show that the measure ${\overline{f_{d}}}^{\text {opt }}$ deviates from the measures from the ECG. (c), however, illustrates that the two measures from the ECG are very similar. 

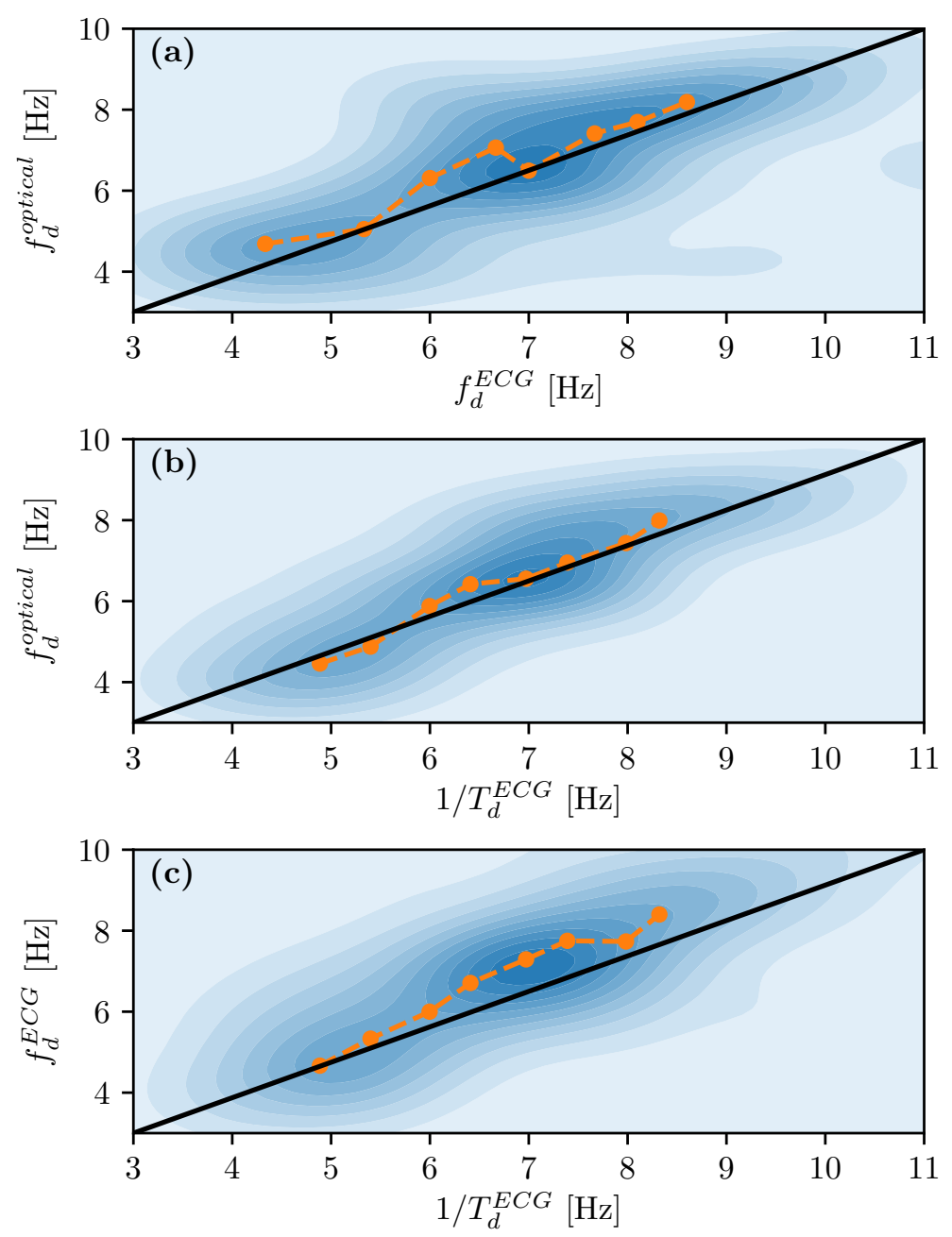

Figure A.2: Identical to fig. A.1 except that data from experiments with pig hearts is shown. See the caption of fig. A.1 for details. Diagrams (a) and (b) do not show a considerable difference between the measure ${\overline{f_{d}}}^{\text {opt }}$ and measures from the ECG. (c) however indicates a small difference between the two measures from the ECG. 

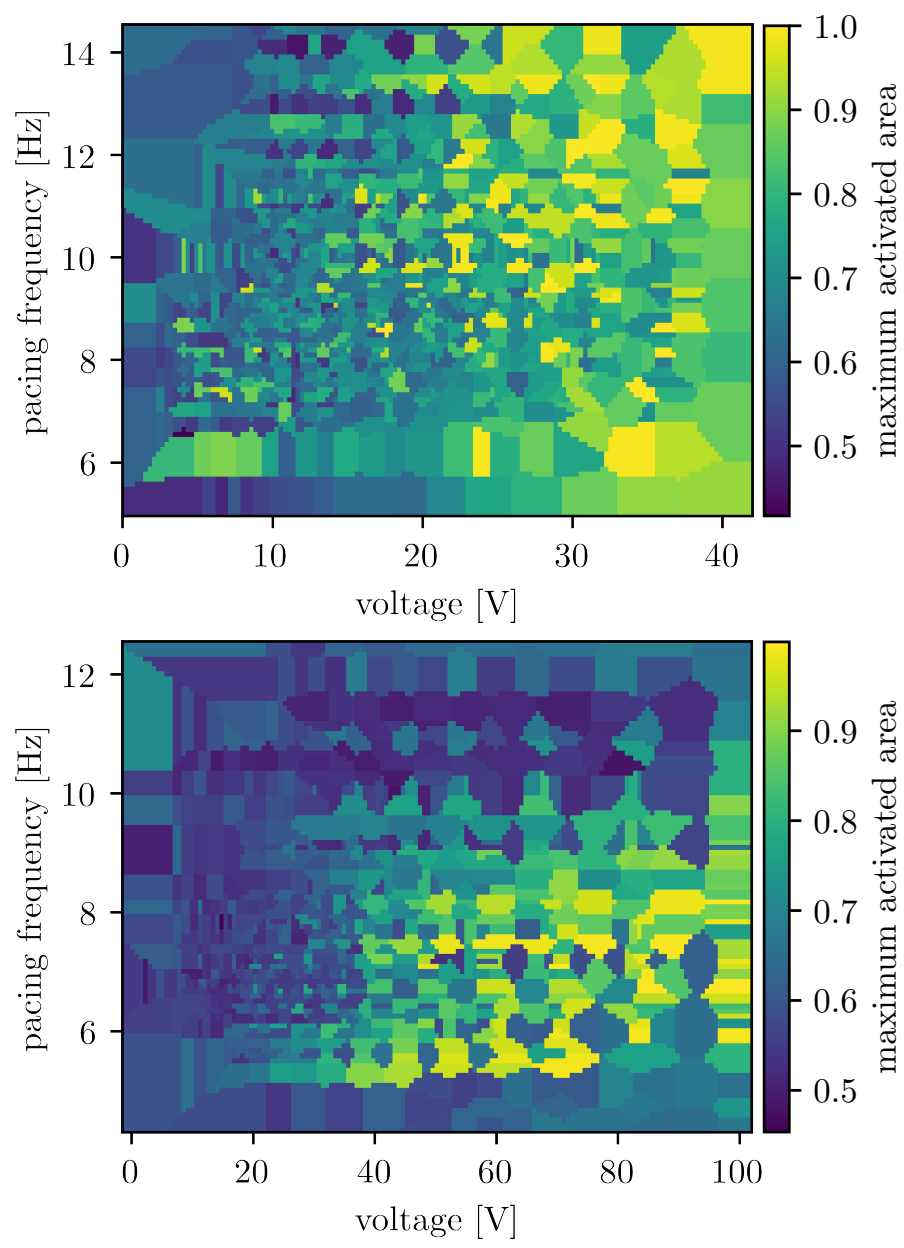

Figure A.3: Maximum activated area caused by periodic pulses during VF. The diagram is analogous to the corresponding diagrams in fig. 4.4. However, here nearest neighbor interpolation was used in order to illustrate that close by parameter values can lead to very different maximum activated area fractions. This is the reason for the averaging that was applied in fig. 4.4. The upper (lower) diagram depicts data from experiments with rabbit (pig) hearts. Please see the caption of fig. 4.4 for further details. 

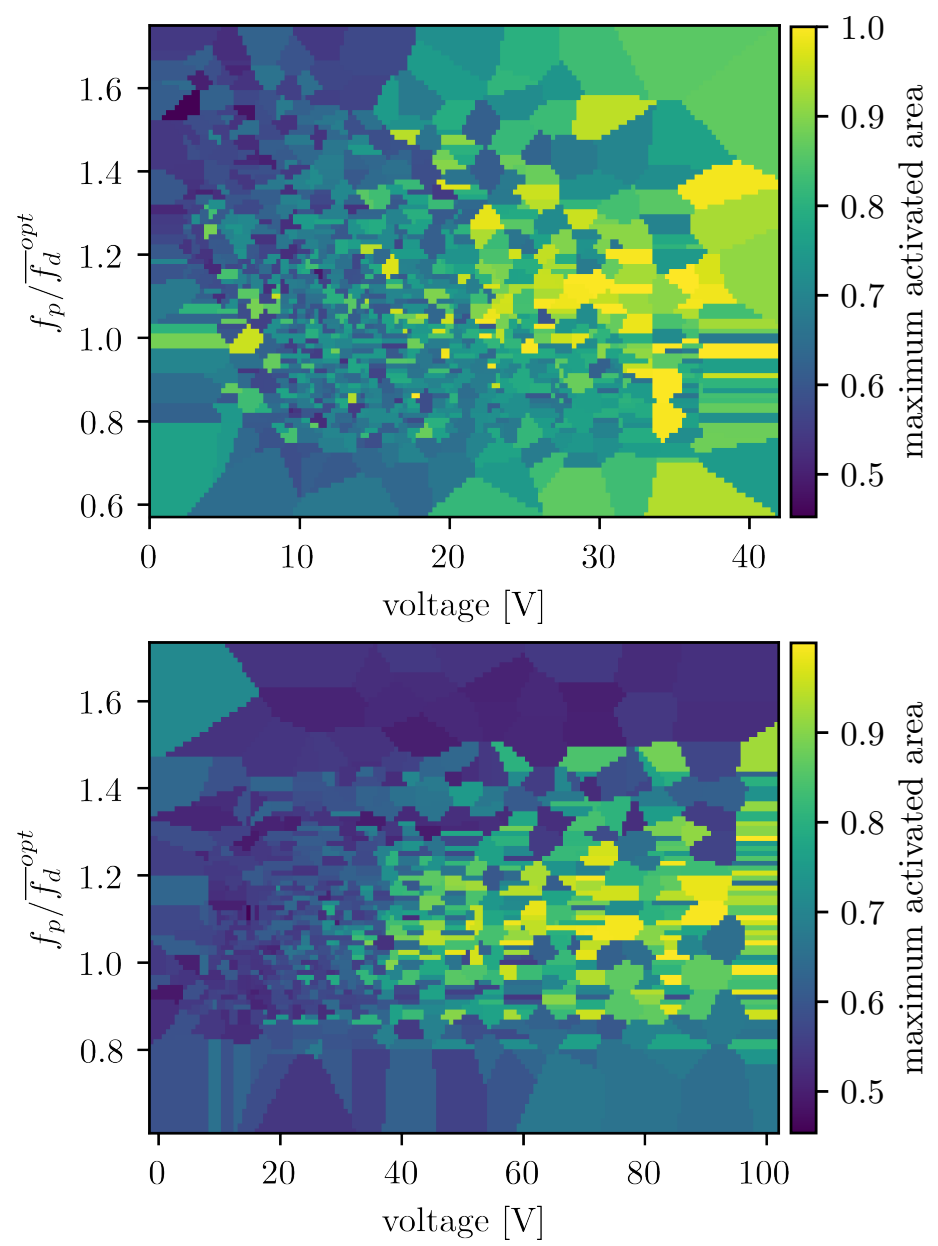

Figure A.4: Maximum activated area fraction caused by periodic pulses during VF. The diagram is analogous to the corresponding diagrams in fig. 4.7. However, here nearest neighbor interpolation was used in order to illustrate that close by parameter values can lead to very different maximum activated area fractions. This is the reason for the averaging that was applied in fig. 4.7. The upper (lower) diagram depicts data from experiments with rabbit (pig) hearts. Please see the caption of fig. 4.7 for further details. 

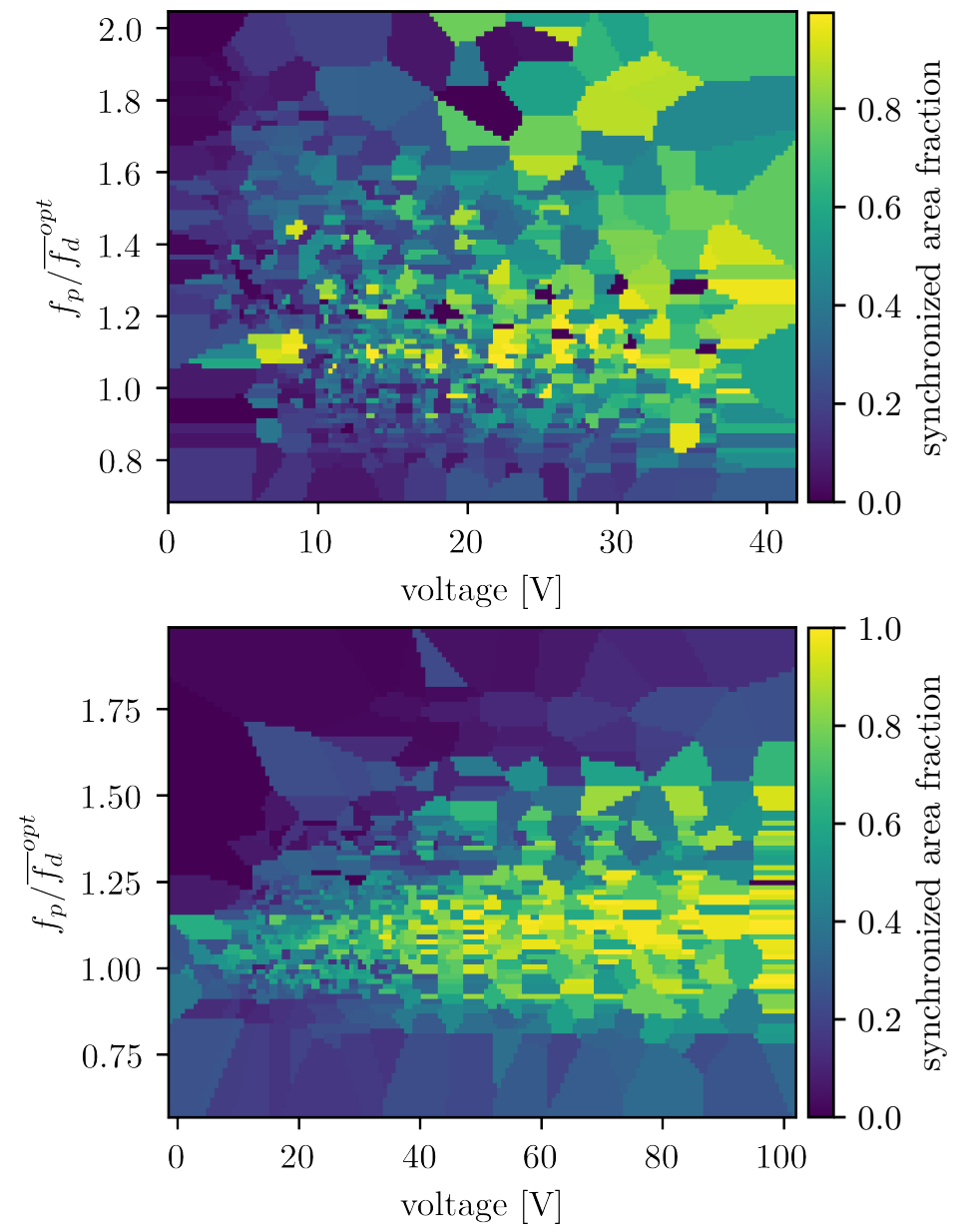

Figure A.5: Synchronized area fraction caused by periodic pulses during VF. The diagram is analogous to the corresponding diagrams in fig. 4.8. However, here nearest neighbor interpolation was used in order to illustrate that close by parameter values can lead to very different synchronized area fractions. This is the reason for the averaging that was applied in fig. 4.8. The upper (lower) diagram depicts data from experiments with rabbit (pig) hearts. Please see the caption of fig. 4.8 for further details. 


\section{A.6 Tables}

\begin{tabular}{lrrrrrr}
\hline & & $T_{p}[\mathrm{~s}]$ & $N_{p}$ & $T_{\text {gap }}[\mathrm{s}]$ & $N_{B}$ & $N$ \\
date & $U[\mathrm{~V}]$ & & & & & \\
\hline $2016-09-21$ & {$[4,35]$} & 0.09 & 80 & 4 & 15 & 1 \\
$2016-09-22$ & {$[4,35]$} & 0.1 & 80 & 4 & 15 & 1 \\
$2016-11-16$ & {$[2,30]$} & {$[0.1,0.15]$} & {$[35,41]$} & 4 & 15 & 14 \\
$2016-11-21$ & {$[9,37]$} & {$[0.11,0.13]$} & {$[31,39]$} & 4 & 10 & 6 \\
$2016-12-16$ & {$[9,37]$} & {$[0.07,0.12]$} & {$[35,54]$} & 5 & 10 & 10 \\
$2017-01-18$ & {$[18,18]$} & {$[0.08,0.09]$} & 40 & 4 & 5 & 2 \\
& {$[9,36]$} & {$[0.09,0.11]$} & 40 & 4 & 10 & 3 \\
& {$[9,37]$} & 0.1 & 40 & 4 & 10 & 2 \\
$2017-01-19$ & {$[9,37]$} & {$[0.07,0.11]$} & 44 & 4 & 10 & 5 \\
$2017-01-31$ & {$[18,18]$} & 0.1 & 40 & 4 & 10 & 2 \\
& {$[9,36]$} & {$[0.1,0.12]$} & 40 & 4 & 10 & 4 \\
$2017-02-02$ & {$[4,10]$} & 0.07 & 60 & 4 & 5 & 1 \\
& {$[4,15]$} & 0.06 & 80 & 4 & 7 & 1 \\
& {$[9,36]$} & {$[0.08,0.1]$} & 40 & 4 & 10 & 5 \\
$2017-03-13$ & {$[9,36]$} & {$[0.11,0.13]$} & 40 & 4 & 10 & 8 \\
$2017-03-15$ & {$[9,36]$} & {$[0.09,0.11]$} & 40 & 4 & 10 & 5 \\
$2017-03-17$ & {$[4,36]$} & {$[0.09,0.16]$} & 40 & 4 & 10 & 13 \\
$2017-04-20$ & {$[9,36]$} & {$[0.08,0.11]$} & 40 & 8 & 10 & 7 \\
$2017-04-28$ & {$[7,36]$} & {$[0.11,0.13]$} & 40 & 4 & 13 & 5 \\
$2017-05-03$ & {$[9,37]$} & {$[0.04,0.1]$} & 40 & 8 & 10 & 8 \\
$2017-05-08$ & {$[10,40]$} & 0.09 & 40 & 7 & 10 & 1 \\
& {$[5,40]$} & {$[0.06,0.11]$} & 40 & 7 & 10 & 7 \\
$2017-10-17$ & {$[15,35]$} & {$[0.04,0.09]$} & 30 & 8 & 6 & 7 \\
$2017-11-02$ & {$[18,35]$} & 0.05 & {$[40,60]$} & 10 & 7 & 2 \\
$2018-03-28$ & {$[10,40]$} & {$[0.07,0.14]$} & 40 & 10 & 10 & 8 \\
& {$[5,50]$} & 0.2 & 40 & 10 & 20 & 1 \\
$2018-04-05$ & {$[10,50]$} & {$[0.1,0.14]$} & 40 & 10 & 10 & 7 \\
$2018-05-07$ & {$[10,29]$} & {$[0.09,0.14]$} & 40 & 10 & 7 & 4 \\
& {$[10,50]$} & {$[0.1,0.16]$} & 40 & 10 & 10 & 13 \\
\hline & & & & & &
\end{tabular}

Table A.1: Further information on the parameters of Synchronization Maps in experiments with rabbit hearts. For each experiment (identified by the date) and each used range of voltages the following parameters are displayed: the pacing period $T_{p}$ rounded to one hundredth of a second, the number of pulses per block $N_{p}$, the time gap between blocks $T_{\text {gap }}$ rounded to full seconds, the number of blocks $N_{B}$ and the number of recorded Synchronization Maps $N$. If multiple values of a parameter were used, the range of values is denoted instead of a single voltage. 


\begin{tabular}{lrrrrrr}
\hline & & $T_{p}[\mathrm{~s}]$ & $N_{p}$ & $T_{\text {gap }}[\mathrm{s}]$ & $N_{B}$ & $N$ \\
date & $U[\mathrm{~V}]$ & & & & & \\
\hline $2016-10-11$ & {$[10,90]$} & 0.09 & 41 & 10 & 15 & 1 \\
& {$[30,90]$} & {$[0.09,0.11]$} & 41 & 5 & 10 & 6 \\
$2017-01-25$ & {$[0,8]$} & 0.15 & 27 & 4 & 10 & 1 \\
& {$[10,36]$} & {$[0.12,0.16]$} & {$[25,33]$} & 4 & {$[10,15]$} & 13 \\
& {$[18,70]$} & {$[0.16,0.18]$} & {$[22,25]$} & 4 & 10 & 5 \\
& {$[24,36]$} & 0.15 & 27 & 4 & 5 & 1 \\
& {$[36,100]$} & {$[0.13,0.15]$} & {$[26,31]$} & 4 & 6 & 2 \\
$2017-04-26$ & {$[50,100]$} & {$[0.14,0.23]$} & 40 & 4 & 10 & 1 \\
$2017-08-23$ & {$[6,100]$} & {$[0.13,0.18]$} & 40 & 10 & 15 & 4 \\
$2017-08-24$ & {$[6,100]$} & {$[0.1,0.12]$} & 40 & 10 & 15 & 2 \\
$2017-10-19$ & {$[24,100]$} & {$[0.08,0.12]$} & 30 & 10 & 8 & 6 \\
& {$[6,100]$} & {$[0.09,0.12]$} & 30 & 10 & 15 & 4 \\
$2017-10-25$ & {$[30,100]$} & {$[0.11,0.14]$} & 30 & 10 & 10 & 7 \\
$2018-02-13$ & {$[20,100]$} & {$[0.12,0.17]$} & 40 & 10 & 10 & 6 \\
& {$[50,50]$} & 0.14 & 40 & 10 & 15 & 1 \\
$2018-02-14$ & {$[20,100]$} & {$[0.11,0.14]$} & 40 & 10 & 10 & 7 \\
$2018-02-15$ & {$[20,100]$} & {$[0.14,0.17]$} & 40 & 10 & 10 & 6 \\
$2018-02-27$ & {$[20,100]$} & {$[0.12,0.14]$} & 40 & 10 & 10 & 9 \\
$2018-02-28$ & {$[20,100]$} & {$[0.08,0.11]$} & 40 & 10 & 10 & 4 \\
$2018-05-03$ & {$[20,100]$} & {$[0.08,0.11]$} & 40 & 10 & 10 & 4 \\
$2018-05-23$ & {$[20,100]$} & {$[0.12,0.21]$} & 40 & 10 & 10 & 12 \\
\hline
\end{tabular}

Table A.2: This table is identical to table A.1 but for recordings from pig hearts. Further information on the parameters of Synchronization Maps are provided. For each experiment (identified by the date) and each used range of voltages the following parameters are displayed: the pacing period $T_{p}$ rounded to one hundredth of a second, the number of pulses per block $N_{p}$, the time gap between blocks $T_{\text {gap }}$ rounded to full seconds, the number of blocks $N_{B}$ and the number of recorded Synchronization Maps $N$. If multiple values of a parameter were used, the range of values is denoted instead of a single voltage. 


\begin{tabular}{|c|c|c|c|c|c|c|c|c|c|c|}
\hline date & $U_{s}[\mathrm{~V}]$ & $P_{s}[\mathrm{~ms}]$ & bip. s. & $N_{s}$ & $r_{T}$ & $U_{u}[\mathrm{~V}]$ & $P_{u}[\mathrm{~ms}]$ & bip. u. & $\tau[\mathrm{s}]$ & $N$ \\
\hline 2016-08-17 & 20 & 6.0 & False & 10 & 0.9 & {$[39,47]$} & 8.0 & True & {$[0.05,0.09]$} & 12 \\
\hline 2016-08-22 & 20 & 6.0 & False & 10 & 0.9 & 75 & 6.0 & False & {$[0.07,0.12]$} & 7 \\
\hline 2016-08-23 & {$[20,35]$} & 6.0 & False & 10 & 0.9 & {$[75,82]$} & 6.0 & False & {$[0.07,0.12]$} & 90 \\
\hline 2016-09-12 & {$[25,30]$} & 6.0 & False & 10 & 0.9 & {$[51,68]$} & 8.0 & True & {$[0.07,0.11]$} & 67 \\
\hline 2016-11-21 & 30 & 5.0 & False & 25 & {$[0.95,1.05]$} & {$[51,68]$} & 7.0 & True & {$[0.08,0.1]$} & 37 \\
\hline 2016-11-25 & 20 & 5.0 & False & 25 & {$[0.95,1.05]$} & {$[62,68]$} & 7.0 & True & {$[0.08,0.13]$} & 13 \\
\hline $2016-12-16$ & 26 & 5.0 & False & 25 & {$[0.95,1.05]$} & {$[51,68]$} & 7.0 & True & {$[0.07,0.11]$} & 49 \\
\hline 2017-01-18 & {$[20,30]$} & 5.0 & False & 25 & {$[0.95,1.05]$} & {$[39,51]$} & 7.0 & True & {$[0.09,0.12]$} & 44 \\
\hline 2017-01-19 & 30 & 5.0 & False & 25 & {$[0.95,1.05]$} & 62 & 7.0 & True & {$[0.09,0.12]$} & 20 \\
\hline 2017-01-31 & 30 & 5.0 & False & 25 & {$[0.95,1.05]$} & {$[51,62]$} & 7.0 & True & {$[0.07,0.09]$} & 41 \\
\hline 2017-02-02 & 30 & 5.0 & False & 25 & {$[0.95,1.05]$} & {$[62,75]$} & 7.0 & True & {$[0.08,0.12]$} & 28 \\
\hline 2017-03-15 & 20 & 5.0 & False & 25 & {$[0.95,1.15]$} & {$[42,68]$} & 7.0 & True & {$[0.08,0.12]$} & 71 \\
\hline 2017-03-17 & {$[20,25]$} & 5.0 & False & 25 & {$[0.85,1.15]$} & {$[35,42]$} & 7.0 & True & {$[0.08,0.12]$} & 22 \\
\hline 2017-04-20 & 23 & 5.0 & False & 25 & {$[0.95,1.15]$} & {$[47,62]$} & 7.0 & True & 0.1 & 24 \\
\hline 2017-04-28 & 30 & 5.0 & False & 25 & {$[0.95,1.15]$} & {$[47,62]$} & 7.0 & True & 0.12 & 21 \\
\hline 2017-05-03 & 26 & 5.0 & False & 25 & {$[0.95,1.15]$} & {$[51,75]$} & 7.0 & True & 0.1 & 66 \\
\hline 2017-06-02 & {$[24,30]$} & 5.0 & False & 20 & {$[0.9,1.05]$} & {$[51,82]$} & 7.0 & True & {$[0.1,0.12]$} & 35 \\
\hline 2017-08-15 & 20 & 5.0 & False & 5 & 0.95 & {$[62,100]$} & 7.0 & True & 0.08 & 15 \\
\hline
\end{tabular}

Table A.3: Further information on the parameters of Syncrolation defibrillation attempts in experiments with rabbit hearts. For each experiment (identified by the date) the following parameters are displayed: the voltage $U_{s}$, the pulse width $P_{s}$, and the number of synchronizing pulses $N_{s}$, whether they are biphasic (bip. s.) and the period factor $r_{T}$ defining their frequency, the voltage $U_{u}$ and the pulse width $P_{u}$ of the unpinning pulse and whether they are biphasic (bip. u.) and the delay of the last pulse $\tau$ rounded to one hundredth of a second. The number of recorded Syncrolation attempts is denoted by $N$. If multiple values of a parameter were used, the range of values is denoted instead of a single voltage. 


\begin{tabular}{|c|c|c|c|c|c|c|c|c|c|c|}
\hline date & $U_{s}[\mathrm{~V}]$ & $P_{s}[\mathrm{~ms}]$ & bip. s. & $N_{s}$ & $r_{T}$ & $U_{u}[\mathrm{~V}]$ & $P_{u}[\mathrm{~ms}]$ & bip. u. & $\tau[\mathrm{s}]$ & $N$ \\
\hline 2017-01-27 & {$[80,100]$} & 5.0 & False & 25 & {$[0.95,1.05]$} & {$[910,1000]$} & 5.0 & True & {$[0.1,0.14]$} & 16 \\
\hline $2017-05-23$ & 50 & 5.0 & False & 20 & {$[0.85,1.15]$} & {$[750,1000]$} & 6.0 & True & {$[0.14,0.19]$} & 22 \\
\hline 2017-08-24 & 50 & 5.0 & False & 20 & 0.95 & {$[470,1000]$} & 7.0 & True & {$[0.1,0.14]$} & 37 \\
\hline 2017-10-19 & {$[51,100]$} & 5.0 & False & 20 & {$[0.95,1.1]$} & {$[620,1000]$} & 7.0 & True & {$[0.08,0.12]$} & 30 \\
\hline $2017-10-25$ & {$[62,70]$} & 5.0 & False & {$[20,30]$} & 0.9 & {$[680,1000]$} & 7.0 & True & 0.1 & 10 \\
\hline 2018-02-13 & 42 & 5.0 & False & 25 & 0.91 & {$[750,1000]$} & 7.0 & True & 0.1 & 9 \\
\hline 2018-02-14 & 60 & 5.0 & False & 25 & 0.91 & {$[510,820]$} & 7.0 & True & 0.1 & 10 \\
\hline 2018-02-15 & 60 & 5.0 & False & 25 & 0.91 & {$[420,510]$} & 7.0 & True & 0.1 & 3 \\
\hline
\end{tabular}

Table A.4: Further information on the parameters of Syncrolation defibrillation attempts in experiments with pig hearts. For each experiment (identified by the date) the following parameters are displayed: the voltage $U_{s}$, the pulse width $P_{s}$, and the number of synchronizing pulses $N_{s}$, whether they are biphasic (bip. s.) and the period factor $r_{T}$ defining their frequency, the voltage $U_{u}$ and the pulse width $P_{u}$ of the unpinning pulse and whether they are biphasic (bip. u.) and the delay of the last pulse $\tau$ rounded to one hundredth of a second. The number of recorded Syncrolation attempts is denoted by $N$. If multiple values of a parameter were used, the range of values is denoted instead of a single voltage. 


\begin{tabular}{llll}
\hline & $E_{50}^{1 B P}[\mathrm{~J}]$ & $E_{50}^{5 B P}[\mathrm{~J}]$ & $E_{50}^{\text {Unin }}[\mathrm{J}]$ \\
date & & & \\
\hline $2016-11-21$ & nan \pm nan & $0.4 \pm 0.1$ & $1.2 \pm 0.1$ \\
$2016-12-16$ & nan \pm nan & $0.8 \pm 0.1$ & $1.2 \pm 0.1$ \\
$2017-01-18$ & $0.8 \pm 0.1$ & $0.6 \pm 0.1$ & $0.7 \pm 0.1$ \\
$2017-01-19$ & $1.9 \pm 0.6$ & $1.2 \pm 0.4$ & $1.3 \pm 0.2$ \\
$2017-01-31$ & $1.2 \pm 0.1$ & $0.8 \pm 0.1$ & $1.2 \pm 0.1$ \\
$2017-02-02$ & $1.6 \pm 0.3$ & $1.2 \pm 0.2$ & $1.7 \pm 0.2$ \\
$2017-04-28$ & $1.3 \pm 0.4$ & $1.1 \pm 0.3$ & $1.1 \pm 0.2$ \\
$2017-05-03$ & $1.8 \pm 0.3$ & $1.0 \pm 0.2$ & $1.5 \pm 0.2$ \\
$2017-06-02$ & $2.7 \pm 0.5$ & $1.6 \pm 0.3$ & $1.8 \pm 0.3$ \\
\hline
\end{tabular}

Table A.5: The characteristic energies of a single shock, five underdrive pulses, and Syncrolation are shown for nine experiments with rabbit hearts.

\begin{tabular}{llllr}
\hline & & & & $N$ \\
date & $N_{s}$ & $U_{s}[\mathrm{~V}]$ & $r_{T}[\mathrm{~s}]$ & \\
\hline $2016-11-21$ & 25 & 30.0 & 0.95 & 18 \\
& & & 1.05 & 19 \\
$2016-12-16$ & 25 & 26.0 & 0.95 & 24 \\
& & & 1.05 & 25 \\
$2017-01-18$ & 25 & 30.0 & 0.95 & 16 \\
& & & 1.05 & 17 \\
$2017-01-19$ & 25 & 30.0 & 0.95 & 9 \\
& & & 1.05 & 11 \\
$2017-01-31$ & 25 & 30.0 & 0.95 & 21 \\
& & & 1.05 & 20 \\
$2017-02-02$ & 25 & 30.0 & 0.95 & 14 \\
& & & 1.05 & 14 \\
$2017-04-28$ & 25 & 30.0 & 0.95 & 5 \\
& & & 1.05 & 6 \\
$2017-05-03$ & 25 & 26.0 & 0.95 & 17 \\
& & & 1.05 & 17 \\
$2017-06-02$ & 20 & 30.0 & 0.95 & 9 \\
& & & 1.05 & 9 \\
\hline
\end{tabular}

Table A.6: The experiment dates, pulse settings and number of defibrillation attempts of the rabbit dataset CRSyncr is shown. Each row corresponds to one pulse setting and the last column shows the corresponding number of defibrillation attempts. 


\begin{tabular}{l|r} 
Ingredient & Concentration $[\mathrm{mM}]$ \\
\hline D-Glucose & 11.1 \\
$\mathrm{MgSO}_{4}$ & 1.2 \\
$\mathrm{KH}_{2} \mathrm{PO}_{4}$ & 1.2 \\
$\mathrm{KCl}$ & 4.7 \\
$\mathrm{NaCl}$ & 118.1 \\
$\mathrm{CaCl}_{2}$ & 3.4 \\
$\mathrm{NaHCO}_{3}$ & 25.0
\end{tabular}

Table A.7: Composition of the used Krebs-Henseleit solution.

\begin{tabular}{l|r} 
Ingredient & Concentration $[\mathrm{mM}]$ \\
\hline $\mathrm{D}-\mathrm{Glucose}$ & 12.0 \\
$\mathrm{NaH}_{2} \mathrm{PO}_{4}\left(\mathrm{H}_{2} \mathrm{O}\right)$ & 1.2 \\
$\mathrm{KCl}$ & 4.0 \\
$\mathrm{MgCl}$ & $\left(6 \mathrm{H}_{2} \mathrm{O}\right)$ \\
$\mathrm{NaCl}$ & 0.6 \\
$\mathrm{CaCl}_{2}$ & 130.0 \\
$\mathrm{NaHCO}_{3}$ & 2.2 \\
& 24.2
\end{tabular}

Table A.8: Composition of the used Tyrode solution. 
A Appendix

\section{A.7 Supplementary Material to Manuscript}

The following pages contain the supplementary material to the manuscript contained in chapter 2. 
Place holder for the supplementary material of

H. tom Wörden, U. Parlitz, and S. Luther. Simultaneous unpinning of multiple vortices in two-dimensional excitable media. Physical Review E, E 99, 042216, 2019. DOI: 10.1103/PhysRevE.99.042216 